$$
\begin{gathered}
\text { Aus dem Fachbereich Medizin } \\
\text { der Johann Wolfgang Goethe-Universität } \\
\text { Frankfurt am Main }
\end{gathered}
$$

$$
\begin{gathered}
\text { betreut am } \\
\text { Zentrum der Chirurgie }
\end{gathered}
$$

Klinik für Unfall-, Hand- und Wiederherstellungschirurgie

Direktor Prof. Dr. Ingo Marzi

\title{
Welche Kompetenzen erwerben Studierende im Blockpraktikum Chirurgie - Curriculum Mapping mit dem NKLM
}

\author{
Dissertation \\ zur Erlangung des Doktorgrades der Medizin \\ des Fachbereichs Medizin \\ der Johann Wolfgang Goethe-Universität \\ Frankfurt am Main
}

vorgelegt von

Anna-Maria Lübbehüsen

aus Bad Homburg vor der Höhe

Frankfurt am Main, 2021 
Dekan:

Referentin:

Korreferent:

Tag der mündlichen Prüfung: $\quad$ 11.11.2021
Prof. Dr. Stefan Zeuzem

Prof. Dr. Miriam Rüsseler

Prof. Dr. Stefan Rüttermann 


\section{Inhaltsverzeichnis}

Inhaltsverzeichnis $\quad 4$

Abkürzungen $\quad 6$

$\begin{array}{ll}\text { Zusammenfassung } & 7\end{array}$

Summary 9

1 Einleitung 11

1.1 Allgemeine Überlegungen 11

1.2 ÄApprO: Ärztliche Ausbildung 12

1.3 Lernziele $\quad 14$

1.4 Kompetenzorientiertes Lernen 15

$\begin{array}{ll}1.5 \text { NKLM und CanMEDS-Rollen } & 19\end{array}$

1.6 Mapping 26

1.7 Chirurgisches Curriculum Frankfurt 29

2. Fragestellung 32

3. Material und Methoden 33

3.1 Studiendesign 33

3.2 Studienteilnehmende 33

3.3 Studienprotokoll 33

3.3.1 Studienvorbereitung 33

3.3.2 Bearbeitung des Lernzielbogens $\quad 35$

3.3.3 Studienzeitraum 35

3.3.4 Rekrutierung der Studienteilnehmenden 35

3.3.5 Lehrkrankenhäuser und Zuteilung 36

3.3.6 Fachrichtungen $\quad 37$

3.3.7 Inhalte des Blockpraktikums Chirurgie 38

3.4 Statistische Auswertung 38

4. Ergebnisse 40

4.1 Teilnehmer 40

4.2 Fachrichtungen $\quad 40$

4.3 Lehrkrankenhäuser + Uniklinikum 41

4.4 Lernziele $\quad 42$ 
4.4.1 gesamt 42

4.4.2 Abschnitte $1-3$ und Kapitel 43

4.4.3 männlich/weiblich $\quad 49$

4.4.4 Fachrichtungen gesamt $\quad 53$

4.4.5 Uni/Lehrkrankenhäuser gesamt $\quad 62$

4.5 Benötigte Ressourcen 70

$\begin{array}{ll}\text { 5. Diskussion } & 71\end{array}$

5.1 Allgemeines $\quad 71$

5.1.1 Arbeitsaufwand Mapping $\quad 71$

5.1.2 CanMEDS $\quad 72$

5.1.2.1 Klinische Relevanz, Rollenverständnis 72

5.1.2.2 Anwendung in Prüfungen $\quad 74$

5.1.3 Wichtigkeit klinisch-praktischer Ausbildung 75

5.2 Unterschied Geschlechter 76

5.2.1 Selbstwirksamkeit Männer und Frauen + Effekt auf Karriere 76

5.2.2 Überschätzer/Unterschätzer 78

$\begin{array}{ll}5.3 \text { Fachrichtungen } & 80\end{array}$

5.3.1 Chirurgie und praktische Fertigkeiten 80

5.4 (Lehr-) Krankenhäuser 81

5.4.1 Akademische Lehrkrankenhäuser, Aufwendung für BP 81

5.4.2 Lehre im klinischen Alltag 82

6. Schlussfolgerungen $\quad 85$

7. Literaturverzeichnis 86

$\begin{array}{ll}\text { 8. Abbildungsverzeichnis } & 97\end{array}$

9. Tabellenverzeichnis 99

10. Anhang 100

11. Danksagung 104

12. Curriculum vitae 105

13. Veröffentlichungen und Vorträge 106

14. Schriftliche Erklärung 107 


\section{Abkürzungen}

ÄApprO Ärztliche Approbationsordnung

BP Blockpraktikum

LZ Lernziel

LZK Lernzielkatalog

Max. Maximum

MCQ Multiple Choice Question

Min. Minimum

MW Mittelwert

NKLC Nationaler Kompetenzbasierter Lernzielkatalog Chirurgie

NKLM Nationaler Kompetenzbasierter Lernzielkatalog Medizin

OSCE Objective structured clinical examination

PJ Praktisches Jahr

SCLO Swiss Catalogue of Learning Objectives for Undergraduate Medical Training

SD Standardabweichung

\pm SD Standardabweichung Positiv/Negativ

TPF Training Praktischer Fertigkeiten

Hinweis: Ausschließlich aus Gründen der besseren Lesbarkeit wird im Text nur das generische Maskulinum verwendet. Gemeint ist stets sowohl die weibliche als auch die männliche Form. 


\section{Zusammenfassung}

\section{Hintergrund}

Die Verankerung der Kompetenzorientierung und die Betonung der praktischklinischen Ausbildung im Rahmen des Medizinstudiums sind zentrale Punkte in den Neuerungen der Ärztlichen Approbationsordnung. Mit der Entwicklung des Nationalen Kompetenzbasierten Lernzielkatalogs Medizin (NKLM) ist ein Rahmenwerk erstellt und verabschiedet worden, das die Inhalte des gesamten Medizinstudiums in Deutschland abbilden und eine Implementierung kompetenzorientierter Lernziele an den Fakultäten forcieren soll. Um diesem Ziel gerecht zu werden, müssen bereits an den Fakultäten vorhandene Lehrveranstaltungen mit dem NKLM abgeglichen und im Rahmen eines Curriculum Mappings kartiert werden. Ziel der vorliegenden Arbeit ist daher die Kartierung der im Frankfurter Blockpraktikum Chirurgie erlernten Kompetenzen im Sinne eines Curriculum Mappings in Anlehnung an die im NKLM formulierten Lernziele. Zudem wurde folgenden Fragestellungen nachgegangen: Welcher Umfang kompetenzorientierter Lernziele kann Studierenden in einem zweiwöchigen Praktikum Chirurgie vermittelt werden? Wie ist die Vermittlung der einzelnen Kapitel des NKLM im Blockpraktikum Chirurgie gewichtet? Gibt es Unterschiede der erreichten Lernziele in Abhängigkeit des Geschlechts der Studierenden, der besuchten Fachrichtung bzw. des besuchten Lehrkrankenhauses?

\section{Material und Methoden}

Im Rahmen der vorliegenden Arbeit wurden Medizinstudierende im zweiten bzw. dritten klinischen Semester unmittelbar nach Abschluss ihres Blockpraktikums Chirurgie gebeten, unter Nutzung eines Online-Fragebogens anzugeben, welche der im NKLM formulierten Lernziele sie im zweiwöchigen Blockpraktikum Chirurgie gelernt haben. Somit konnte für jedes Kapitel dargestellt werden, zu welchem prozentualen Anteil die Lernziele dieses Kapitels erreicht worden sind. Zudem wurden die soziodemographischen Daten der Studierenden, die Fachrichtung des 
Blockpraktikums und das Lehrkrankenhaus erfasst. Die statistische Auswertung erfolgte mit dem Wilcoxon-Mann-Whitney Test und dem Kruskal-Wallis Test.

\section{Ergebnisse}

Insgesamt nahmen 81 Studierenden (28 Männer, 53 Frauen) aus dem 2. bzw 3. klinischen Semester an der Studie teil. Insgesamt wurden im zweiwöchigen Blockpraktikum Chirurgie 8,78 \pm 5,10\% (Min. 1,01\%; Max. 29,84\%) aller Lernziele von den Studierenden erreicht. Hierbei wurden anteilig die meisten Lernziele in den Kapiteln 5-11 (Abschnitt 1 „Ärztliche Rollen“) mit 29,92 — 15,22\% (Min. 0,00\%; Max. 63,10\%) vermittelt. Aus Abschnitt 2 (,Medizinisches Wissen, klinische Fähigkeiten und professionelle Haltungen") wurden vor allem die Lernziele der Kapitel 14b „Klinisch-praktische Fertigkeiten“ (15,49 \pm 7,78\% (Min. 0,00\%; Max. 41,30\%) und 14c „Ärztliche Gesprächsführung“ (22,98 \pm 16,47\% (Min. 0,00\%; Max. 70,69\%) von den Studierenden erreicht. Männer geben durchschnittlich an, mehr Lernziele erreicht zu haben als Frauen $(9,84 \%$ vs. $8,22 \%$; $p=0.104731)$. Weiterhin haben Studierende, die ihr Praktikum in einem Lehrkrankenhaus mit weniger als 100 chirurgischen Bettenplätzen (10,60 \$6,75\%; Min. 2,33\%; Max. 29,84\%) oder in einer Rotation (9,95 \pm 6,67\%; Min. 1,90\%; Max. 29,84\%) durch mehrere Fachrichtungen absolvierten, angegeben mehr Lernziele erreicht zu haben als andere Studierende insgesamt.

\section{Schlussfolgerung}

Das zweiwöchige Blockpraktikum Chirurgie in Frankfurt kann den Studierenden (im Hinblick auf die Gesamtdauer des Medizinstudiums) einen großen Anteil der im NKLM formulierten Lernziele vermitteln. Vor allem die Lernziele der „Ärztlichen Rollen“ und der „klinisch-praktischen Fertigkeiten“ werden erlernt. Die Vermittlung gelingt besonders umfangreich in kleineren Lehrkrankenhäusern. Trotzdem bietet das Blockpraktikum Chirurgie den Teilnehmer nur einen kleinen Einblick in den Fachbereich Chirurgie. Für die Vermittlung von spezifischen chirurgischen Fähigkeiten, Prinzipen chirurgischer Diagnostik und Therapie, sowie Aspekte der „Patientenzentrierten Gesundheitsversorgung“ sind andere Formate notwendig. 


\section{Summary}

\section{Background}

Anchoring competences and the accentuation of practical-clinical education are central aspects of reforming the Licensing Regulations for Doctors (Approbationsordnung). The development of the German National Competencebased Learning Objectives Catalogue for Undergraduate Medical Education (NKLM) has created a framework that reflects the content of the German national medical curriculum and forces the implementation of competency based learning objectives. To fulfill these goals, faculties have to compare their existing teaching sessions with the NKLM and map them within the context of curriculum mapping.

Aim of this study is to map competences learned during the surgical internship at the medical faculty of Goethe University Frankfurt/Main within the scope of Curriculum mapping on the basis of NKLM learning objectives.

Furthermore the following questions were adressed:

Which number of competency-based learning objectives can be conveyed to students during the surgical internship?

How are the chapters and the according learning objectives of the NKLM weighted? Are there differences in learned objectives according to gender, surgical specialization and teaching hospital?

\section{Methods}

For this study third year medical students at the medical faculty of Goethe University Frankfurt/Main were asked to declare what they learned during the surgical internship with the use of an online-based questionnaire. Using this method on each chapter of the NKLM, we were able to show which percentage of the learning objectives had been achieved. Furthermore socio-demographic data was collected as well as data on surgical department and teaching hospital. Statistical analysis was carried out with MS Excel an bias using Wilcoxon-Mann-Whitney-U- and Kruskal-Wallis-Tests. 


\section{Results}

81 third year medical students ( 28 men and 53 women) from the medical faculty of Goethe University Frankfurt/Main were included in this study. In general $8,78 \pm 5,10 \%$ (Min. $=1,01 \%$; Max. $=29,84 \%)$ of all learning objectives of the NKLM were achieved after participating in the two-weeks surgical internship. The highest result $(29,92 \pm 15,22 \%$; Min. $=0,00 \%$; Max. $=63,10 \%)$ was achieved in chapter $5-11$ part 1 „Professional roles for medical doctor").

In part 2 of the NKLM („Medical knowledge, clinical skills and professionell manner“) students could especially accomplish learning objectives of chapters $14 \mathrm{~b}$ „practicalmedical skills" (15,49 $\pm 7,78 \%$; Min. $=0,00 \%$; Max. $=41,30 \%)$ and $14 \mathrm{c}$ "doctor-patient interaction" (22,98 $\pm 16,47 \%$; Min. $=0,00 \%$; Max. $=70,69 \%)$. On average, male

participants report reaching more objectives than women $(9,84 \%$ vs. $8,22 \%$; $p=$ $0.104731)$. Furthermore students who undergo their surgical-practical course at a teaching hospital with under 100 surgical beds (10,60 $\pm 6,75 \%$; Min. $=2,33 \%$; Max. $=$ $29,84 \%)$ or joined a variety of surgical departments $(9,95 \pm 6,67 \%$; Min. $=1,90 \%$; Max. $=29,84 \%$ ), report having achieved more learning objectives than those in other hospitals or in a single department.

\section{Conclusion}

This study shows that the two-weeks surgical internship of the medical faculty of Goethe University Frankfurt/Main can convey a relevant amount of learning objectives as defined in the NKLM to the participants. There is evidence to suggest that especially learning objectives concerning „Professional roles for medical doctors" and clinical-practical skills were reached by the students. Teaching is particularily successful in smaller teaching hospitals. Furthermore, our data allows us to conclude that in order to teach students specialized surgical skills, principles of surgical diagnosis and therapy, and aspects of part 3 of the NKLM („Guiding symptoms" and "Diseases"), other teaching formats are needed. 


\section{Einleitung}

\subsection{Allgemeine Überlegungen}

Das Medizinstudium zählt mit einer Dauer von 6 Jahren zu den umfangreichsten Studiengängen. ${ }^{1}$ Es ist zudem seit Jahren auch einer der begehrtesten Studiengänge Deutschlands, wenn man die Zahl der Bewerber mit den Zahlen der Studienplätze vergleicht. ${ }^{2}$ Auch das Berufsbild des Arztes nimmt eine Schlüsselfunktion in der Gesellschaft und im Gesundheitswesen ein. In der (Muster) Berufsordnung für die in Deutschland tätigen Ärztinnen und Ärzte (MBO-Ä) werden die zentralen Aufgaben in $\S 1$ wie folgt beschrieben: „(1) Ärztinnen und Ärzte dienen der Gesundheit des einzelnen Menschen und der Bevölkerung. Der ärztliche Beruf ist kein Gewerbe. Er ist seiner Natur nach ein freier Beruf.(2) Aufgabe der Ärztinnen und Ärzte ist es, das Leben zu erhalten, die Gesundheit zu schützen und wiederherzustellen, Leiden zu lindern, Sterbenden Beistand zu leisten und an der Erhaltung der natürlichen Lebensgrundlagen im Hinblick auf ihre Bedeutung für die Gesundheit der Menschen mitzuwirken." Weiter heißt es in § 2: „(2) Ärztinnen und Ärzte haben ihren Beruf gewissenhaft auszuüben und dem innen bei ihrer Berufsausübung entgegengebrachten Vertrauen zu entsprechen. Sie haben dabei inr ärztliches Handeln am Wohl der Patientinnen und Patienten auszurichten." Daraus ergeben sich erhebliche Anforderungen an das Medizinstudium auf mehreren Dimensionen: Das Medizinstudium soll demnach die Absolventen befähigen, Kompetenzen auf den unterschiedlichsten Ebenen zu erlernen, die der hohen Verantwortung des ärztlichen Berufs gerecht werden. Dazu zählen unter anderem Kenntnisse im Bereich der wissenschaftlichen Analyse, evidenzbasierte Interpretation von Zahlen und Fakten, der Anwendung von diagnostischen und therapeutischen Prinzipien, der Patientenkommunikation, der Teamarbeit, der Physiologie des menschlichen Körpers und der Pathophysiologie von Erkrankungen. ${ }^{3}$ Auch die Vertretungen der Studierenden greifen die wachsende Komplexität des Anforderungsprofils auf und bilden daraus Forderungen an die ärztliche Ausbildung. So schreibt die Bundesvertretung der Medizinstudierenden in Deutschland (bvmd) in ihrem Positionspapier zur Zukunft und Weiterentwicklung des 
Medizinstudiums": „Die bvmd spricht sich dafür aus, die Struktur des Medizinstudiums bundeseinheitlich $\mathrm{zu}$ regeln. Außerdem muss sich das Medizinstudium immer an den Bedürfnissen derjenigen Gesellschaft, in der die Medizinstudierenden ausgebildet werden, orientieren, um eine adäquate Vorbereitung zukünftiger Ärzte auf den ärztlichen Alltag zu gewährleisten.“

\subsection{Die ärztliche Approbationsordnung}

Die rechtliche Grundlage, die Inhalte des Studiums und den zeitlichen Rahmen legt die Approbationsordnung für Ärzte ${ }^{1}$ fest. Sie wird auf Basis der Bundesärzteordnung (BÄO) vom Bundesministerium für Gesundheit erlassen. In ihr ist festgelegt, dass die 6-jährige Ausbildung aus wissenschaftlich-theoretischen und klinischepraktischen Anteilen bestehen soll, sowie fakultäre und staatliche Prüfungsleistungen zu absolvieren sind. Die Vermittlung der Inhalte obliegt dabei den einzelnen Universitäten. Die Ziele der ärztlichen Ausbildung werden in der aktuellen Fassung der Approbationsordnung in $\S 1$ wie folgt formuliert: „Ziel der ärztlichen Ausbildung ist der wissenschaftlich und praktisch in der Medizin ausgebildete Arzt, der zur eigenverantwortlichen und selbständigen ärztlichen Berufsausübung, zur Weiterbildung und zu ständiger Fortbildung befähigt ist." "1 Dennoch zeigen Studien, dass sich häufig junge Assistenzärzte den Ansprüchen eben diese eigenverantwortlichen und selbstständigen Berufsausübung nicht gewachsen sehen. ${ }^{5}$ Die Inhalte des Medizinstudiums und deren Entwicklung waren daher in den letzten Jahrzehnten immer wieder Bestandteil der öffentlichen und politischen Diskussion, im Rahmen des Bologna Prozesses oder der umfassenden Reform der ärztlichen Approbationsordnung 2002 und sind es immer noch ${ }^{6-8}$. Bereits in den 1980er Jahren forderte der Wissenschaftsrat die medizinische Ausbildung hinsichtlich einer berufspraktischen Ausrichtung zu reformieren. ${ }^{9}$ Weitere Inhalte der bis heute geführten Diskussionen sind vor allem der Wandel des Arztbildes in der demographischen Entwicklung der Gesellschaft sowie eine stärkere Verankerung der Wissenschaftlichkeit im Studium und die gestiegene Notwendigkeit eines

vermehrten Praxisbezuges. Internationale Reformcurricula unter anderem aus Nordamerika, ${ }^{10}$ Großbritannien ${ }^{11}$, Schottland, ${ }^{12}$ den Niederlanden ${ }^{13}$ und der 
Schweiz $^{14}$ haben im weiteren Verlauf Einfluss auf die Entwicklung der Medizinstudiums in Deutschland genommen. Durch tiefgreifende Reformen soll ein Übergang geschaffen werden, der eine Wissensvermittlung anhand von ärztlichen Rollen und Kompetenzen etabliert. Als Grundgerüst gilt an dieser Stelle das nordamerikanische Konzept von 2005 zur Definition der Rolle des Arztes, das sogenannte "CanMEDS 2005 Framework". ${ }^{10}$ Auch der frühzeitige Praxis- und Patientenbezug, sowie die longitudinale und vertikale Integration grundlegender und klinischer Lehrinhalte gilt als wichtiger Bestandteil der Reformen. ${ }^{9}$ Mit der 1999 eingeführten Reformklausel zur Einführung von Modellstudiengängen, in der 2002 erlassenen umfassenden Reform der ÄApprO und in deren Überarbeitung 2013 werden diese Aspekte aufgegriffen und verankert. Neben geänderten Strukturvorgaben wird hier auch die Notwendigkeit einer didaktisch hochwertigen und fundierten Vermittlung von Lehrinhalten betont. ${ }^{9,15}$ Eine Umstrukturierung von prozessorientierter hin zu ergebnisorientierter Lehre soll somit weiter ermöglicht und vollzogen werden. ${ }^{16}$ Weiterhin wird so zunehmend Wert auf die Stärkung der Ausbildung in praktisch klinischer Kompetenz gelegt. Der am 31. März 2017 gemeinsam durch die Wissenschafts- und Gesundheitsressorts von Bund und Ländern verabschiedete Masterplan Medizinstudium 2020 knüpft an diese wesentlichen Reformimpulse an ${ }^{17}$ : Patienten- und Praxisbezug, Kompetenzorientierung und vertikale und horizontale Integration von Lehrinhalten in die medizinische Ausbildung sind wesentliche Inhalte. ${ }^{9}$ Die Unterrichtsinhalte des Medizinstudiums sollen dementsprechend künftig fächerübergreifendes Denken fördern und, soweit zweckmäßig, problemorientiert vermittelt werden. Die Vermittlung der naturwissenschaftlichen und theoretischen Grundlagen ist auf die medizinisch relevanten Ausbildungsinhalte zu konzentrieren und theoretisches und klinisches Wissen soll während der gesamten Ausbildung so weitgehend wie möglich miteinander verknüpft werden. ${ }^{17}$

Dennoch ist die ÄApprO in Bezug auf die formulierten Reformpläne einer wesentlichen Limitation unterworfen: Die erforderlichen Ausbildungsziele werden nicht explizit in Form von berufsrelevanten Rollen und zugehörigen Kompetenzen wiedergegeben. Die Curricula des Medizinstudiums werden durch die Vorgaben der 
ÄApprO lediglich rechtlich vorstrukturiert. Eine Spezifizierung zu Inhalt und Umfang beschreibt die Approbationsordnung nicht. Durch diese vage Formulierung ergibt sich ein großer Gestaltungsspielraum ${ }^{18}$ und die Ausgestaltung der Vorgaben der ÄApprO wird von den einzelnen medizinischen Fakultäten selbst vorgenommen. So existiert eine Vielzahl medizinischer Curricula an den einzelnen Fakultäten auf nationaler Ebene. ${ }^{19,20}$ Es ist anzunehmen, dass sich diese uneinheitliche Abbildung auch auf andere Fächer übertragen lässt.

\subsection{Lernziele}

Präzise formulierte Lernziele bieten die Grundlage einer didaktisch hochwertigen und zielführenden Lehre. Eine der meist zitierten Definition von Lernzielen formuliert der Pädagoge Robert F. Mager. Er definiert Lernziele als eine zweckmäßige Zielbeschreibung, mit der es gelingt, die Unterrichtsabsichten dem Leser mitzuteilen. ${ }^{21}$ So sind Lernziele die möglichst exakte Beschreibung des angestrebten Lernergebnisses. ${ }^{22}$ Die in der medizinischen Ausbildungsforschung verwendete Definition von Lernzielen ist analog hierzu: Lernziele sind eine breite Beschreibung dessen, was am Ende einer Lerneinheit erreicht und abgeprüft worden ist. Sie sind ein unmittelbarer Bestandteil von Curricula, Lehr- und Lernmethoden und Prüfungen. ${ }^{23}$

Um Studierende hierbei bestmöglich beim Lernen zu unterstützen, existiert das Konzept, Lernziele im Sinne einer Lernspirale zu formulieren. ${ }^{24}$ Die Strukturierung der Ausbildungsinhalte nach der Vorstellung einer Lernspirale ist bereits 1960 von Jerome Bruner beschrieben worden. ${ }^{25}$ Dabei sollen die einzelnen Themen und Lernziele immer wieder überdacht und rekapituliert werden, neu in Bezug gesetzt werden und Neues mit bereits Gelerntem verknüpft werden. So ergibt sich ein iteratives Konzept, das steigende Anforderungslevel und Kompetenzlevel kreiert. Die schrittweise Heranführung an höhere Anforderungslevel soll Überforderung vermeiden. ${ }^{24} \mathrm{Um}$ die Lernziele hinsichtlich Lerntiefe und Komplexität spezifizieren zu können, können diese beispielsweise entsprechend der Taxonomiestufen nach Bloom weiter ausformuliert werden. ${ }^{26}$ Lernziele bestimmen den Inhalt und die Organisation des Curriculums, die Lehrmethoden und die Prüfungsformate und 
geben einen Anhalt für die Evaluation von Curricula. Die Formulierung von Lernzielen muss so erfolgen, dass sie gut in Curricula, die ständig wachsen und sich wandeln, integriert werden können, um so Ausbildungs- und Prüfungsinhalten neue Herausforderungen anpassen zu können. ${ }^{17}$ Die Formulierung von Lernzielen sollte unmittelbar mit dem Prüfungskonzept in Verbindung: Bereits 1996 formulierte John Biggs das Prinzip des Constructive Alignment von im Sinne von "what you test is what they learn". ${ }^{27}$ Um die Studierenden zum Tiefenlernen und Kompetenzerwerb anzuregen, müssen im Sinne eines Constructive Alignments neben den Inhalten somit vor allem die Prüfungen an den erwünschten Lernergebnissen ausgerichtet sein: nur so kann sichergestellt werden, dass Studierende die angestrebten Kompetenzen erwerben ${ }^{28}$ und sich eine Lerngelegenheit für Studierende und Dozenten ergibt. ${ }^{29}$

Bundesweit sind daher bereits verschiedene Lernzielkataloge formuliert worden, die Lernziele für Medizinstudierende definieren. Hierbei reicht die Spanne von Lernzielkatalogen für einzelnen Fächer an einer einzigen Fakultät ${ }^{30}$ bis hin zum vom Institut für medizinische und pharmazeutische Prüfungsfragen (IMPP) formulierten Gegenstandskatalog für die bundeseinheitlichen Staatsexamina. Auch einzelne Fachgesellschaften haben in der Vergangenheit Lernzielkataloge für ihr Fach formuliert: Seit 2006 existiert beispielsweise ein Lernzielkatalog des Faches Unfallchirurgie und Orthopädie. ${ }^{31} 2015$ ist der darüber hinaus der bundesweit einheitliche Nationale Kompetenzorientierte Lernzielkatalog Medizin (NKLM) verabschiedet worden ${ }^{32}$, der im Weiteren genauer beschrieben wird. Auf Grundlage des NKLM sind weitere Kataloge einzelner Fachrichtungen, wie zum Beispiel der Psychosomatik ${ }^{33}$, Arbeitsmedizin ${ }^{34}$ und ein fachdisziplinenübergreifender Katalog der Chirurgie ${ }^{35}$ entstanden. Diesen Lernzielkatalogen ist gemein, dass sie im Gegensatz zum Gegenstandskatalog des IMPP kompetenzorientiert formuliert sind.

\subsection{Kompetenzbasiertes Lernen}

Ärztliche Kompetenz lässt sich definieren als "habitual and judicious use of communication, knowledge, technical skills, clinical reasoning, emotions, values and reflections in daily practice for the benefit of the individual and community being 
served“. ${ }^{36}$ Für den deutschsprachigen Raum definiert Weinert 2001 Kompetenz als „die bei Individuen verfügbaren oder durch sie erlernbaren kognitiven Fähigkeiten und Fertigkeiten, um bestimmte Probleme zu lösen, sowie die damit verbundenen motivationalen, volitionalen (d. h. absichts- und willensbezogenen, E. K.) und sozialen Bereitschaften und Fähigkeiten, um die Problemlösungen in variablen Situationen erfolgreich und verantwortungsvoll nutzen zu können. “"

Der Erwerb dieser Kompetenzen ist hierbei ein Stufenprozess. Wesentliche Voraussetzung für kompetentes Handeln ist Wissen. ${ }^{38,39}$ Es soll gemeinsam mit anwendungsorientiertem Können, überfachlichen Schlüsselkompetenzen sowie persönlicher Motivation und Verantwortung die Basis bilden, um bestimmte ärztliche Tätigkeiten beobachtbar und überprüfbar durchführen zu können. ${ }^{40}$

Dieser Stufenprozess wurde von Miller anschaulich als Pyramide (Abb. 1) beschrieben, diese Ausführungen gelten hier als Standardtaxonomie. ${ }^{39}$

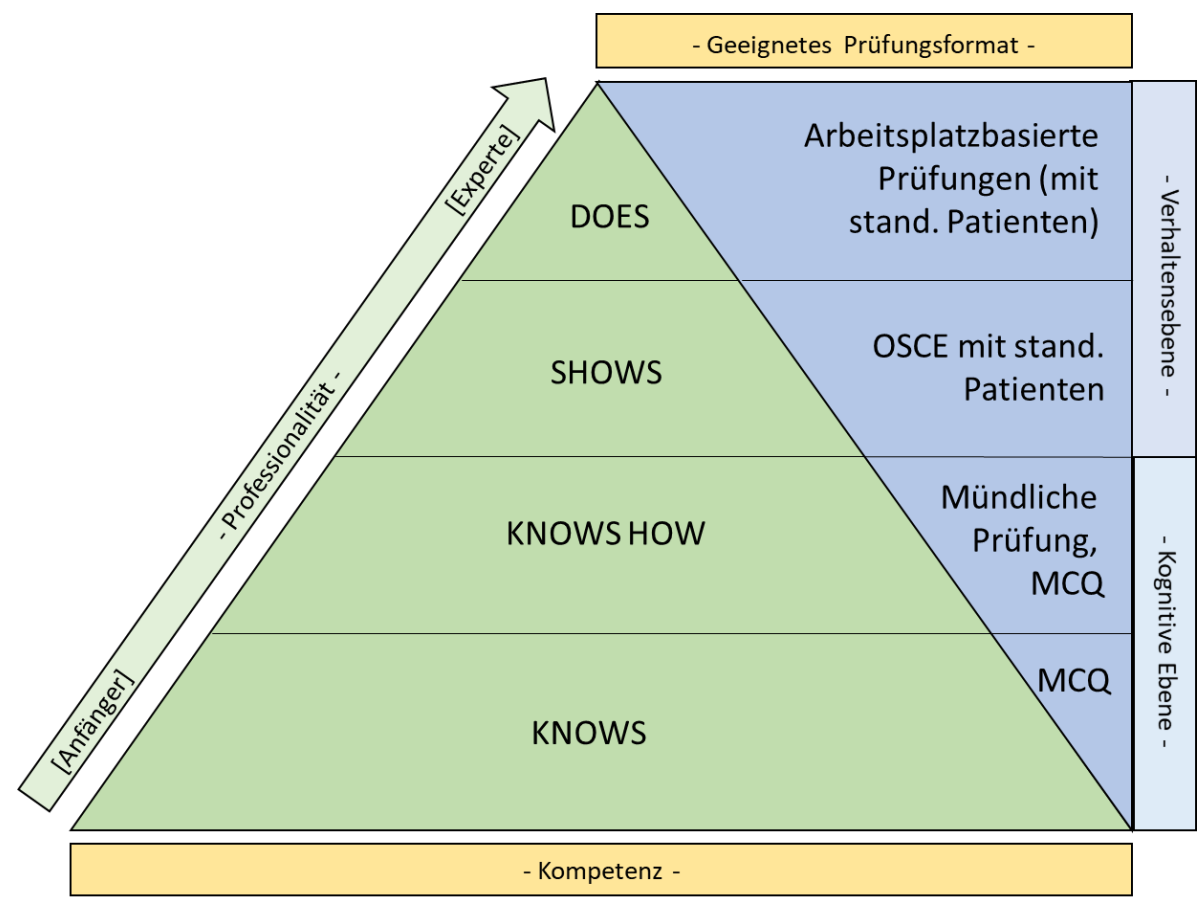

Abb. 1 Modifizierte Pyramide nach Miller ${ }^{39}$ mit geeigneten Prüfungsformaten ${ }^{41,42}$ MCQ = Multiple Choice Questions, OSCE = Objective structured clinical examination

Basierend auf der Millerpyramide wird die "Lerntiefe" durch die Kompetenzebenen gekennzeichnet ${ }^{43}$ Es werden drei Ebenen unterschieden, die aufeinander aufbauen: 
1. Faktenwissen: Deskriptives Wissen (Fakten, Tatsachen) nennen und beschreiben.

2. Handlungs- und Begründungswissen: Sachverhalte und Zusammenhänge erklären, in den klinisch-wissenschaftlichen Kontext einordnen und datenbasiert bewerten.

3. Handlungskompetenz:

3a. Unter Anleitung selbst durchführen und demonstrieren.

3b. Selbstständig und situationsadäquat in Kenntnis der Konsequenzen durchführen. ${ }^{43,12}$

Die Kompetenzebenen des NKLM sind eng an die Miller-Pyramide und die im Swiss Catalogue of Learning Objectives for Undergraduate Medical Training (SCLO) beschriebenen Ebenen angelehnt. Die folgende Abbildung (Abb. 2) zeigt einen Vergleich.

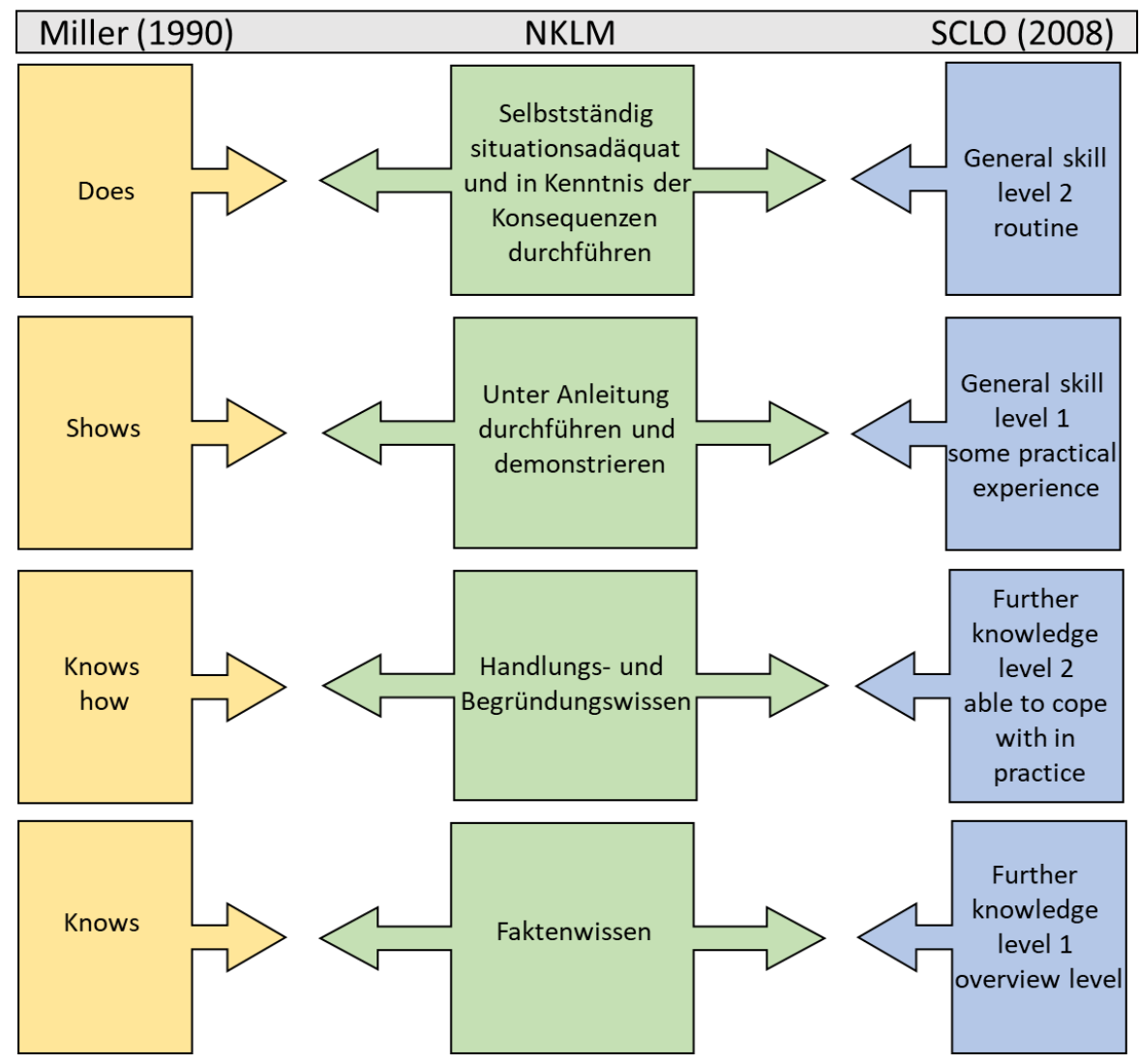

Abb. 2 Vergleich der angewendeten Kompetenzebenen bei Miller, im NKLM und im SCLO. 
Diese und ähnliche Überlegungen sind die Grundlage des bereits seit den 1970er Jahren auf internationaler Ebene diskutierten kompetenzorientierten Ausbildungskonzept. ${ }^{44}$ Die sogenannte Competency-based medical education (CBME) ist auch gegenwärtig Bestandteil umfangreicher Diskussionen. ${ }^{10,45}$ Die in der Literatur beschriebenen Definitionen der CBME weisen eine enorme Heterogenität auf. In ihrer Übersichtsarbeit von 2010 beschreiben Frank et al. eine zusammengefasste, aktuelle Definition als "Competency-based education (CBE) is an approach to preparing physicians for practice that is fundamentally oriented to graduate outcome abilities and organized around competencies derived from an analysis of societal and patient needs. It deemphasizes time-based training and promises greater accountability, flexibility, and learnercentredness". ${ }^{46}$

Eine kompetenzorientierte Ausgestaltung des Medizinstudiums ist mit didaktischen Vorteilen verbunden. Sie erlaubt eine effektivere Verknüpfung von Wissen, praktischen Fertigkeiten und professionellen Haltungen, eine stärkere Konzentration des Lernprozesses auf das übergeordnete Ausbildungsziel und eine höhere Nachhaltigkeit der vermittelten Lerninhalte. ${ }^{47}$ Mit kompetenzorientierten Ausbildungskonzepten sollen Studierende so auf ein Arbeiten in einem immer komplexer werdenden Gesundheitssystem mit sich ändernden Erwartungen von Patienten und der Gesellschaft vorbereitet werden. ${ }^{12,48}$ Kompetenzbasiertes Lernen kann hierbei eine hohe Akzeptanz unter Studierenden und Lehrenden erfahren ${ }^{49}$ und das Interesse am jeweiligen Fach sowie das Verständnis für die Zusammenhänge fördern.

Auch der Masterplan 2020 greift die Notwendigkeit einer Kompetenzorientierung des Medizinstudiums auf und hält fest, dass die Inhalte des Studiums an die in der ÄAppO formulierten Ziele der Ausbildung angepasst werden sollen. So soll die Kompetenzorientierung weiter Einzug in die medizinische Ausbildung erhalten, der Masterplan 2020 bildet somit ein sachgerechtes, zeitgemäßes Fortschreiben der begonnenen Reformprozesse. ${ }^{9,17}$ Zudem schafft die Orientierung an übergeordneten Kompetenzen eine verbindende und einheitliche Grundlage für das Medizinstudium. ${ }^{47}$ 
Um diese Ziele auf nationaler Ebene umzusetzen, bedarf es einiger Voraussetzungen, denn kompetenzbasiertes Lehren und Lernen kann nur mit entsprechenden Entwicklungen in den Fakultäten selbst gelingen. Bestehende Curricula müssen verändert und angepasst werden. Lehrende müssen in der entsprechenden Umsetzung geschult sein, denn kompetenzorientierte Ausbildungsstrategien erfordern auch spezielle didaktische Methoden, nicht nur in Vorlesungen und Seminaren, sondern auch und besonders in der klinischen Lehr-Lern-Umgebung. ${ }^{40}$ Die Ausrichtung des Medizinstudiums auf ein kompetenzorientiertes Ausbildungskonzept ist ein langer und komplexer Prozess. ${ }^{50,51}$

\subsection{NKLM und CanMEDs Rollen}

Im Zuge der Reformen zur kompetenzorientierten Ausgestaltung des Medizinstudiums regte 2009 der Hochschulausschuss der Kultusministerkonferenz der Länder an, einen Fachqualifikationsrahmen für das Medizinstudium zu erstellen. Die Gesellschaft für Medizinische Ausbildung (GMA) und der Medizinische Fakultätentag (MFT) entschieden daraufhin einen kompetenzbasierten Lernzielkatalog für das Studium der Medizin und Zahnmedizin zu erstellen, um den Fakultäten die Weiterentwicklung und Modernisierung ihrer Curricula zu erleichtern. ${ }^{52}$ In einer eigens gebildeten Lenkungsgruppe aus MFT, GMA, der Arbeitsgemeinschaft der Wissenschaftlichen Medizinischen Fachgesellschaften (AWMF), Bundesärztekammer (BÄK), Bundesministerium für Bildung und Forschung (BMBF), Bundesministerium für Gesundheit (BMG), Bundesvertretung der Medizinstudierenden in Deutschland (bvmd), Hochschulrektorenkonferenz (HRK), Kultusministerkonferenz (KMK), Gesundheitsministerkonferenz (GMK) und dem Verband der Universitätsklinika Deutschlands (VUD) wurde dieser Lernzielkatalog ausgearbeitet. In einem Delphi-Prozess konnten die einzelnen Arbeitsgemeinschaften bis 2015 den Nationalen Kompetenzbasierten Lernzielkatalog Medizin (NKLM), auch in Anlehnung an andere internationale Rahmenwerke, ${ }^{10,12,14,53}$ erstellen und veröffentlichen. 
Eine wichtige Grundlage des Prozesses bildeten hierbei die CanMEDS-Rollen. Diese sind in den 1990er Jahren in Kanada aus dem „Educating Future Physicians for Ontario (EFPO) Project" entwickelt worden, ${ }^{54}$ um eine Reformierung der medizinischen Ausbildung für mehr Realitätsnähe zu etablieren. Ursprünglich waren sie für die Weiterbildung auf nationaler kanadischer Ebene bestimmt und fanden international große Resonanz. ${ }^{10,54,55}$

Im weiteren Verlauf fand eine Übertragung auf die Kanadische Weiterbildung und Verankerung im gesamten Gesundheitssystem statt. ${ }^{10}$ Nach weiterer Entwicklung beschreibt das CanMEDS Rahmenkonzept nun für die postgraduale Weiterbildung in Kanada Handlungskompetenzen, welche die Absolventen des Humanmedizinstudiums erworben haben müssen, um den Rollen eines Arztes der heutigen Zeit gerecht werden zu können. Die Einarbeitung dieses Grundkonzepts der ärztlichen Rollen ist einer der zentralen Bestandteile in dem Erstellungsprozess des NKLM. ${ }^{3}$

Mit dem NKLM entstand erstmals in Deutschland ein zwischen den Fachgesellschaften, den Vertretern der Fakultäten, der Studierendenschaft und den politischen Akteuren konsentierter Lernzielkatalog mit Empfehlungscharakter. Der NKLM stellt ein Kerncurriculum dar, das Inhalte des deutschen Medizinstudiums bildet. Als Rahmenwerk soll er erlernbare Kompetenzen und Ausbildungsziele für Lehrende und Lernende transparent abbilden und so die Vorgaben der Approbationsordnung konkretisieren und bündeln. $\mathrm{Er}$ beschreibt mit seinen insgesamt 1956 Lernzielen, 234 Kompetenzen und 281 Teilkompetenzen grundlegende Kenntnisse, Fähigkeiten und Fertigkeiten sowie professionelle Haltungen, über die die Absolventinnen und Absolventen am Ende des Medizinstudiums verfügen sollen. ${ }^{32}$ Er fungiert ergänzend zum Gegenstandskatalog des IMPP, der das unentbehrliche medizinische Fachwissen und damit das Prüfungswissen über systematische Themen-/Begriffslisten definiert. Es besteht eine sehr hohe inhaltliche Übereinstimmung zwischen den beiden Katalogen. Somit kann der NKLM auch Sicherheit bezüglich der vorhandenen Examensrelevanz vermitteln. ${ }^{56}$

Der NKLM ist nach ärztlichen Rollen, Kapiteln und Domänen gegliedert. Er ist in drei 
Abschnitte eingeteilt und umfasst insgesamt 21 Kapitel (Abb. 3). Die ersten 4 Kapitel stellen den Referenzrahmen dar. Der erste Abschnitt (Kapitel 5 bis 11) wird von den übergeordneten Kompetenzrollen des Arztes/der Ärztin in Anlehnung an die CanMEDs Rollen gebildet. ${ }^{10}$ Der zweite Abschnitt (Kapitel 12 bis 19) behandelt das medizinische Wissen, die klinischen Fähigkeiten und professionelle Haltungen. Der dritte Abschnitt (Kapitel 20 und 21) beinhaltet die patientenzentrierte Gesundheitsversorgung durch eine Zusammenstellung von Krankheitsbildern und Konsultationsanlässen. Für deren Management und Behandlung sind die konkreten Handlungskompetenzen der vorherigen Kapitel erforderlich. Der NKLM ist explizit als übergeordneter, weder vorklinischer oder klinischer noch einzelfachbezogener Lernzielkatalog zu verstehen. ${ }^{57}$

\begin{tabular}{|c|c|c|}
\hline Abschnitt I & Abschnitt II & Abschnitt III \\
\hline $\begin{array}{l}\text { Medizinischer } \\
\text { Experte }\end{array}$ & $\begin{array}{l}\text { Prinzipien normaler } \\
\text { Struktur und Funktion }\end{array}$ & \multirow{5}{*}{$\begin{array}{l}\text { Anlässe für } \\
\text { ärztliche } \\
\text { Konsultation }\end{array}$} \\
\hline \multirow{2}{*}{ Gelehrter } & $\begin{array}{l}\text { Pathogenese und } \\
\text { Pathomechanismen }\end{array}$ & \\
\hline & $\begin{array}{c}\text { Medizinisch-wissen- } \\
\text { schaftliche Fertigkeiten }\end{array}$ & \\
\hline Kommunikator & $\begin{array}{l}\text { Klinisch praktische } \\
\text { Fertigkeiten }\end{array}$ & \\
\hline \multirow{2}{*}{ Mitglied eines Teams } & $\begin{array}{c}\text { Ärztliche } \\
\text { Gesprächsführung }\end{array}$ & \\
\hline & $\begin{array}{l}\text { Diagnostische } \\
\text { Verfahren }\end{array}$ & \multirow{6}{*}{$\begin{array}{c}\text { Erkrankungsbezogene } \\
\text { Prävention, } \\
\text { Diagnostik, } \\
\text { Therapie, } \\
\text { Versorgungs- } \\
\text { und } \\
\text { Notfallmaßnahmen }\end{array}$} \\
\hline $\begin{array}{l}\text { Gesundheitsberater/ } \\
\text {-fürsprecher }\end{array}$ & $\begin{array}{l}\text { Therapeutische } \\
\text { Prinzipien }\end{array}$ & \\
\hline \multirow{2}{*}{$\begin{array}{c}\text { Verantwortungsträger } \\
\text { und Manager }\end{array}$} & Notfallmaßnahmen & \\
\hline & Ethik, Geschichte & \\
\hline \multirow[b]{2}{*}{$\begin{array}{l}\text { Professionell } \\
\text { Handelnder }\end{array}$} & und Recht der Medizin & \\
\hline & $\begin{array}{c}\text { Gesundheitsförderung } \\
\text { und Prävention }\end{array}$ & \\
\hline
\end{tabular}

Abb. 3 Aufbau der Abschnitte 1 - 3 des NKLM

Innerhalb der Kapitel und Unterkapitel wurde eine dreistufige, hierarchische Gliederung eingesetzt: Ebene 1 bildet Kompetenzen ab, auf Ebene 2 werden Teilkompetenzen formuliert und auf Ebene 3 sind Lernziele mit Angabe der Kompetenzebenen festgehalten. Die Ebenen 1 und 2 haben Empfehlungscharakter. 
Die Ebene 3 soll von den medizinischen Fakultäten erprobt und kritisch evaluiert werden. ${ }^{32}$

\begin{tabular}{|c|c|c|}
\hline $\boldsymbol{\Delta}$ & & GK BK PJK WK WissK \\
\hline 12.1 & $\begin{array}{l}\text { Die Absolventin und der Absolvent erklären, erläutern und schlussfolgern auf wissenschaftlicher Grundlage unter Verwendung } \\
\text { medizinischer Terminologie. Sie können ... } \bullet \text {. }\end{array}$ & \\
\hline 12.1.1.1 & $\begin{array}{l}\text { den Aufbau des Körpers und die wesentlichen Vorgänge im Körper, im Erleben und Verhalten erklären und dabei } \\
\text { geschlechtsspezifische, altersspezifische und kulturelle Aspekte berücksichtigen. } \odot \text {. }\end{array}$ & 2 \\
\hline 12.1.1.2 & die Funktionen aus den zu Grunde liegenden Strukturen ableiten. $\odot$ & 2 \\
\hline 12.1.1.3 & die Bedeutung von individueller Variabilität und Kriterien für Normalität erläutern. $\odot$ & 2 \\
\hline 12.1.1.4 & differentielle Einflüsse auf Strukturen, Funktionen, Erleben und Verhalten erklären. $\odot$ & 2 \\
\hline 12.1.1.5 & Prinzipien aus den zu Grunde liegenden Strukturen und Vorgängen ableiten. $\odot$. & 2 \\
\hline 12.1.1.6 & Prinzipien verwenden, um Funktionsstörungen zu erklären und Symptome sowie Therapiemöglichkeiten abzuleiten. $\odot$. & 2 \\
\hline 12.2 & $\begin{array}{l}\text { Die Absolventin und der Absolvent erklären, auf welchen Grundlagen Vorgänge im menschlichen Körper und in sozialen } \\
\text { Gruppen beruhen. } \bullet \text {. }\end{array}$ & \\
\hline 12.2 .1 & Sie erklären, auf welchen physikalischen Grundlagen Vorgänge im menschlichen Körper beruhen. Sie können ... $९$. & \\
\hline 12.2 .1 .1 & Regelprozesse erklären und Regelprinzipien anwenden. $९$. & 2 \\
\hline 12.2.1.2 & die Mechanik starrer und deformierbarer Körper erklären. $\odot$ & 2 \\
\hline 12.2.1.3 & die Grundlagen der Wärmelehre erklären. $\odot$. & Ebene 3 \\
\hline 12.2.1.4 & die Bedeutung von Schwingungen und Wellen in der Medizin erklären. $९$ & 2 \\
\hline
\end{tabular}

Abb. 4 Auszug aus Kapitel 12 des NKLM. Darstellung der hierarchischen Gliederungsebenen $1-3$.

Auf Ebene 3 werden zu den Lernzielen, die auf einander aufbauenden Kompetenzebenen 1, 2, 3a und 3b angegeben, die die Absolventen erreichen sollen. Auf dieser Ebene wird auch konkretisiert, zu welchem zeitlichen Abschnitt der Absolvent (mit einem gewissen Gestaltungsspielraum) das jeweilige Lernziel mit der entsprechenden Kompetenzebene erreicht haben soll. Dazu werden im NKLM 5 Meilensteine formuliert: Grundlagenkompetenzen GK (in Regelstudiengängen mit Abschluss M1 erreicht, in Modellstudiengängen spätestens vor Eintritt in das Praktische Jahr (PJ)), Ärztliche Basiskompetenzen BK für die Ausbildung mit unmittelbarem Patientenbezug (in der Regel zwischen M1 und M2 erreicht), PJKompetenz PJ (erreicht mit M2 als PJ-Kompetenzprüfung und Zulassung zum PJ), Ärztliche Approbation und Weiterbildungskompetenz WK (mit M3 erreicht) und Wissenschaftskompetenz WissK (implizit erreicht mit Abschluss des Universitätsstudiums).

Die in Abschnitt 1 des NKLM dargestellten übergeordneten ärztlichen Kompetenzrollen orientieren sich an den kanadischen CanMEDS Rollen. Der NKLM 
formuliert folgende sieben ärztliche Rollen, diese bilden gleichzeitig die Kapitel 5 11:

\begin{tabular}{|ll|}
\hline Kapitel & \\
\hline $\mathbf{5}$ & Die Ärztin / der Arzt als Medizinische Experteninnen / Medizinische Experten \\
$\mathbf{6}$ & Die Ärztin / der Arzt als Gelehrte / Gelehrter \\
$\mathbf{7}$ & Die Ärztin / der Arzt als Kommunikatorinnen / Kommunikatoren \\
$\mathbf{8}$ & Die Ärztin / der Arzt als Mitglieder eines Teams \\
& Die Ärztin / der Arzt als Gesundheitsberaterin und -fürsprecherin / \\
$\mathbf{9}$ & Gesundheitsberater und -fürsprecher \\
& Die Ärztin / der Arzt als Verantwortungsträgerin und Managerin / \\
$\mathbf{1 0}$ & Verantwortungsträger und Manager \\
$\mathbf{1 1}$ & Die Ärztin / der Arzt als Professionell Handelnde / Professionell Handelnder \\
\hline
\end{tabular}

Tab. 1 Auflistung der Kapitel des Abschnitts 1.

Diese ärztlichen Kompetenzrollen sind in ihrer Darstellung für die berufliche Praxis 58-59 und für die vom Wissenschaftsrat geforderten „sozialen, kommunikativen, klinisch-praktischen und wissenschaftlichen Kompetenzen" hochrelevant. ${ }^{47}$ Sie sind bewusst schon während des Medizinstudiums und während des PJ und nicht erst in der Weiterbildung relevant und können dort in den Unterricht konkret eingebaut werden. ${ }^{60}$ Die ärztlichen Kompetenzen sollen nicht isoliert als „Add on“, sondern in praktischen Kontexten, verwoben mit Leitsymptomen und Krankheitsbildern oder auch in Verbindung mit theoretischen Konzepten und Prinzipien, vermittelt werden. ${ }^{61}$ Von Lehrenden werden die Arztrollen als absolut praxis- und alltagsrelevant eingestuft. ${ }^{60,62}$ Die exakte Rollenbeschreibung lässt sich jedoch nicht immer intuitiv erfassen und bedarf zum vollen Verständnis und bei Missverständnissen professioneller Erklärung. ${ }^{62,63}$

In Abschnitt 2 des NKLM wird der Umfang der medizinischen Expertise weiter konkretisiert. Dabei werden relevantes Wissen und wissenschaftliche Grundlagen zum Erkenntnisgewinn, klinische Fähigkeiten und Fertigkeiten in Prävention, Diagnose und Therapie sowie ärztliche Grundhaltungen ausformuliert. Auch hier werden Anwendungsbezüge zu Abschnitt 3 mit Anlässen für ärztliche Konsultationen und zu Erkrankungen hergestellt. 
Die Überschriften der Kapitel 12 - 19 in Abschnitt 2 lauten:

\begin{tabular}{|rl|}
\hline Kapitel & \\
\hline 12 & Prinzipien normaler Struktur und Funktion \\
13 & Prinzipien der Pathogenese und Pathomechanismen \\
$14 a$ & Medizinisch-wissenschaftliche Fertigkeiten \\
\hline $14 b$ & Klinisch-praktische Fertigkeiten \\
14 c & Ärtliche Gesprächsführung \\
15 & Diagnostische Verfahren \\
16 & Therapeutische Prinzipien \\
17 & Notfallmaßnahmen \\
18 & Ethik, Geschichte und Recht \\
19 & Gesundheitsförderung und Prävention \\
\hline
\end{tabular}

Tab. 2 Auflistung der Kapitel des Abschnitt 2

Abschnitt 3 des NKLM beschreibt wichtige Anlässe für ärztliche Konsultationen sowie relevante exemplarische Krankheitsbilder. Auswahlkriterien zur Aufnahme in den Katalog sind insbesondere die Häufigkeit des Auftretens, eine hohe akute Letalität, die Abwendbarkeit von Tod und bleibender Gesundheitsschädigung durch Intervention, eine langfristige Einschränkung der Lebensqualität und die Transferierbarkeit von Prinzipien. ${ }^{3}$ Auch seltene Erkrankungen finden exemplarisch Erwähnung und sollen die notwendigen Kompetenzen im Umgang mit seltenen Erkrankungen und der methodische Zugang zu spezifischen Informationsquellen und -techniken fördern. So beinhaltet der dritte Abschnitt die Kapitel 20 und 21 mit den folgenden Überschriften:

\begin{tabular}{|ll|}
\hline \multicolumn{1}{|l}{ Kapitel } & \\
\hline $\mathbf{2 0}$ & Anlässe für ärztliche Konsultation \\
& Erkrankungsbezogene Prävention, Diagnostik, Therapie, Versorgungs- und \\
$\mathbf{2 1}$ & Notfallmanagement. \\
\hline
\end{tabular}

Tab. 3 Auflistung der Kapitel des Abschnitt 3

Der NKLM formuliert bewusst keine fächer- und organsystemspezifische Zuordnung oder Zuordnungsempfehlungen. Damit stellt der NKLM die Universitäten vor didaktische Herausforderungen. Die Erstellung von fachspezifischen Lernzielkatalogen obliegt zudem den jeweiligen Fachgesellschaften. ${ }^{33}$ Es existieren bereits fächerspezifische Lernzielkataloge, die von Vertretern der einzelnen Fachgesellschaften entwickelt und an den NKLM angelehnt sind, wie zum Beispiel 
der Nationalen Kompetenzbasierten Lernzielkatalog der Chirurgie (NKLC) ${ }^{64}$, der Lernzielkatalog Arbeitsmedizin ${ }^{34}$ oder der der Psychosomatik ${ }^{33}$. Diese fächerspezifischen Lernzielkataloge sollen als Bindeglied zwischen den Curricula der einzelnen Fakultäten und dem NKLM fungieren. So soll beispielsweise der NKLC die Umsetzung eines chirurgischen Kernkurrikulums für die Studierenden unterstützen. Dem Fach soll somit eine besondere Sichtbarkeit in der studentischen Ausbildung verliehen und gleichzeitig Überfrachtung vermieden werden. Der NKLC beschreibt daher ausdrücklich das minimale Kompetenzprofil von Ärztinnen und Ärzten in den chirurgischen Fachdisziplinen zum Zeitpunkt der Approbation. ${ }^{57}$ Auch Fakultätsinterne Curricula sind zur Umsetzung des NKLM erforderlich. Sie sollen anhand des NKLM und daran angelehnter fachspezifischer Curricula adaptiert und ausgebaut werden. ${ }^{64}$ Studien- und Prüfungsordnungen der Fakultäten sollen wie bisher weiterhin verbindlich bleiben. ${ }^{3}$ Die Formulierung der (Teil-) Kompetenzen und Lernziele, im Gegensatz zur Auflistung von Fachwissen in systematischen Begriffslisten (wie im GK), erleichtert den Fakultäten die Gestaltung eigener Lernzielkataloge und sichert vor allem die Äquivalenz der Ausbildung an verschiedenen Standorten. ${ }^{56}$

Die Herausforderungen in der Umsetzung des NKLM sind vor allem darin zu sehen, dass die kontinuierliche Anpassung der Lernziele unter wissenschaftlichen Gesichtspunkten unter Federführung der Fakultäten erfolgen muss. ${ }^{65}$ Nur so ist eine breite Akzeptanz gewährleistet und die Profil- und Schwerpunktbildung der einzelnen Fakultäten kann weiterhin ermöglicht werden. Eine Verankerung der Kompetenzorientierung erfordert gegebenenfalls neue Lehr- und Prüfungsformate. Auch deren Etablierung muss wissenschaftlich begleitet, sorgfältig evaluiert und auf Umsetzbarkeit überprüft werden. ${ }^{66}$

Mit der Erstellung des NKLM ist ein Rahmen geschaffen worden, der die Anforderungen eines Medizinstudiums in Deutschland so definiert, dass ein europarechtlichen Vorgaben genügender und qualitätsgesicherter Zugang zum Beruf der Ärztin / des Arztes gewährleistet ist. ${ }^{3}$ Er soll helfen, Überfrachtung in der medizinischen Ausbildung zu vermeiden und Zeiträume für die praktische, patientenorientierte Ausbildung im klinischen Umfeld zu schaffen. Er soll es 
ermöglichen, Ärztinnen und Ärzte der Zukunft bestmöglich auszubilden, sodass sie den Herausforderungen im Gesundheitssystem zum Wohle der Patientinnen und Patienten in all ihren verschiedenen Rollen gerecht werden können und soll den Übergang zur klinischen Weiterbildung erleichtern. ${ }^{32}$

Bis 2020 findet eine Erprobung und Review der Inhalte des NKLM an den klinischen Fakultäten statt. ${ }^{32}$ Der NKLM ist weiter als „work in progress“ zu verstehen, der wie jedes Curriculum einer ständigen Verbesserung und Aktualisierung bedarf. ${ }^{33}$ Die Weiterentwicklung des NKLM zum NKLM „2.0“ erfolgt aktuell weiterhin auf Basis der im Masterplan 2020 festgehaltenen Reformziele. ${ }^{17,67}$ Es soll dabei der Fokus vor allem auf die Verknüpfung von grundlagenwissenschaftlichen und klinischen Inhalten, sowie klinisch-praktischen Fertigkeiten über alle Studienphasen hinweg, gelegt werden. Ein weiterer wichtiger Bestandteil ist die Abstimmung der Ausgestaltung mit dem Gegenstandskatalog (GK) des IMPP, um eine Übereinstimmung von Studieninhalten und Prüfungsinhalten zu gewährleisten. ${ }^{7}$

Damit der curriculare Wandel zur Kompetenzorientierung mithilfe der Vorgaben des NKLM als Orientierungsrahmen stattfinden kann, ist eine Begleitung des Prozesses durch ein effektives Changemanagement ist zwingend notwendig. Die Durchführung eines Curriculum Mapping hat sich an dieser Stelle als sinnvoll erwiesen. ${ }^{65}$

\subsection{Mapping}

Unter dem Begriff Curriculum Mapping versteht man die Katalogisierung von gelehrten Inhalten einer bestimmten universitären Lehrveranstaltung (Vorlesung, Seminare, Praktika, etc.). ${ }^{61}$ Das sogenannte Mapping ist ein etabliertes Verfahren, findet sowohl deutschlandweit als auch international an medizinischen Fakultäten Anwendung und ist ein wichtiger Bestandteil der Lehreforschung. Im nationalen Rahmen dient es im weitesten Sinne der Erhebung der im Medizinstudium gelehrten Inhalte auf Grundlage des NKLM.

Im Rahmen des Mappings entsteht eine curriculare Kartierung, die abbildet was, wann, wie und von wem gelehrt und gelernt wird. Die wichtigste Funktion des Mappings ist es, gelehrte Inhalte bzw. das Curriculum transparent zu machen und so ein aufeinander abgestimmtes Ausbildungsprogramm zu schaffen. ${ }^{68-69}$ 
Hierbei unterscheiden sich folgende Arten des Curriculums, die sich aus verschiedenen Sichtweisen auf das Curriculum ergeben:

-Declared curriculum: Es umfasst die in schriftlichen Unterlagen wie Vorlesungsverzeichnissen, Handbüchern, Modulleitfäden und Lernzielkatalogen festgehaltenen Inhalte und ist explizit darstellt.

-Taught curriculum: Beschreibt die aus Sicht der Lehrenden vermittelten Inhalte und kann auch implizit formuliert sein.

-Learned Curriculum: Beinhaltet aus Sicht der Studierenden, was gelernt wurde und sich oft in Prüfungen wiederspiegelt. ${ }^{61}$

Die Inhalte sind sehr oft nicht deckungsgleich, was sich aufgrund eines Mangels an Abstimmung oder eines Mangels an Einsicht über die Inhalte von Prüfungen ergibt. Eine möglichst große Schnittmenge sollte jedoch immer angestrebt werden. ${ }^{61,70}$

Eine Durchführung des Curriculum Mappings soll dabei helfen Lücken und Redundanzen im Curriculum aufzudecken. ${ }^{71}$ Ebenso lassen sich damit Querverbindungen zwischen Fachwissen und longitudinalem Kompetenzerwerb visualisieren und Inkonsistenzen in Inhalt, Lerngelegenheiten oder Prüfungen können aufgedeckt werden. ${ }^{72,73}$

Eine Statuserhebung im Rahmen des Curriculum Mapping kann und soll in einem Prozess mit einer Curriculumsentwicklung und -planung verknüpft werden. ${ }^{74,71}$ Eine weitere wichtige Voraussetzung für den Wandel hin zur kompetenzorientierten Ausbildung ist es, eine angemessene curriculare Transparenz für Lehrende, Studierende und Administration zu schaffen. Ohne diese ist ein systematischer Aufbau eines komplexen Curriculums kaum möglich. ${ }^{61,71}$ Ein Mapping anhand des NKLM kann für die nötige Transparenz sorgen und zeigen, wo curriculare Optimierungen möglich und nötig sind. ${ }^{65}$

Eine bundesweite Statuserhebung mittels des Curriculum Mappings erscheint sinnvoll, da an den einzelnen Fakultäten oft komplexe, dezentral gewachsene Curricula existieren, ${ }^{74}$ die nur gering in Lehrinhalten und Fächern abgestimmt sind. Eine nationale Vergleichbarkeit kann so erzielt werden. ${ }^{72}$ Die Umsetzung der „Praxisnähe“ im Studium und Reformen müssen wissenschaftlich begleitet werden. ${ }^{75}$ 
Um ein so umfangreiches Projekt wie das NKLM Curriculum Mapping möglichst flächendeckend erfolgreich durchzuführen, erfordert es geschultes Personal zur Koordination, tragfähige Kommunikationsstrukturen, sowie eine bedarfsorientierte Mapping-Datenbank. ${ }^{65}$ Dazu erhielten die Fakultäten Freiburg, Heidelberg, Mannheim, Tübingen und Ulm 2012 eine fünfjährige Förderung, um auf Basis des NKLM Maßnahmen zur kompetenzorientierten Umgestaltung des Medizinstudiums entwickeln zu können. Es entstand im Rahmen des BMBF-Verbundprojekt Medical Education Research - Lehreforschung im Netz BW (MERLIN) ${ }^{76,77}$ unter anderem eine web-basierten Datenbank zur Durchführung eines Curriculum Mappings anhand des NKLM.

Mit den im Verbundprojekt MERLIN erhobenen Daten kann die longitudinale Entwicklung der NKLM-Rollen und Teilkompetenzen visualisiert werden, wobei Umfang, Niveau und Prüfungsformat dargestellt werden können. Es kann gezeigt werden, welche Fächer mit welchen Lehrveranstaltungen an der Vermittlung definierter Rollen implizit und explizit beteiligt sind. ${ }^{78}$ Das Curriculum kann so transparenter gemachen werden und einzelne Fächer in das Gesamtfeld eingeordnet werden. So wird eine Anregung geschaffen fächerübergreifend zu kommunizieren und Lehrinhalte abzugleichen. ${ }^{63}$ Somit ist ein Medium geschaffen worden, das das Mapping praktikabel und produktiv macht und zudem Daten liefert, die zielgerichtet, belastbar und schnell erfassbar sind. Dies schafft eine Grundlage eine allgemeine Bereitschaft und Akzeptanz bei allen an der Lehre der Fakultät beteiligten, um ein Curriculum Mapping durchzuführen. ${ }^{78,79}$ So erhält man eine notwendige Datenbasis, um ein nachhaltiges curriculares Gesamtkonzept zu entwickeln. ${ }^{65}$ Die Erfahrungen und Empfehlungen aus dem Tübinger Konzept bezüglich des Mappings konnten an anderen bundesweiten Standorten und Fakultäten implementiert werden und wurden von Lammerding-Koeppel et al. als „10 Empfehlungen für den Mapping-Koordinator" formuliert und festhalten. ${ }^{65}$ Curriculum Mapping stellt an den einzelnen Fakultäten und bundesweit das zentrale Werkzeug dar, bestehende Curricula abzubilden und diese gezielt weiter zu entwickeln oder umzustrukturieren, und so die Kompetenzorientierung 
flächendecken zu verankern. Ein erfolgreicher Change-Prozess bedarf einer strukturierten Führung, eines kooperativen Klimas und der Einbindung von Organisationsmitgliedern, Politik, Personalentwicklung und Evaluation. ${ }^{63,80}$ An der Frankfurter Johann Wolfgang Goethe Universität haben bereits einzelne Projekte zum fakultätsinternen Curriculum Mapping stattgefunden. So konnten fachspezifisch für die Vorlesungen der Chirurgie gezeigt werden, dass ein Mapping Projekt die Anzahl der gelehrten Lernziele im Rahmen des "taught curriculums“ erhöhen kann. ${ }^{81}$ Auch das zahnmedizinische Ausbildung in Frankfurt konnte mittels Mapping detailliert abgebildet und daraus ein fakultätsinternes Curriculum abgeleitet und weiterentwickelt werden. ${ }^{82}$ Die Anwendung des Curriculum-Mapping auf ein Blockpraktikum, wie es in dieser Studie beschrieben wird, ist im Vorfeld noch nicht erfolgt. Es stellt mit einer Ausrichtung an verschiedenen Kliniken und unterschiedlichen chirurgischen Fachrichtungen eine besondere Herausforderung dar.

\subsection{Chirurgisches Curriculum Frankfurt am Main}

Am Fachbereich Medizin an der Goethe Universität beginnt die Ausbildung vom Fach Chirurgie im 1. Semester des klinischen Studienabschnitts. Grundlage sind die Inhalte des fakultätsinternen Lernzielkatalogs. ${ }^{3,30} \mathrm{Er}$ beinhaltet die für die studentische Lehre relevanten Krankheitsbilder und Symptome sowie praktische Fertigkeiten und Fähigkeiten und allgemeine, übergeordnete Lernziele. Jedem Lernziel wird eine Kompetenzebene $1-3 b$ zugewiesen. Diese legt fest, in welche Tiefe ein Lernziel vermittelt und erlernt werden soll. Die Definition der einzelnen Kompetenzebenen ähnelt denen des NKLM und NKLC. Auf Kompetenzebene $2-$ $3 b$ wird eine Bestimmung der Kompetenzbereiche T, D, M, N vorgenommen (Therapie, Diagnostik, Management, Notfall). Zudem folgt eine Zuordnung der

jeweiligen Lernziele zu den lehrenden chirurgischen Fächern und eine Zuordnung zu möglichen Verknüpfungen mir interdisziplinären Fächern.

Im 1. Klinischen Semester findet die Vorlesungsreihe Einführung in die Chirurgie statt. Hier werden die chirurgischen Grundlagen wie beispielsweise die Prinzipien der Wundheilung und des Verhaltens im OP vermittelt. Den Abschluss bildet eine 
aus Multiple-Choice Fragen bestehende Semesterabschlussklausur. Ab dem 2. Klinischen Semester werden die Studierenden in zwei Teilkohorten aufgeteilt, die sich entweder erst Hauptvorlesung der Inneren Medizin oder der Chirurgie widmen. Im zweiten oder dritten Semester (je nach Teilkohorte) findet die Hauptvorlesung Chirurgie statt. Diese besteht aus einer weiteren Reihe von 32 Vorlesungen aller chirurgischen Fachdisziplinen. Zudem müssen die Studierenden das 3-wöchige Blockpraktikum Chirurgie absolvieren. Das Blockpraktikum besteht aus dem einwöchigen TpF-Kurs (Training praktischer Fertigkeiten) und dem zwei-wöchigen Stationspraktikums. Im TpF-Kurs erlernen und üben die Studierenden praktische Fertigkeiten wie beispielsweise die Erhebung einer symptomorientierten Anamnese und das Legen eines Venenzuganges, die sie dann im Stationspraktikum anwenden und vertiefen..$^{83}$ Das Stationspraktikum absolvieren die Studierenden an der Universitätsklinik oder einem der akademischen Lehrkrankenhäuser. Die Einteilung hierfür erfolgt, wenn möglich an den Wünschen der Studierenden orientiert, durch das Dekanat. Den Abschluss des Semesters bildet eine aus MC-Fragen bestehende Abschlussklausur und eine Objective Structured Clinical Examination (OSCE) Prüfung mit 8 Stationen, in denen die Lernziele gemäß des Prinzips des "Constructive alignment“27 überprüft werden.

Nach Abschluss des zweiten beziehungsweise dritten Semesters sieht die Studienordnung die nächste chirurgische Pflichtveranstaltung für Studierende erst wieder als Pflichttertial des Praktischen Jahres vor. Die im Rahmen dessen vorgesehenen Seminare obliegen der Durchführung des jeweiligen Lehrkrankenhauses, in dem die Studierenden ihr PJ ableisten, sind also nicht mehr standardisiert.

Mit Betonung der praxisorientierten und patientennahen Ausbildung der Studierenden in $\S 2$ Absatz 3 der ÄApprO ist den Studierenden ausreichend Gelegenheit zu geben, unter Anleitung, Aufsicht und Verantwortung des ausbildenden Arztes am Patienten tätig zu werden, soweit dies zum Erwerb von Fähigkeiten und Fertigkeiten erforderlich ist. Hier zeigt sich die Bedeutung des Blockpraktikums Chirurgie, um dem Studierenden praktische Fertigkeiten im Klinikumfeld zu vermitteln. Die Chirurgie kann Studierende im Rahmen des 
Studiums und des Pflichttertials im PJ umfassend ausbilden und ist somit prädestiniert eine Vielzahl klinischer Fertigkeiten und Kompetenzen zu vermitteln. ${ }^{84}$ Je nach Lehrkrankenhaus werden die Studierenden in Frankfurt im Blockpraktikum Chirurgie für zwei Wochen einer chirurgischen Fachabteilung zugeordnet oder rotieren durch unterschiedliche Fachabteilungen. Die Vermittlung der Inhalte für die Stationswochen obliegt den jeweiligen Lehrkrankenhäusern. Hierfür speziell existieren keine Vorgaben zu definierten Lernzielen. Als Richtschnur haben die Studierenden jedoch ein Testatheft $z u$ bearbeiten und einzureichen. Dieses Testatheft beinhaltet unter anderem das Erstellen eines Portfolios zu Ambulanzpatienten, beigewohnten Operationen und Verbandswechseln (siehe Anhang). Zwar erfolgt am Ende das Praktikums eine Evaluation durch die Studierenden, diese ermöglicht aber bisher keinen Aufschluss darüber, welche Inhalte die Studierenden im Blockpraktikum erleben. 


\section{Fragestellung}

Ziel der vorliegenden Arbeit ist daher die Durchführung eines Curriculum Mappings des Blockpraktikums Chirurgie mit Fokus auf das Learned Curriculum aus Sicht der Studierenden. Hierbei soll zudem untersucht werden, ob es Unterschiede in Abhängigkeit zu spezifischen Fachabteilungen und Lehrkrankenhäusern gibt und welchen Einfluss das Geschlecht der Studierenden auf die Kompetenzwahrnehmung hat.

Hierbei kann so eine Kartierung des Learned Curriculum erfolgen und mit dem Declared Curriculum abgeglichen werden. 


\section{Material und Methoden}

\subsection{Studiendesign}

Bei der vorliegenden Studie handelt es sich um eine prospektive Lehrforschungsstudie, die mit der Methode des "Curricular Mapping“ die im Blockpraktikum Chirurgie vermittelten Lernziele des NKLM in Abhängigkeit der unterschiedlichen Lehrkrankenhäuser und chirurgischen Fachrichtungen erfasst und analysiert.

Die Durchführung der Studie erfolgte gemäß den Vorgaben der Deklarationen von Helsinki und den Anforderungen des $\$ 15$ der Berufsordnung hessischer Ärzte, jedoch war gemäß der Ethik-Kommission des Fachbereichs Medizin der Goethe Universität Frankfurt am Main kein Ethikvotum erforderlich.

\subsection{Studienteilnehmende}

Studienteilnehmende waren Studierende des 2. klinischen Semesters, die die Hauptgruppe "Chirurgie" mit der Vorlesung Chirurgie II belegen und konsekutiv das Blockpraktikum Chirurgie curricular absolvieren. Das Blockpraktikum Chirurgie ist nach § 27 Absatz 4 Bestandteil zur Zulassung zum zweiten Abschnitt der ärztlichen Prüfung und findet an der Goethe Universität Frankfurt im Anschluss an das 2. bzw. 3. klinische Semester statt. Das Blockpraktikum Chirurgie gilt mit der erfolgreichen Teilnahme an einer im Anschluss stattfindenden OSCE Prüfung gemäß §22 der aktuell gültigen Studienordnung als bestanden. Prüfungsrelevant sind generell die Inhalte des fakultätsinternen Lernzielkatalogs der Chirurgie.

\subsection{Studienprotokoll}

\subsubsection{Studienvorbereitung}

\section{Erstellung eines onlinebasierten Fragebogens}

Zur Erhebung der Daten wurde auf Grundlage des NKLMs eine onlinebasierte Auflistung der im NKLM beschriebenen Lernziele erstellt. Dazu wurde die kostenpflichtige Umfragesoftware SurveyMonkey (Survey Monkey Europe UC, Dublin, Ireland) verwendet. Die Anordnung der Lernziele erfolgte nach den 
Vorgaben der Einteilung des NKLMs in Kapitel und Unterkapitel. So entstand ein Katalog mit 19 Kapiteln (Kapitel 5 bis 21), und insgesamt 2106 einzelnen Lernzielen. Je Lernziel mussten die Studienteilnehmer die folgenden drei Fragen mit jeweils vorgegebenen Antwortmöglichkeiten:

1. Ist das Lernziel erreicht worden?

$\rightarrow \mathrm{JA} / \mathrm{NEIN}$

$\rightarrow$ wenn ja war der Studienteilnehmer angehalten zwei weitere Fragen beantworten.

2. Von wem ist das Lernziel vermittelt worden?

$\rightarrow$ ARZT/KOMILLITONE/PFLEGE

3. Wie erfolgte die Vermittlung?

$\rightarrow$ IMPLIZIT/EXPLIZIT

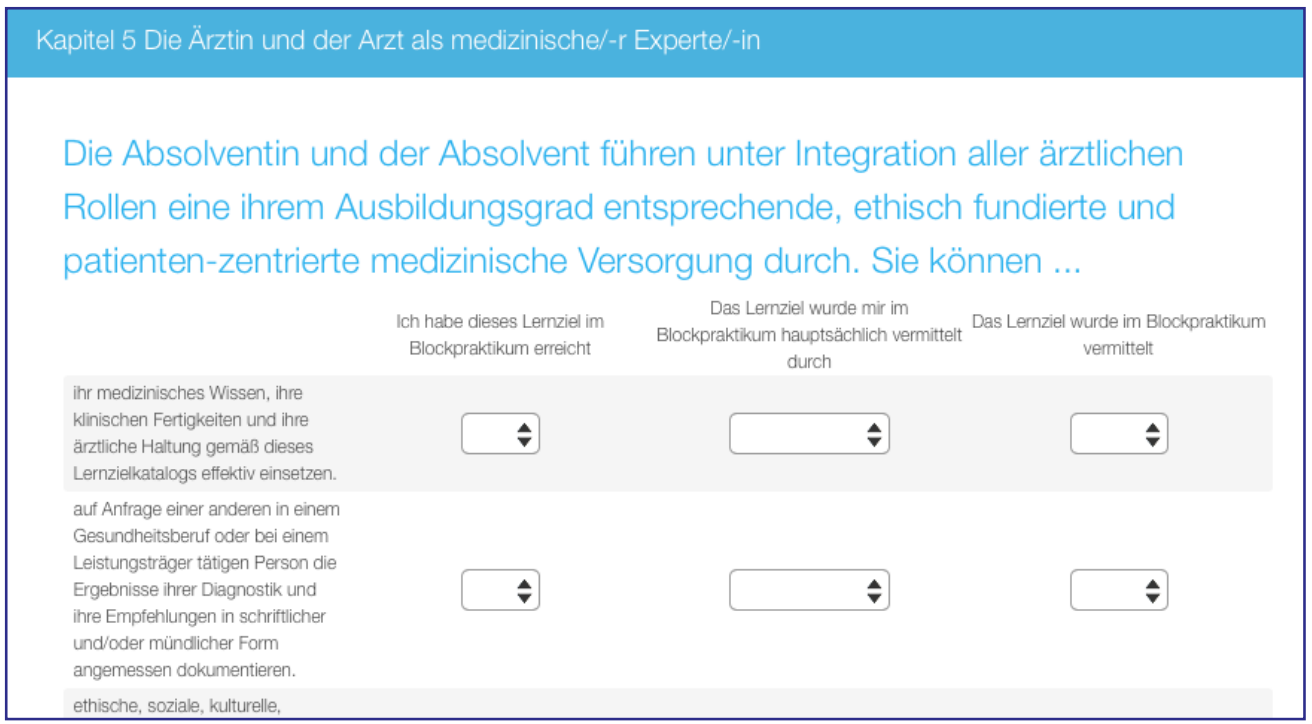

Abb. 5 Layout Onlinebasierter Fragekatalog Survey Monkey

Ergänzt wurde dies durch eine Erhebung der demografischen Daten der Studienteilnehmer. Hierbei wurden Alter, Geschlecht und aktueller Studienstand der Studierenden, Vorkenntnisse zum Beispiel im Rahmen einer medizinischen Ausbildung, sowie die Fachrichtung und das Lehrkrankenhaus, in dem das Blockpraktikum absolviert wurde, erhoben. 
In unserem Projekt zum Kompetenzerwerb im Blockpraktikum Chirurgie findet das Mapping Konzept in leicht modifizierter Form Anwendung: In Bezug auf die hierarchische Gliederung der Unterkapitel des NKLM nach Ebene 1 (Kompetenzen), Ebene 2 (Teilkompetenzen) und Ebene 3 (Lernziele mit Angabe der Kompetenzebenen) formulierten wir die Abfrage in unserem Onlinefragebogen nur auf Ebene 3.Damit konnten wir den Studierenden erleichtern, sich an die im BP vermittelten Lernziele zu erinnern. Eine Erhebung der Kompetenzebenen $1-3 b$ der einzelnen Lernziele fand aus Gründen der Übersicht nicht statt.

\subsubsection{Bearbeitung des Lernzielbogens}

Für die Bearbeitung wurden in einem Zeitraum von maximal 3 Wochen nach Absolvieren des Blockpraktikums Gruppen- und Individualtermine mit den Teilnehmern abgestimmt. Die Studienteilnehmer bearbeiteten die Umfrage individuell unter Aufsicht und ausführlicher Erklärung eines geschulten Studienmitarbeiters in den Räumlichkeiten der Universität. Hierbei verwendeten sie ein eigenes, internetfähiges Gerät (Laptop oder Tablet), indem sie die Umfrage über einen Browser öffneten und im Rahmen dieser Sitzung vollständig bearbeiteten. Bei Verständnis- und Rückfragen stand somit jederzeit ein Studienmitarbeiter zur Verfügung. Für die Bearbeitung des Fragebogens benötigten die Studienteilnehmer durchschnittlich 2 Stunden und 41 min.

\subsubsection{Studienzeitraum}

Die Datenerhebungen fanden während der vorlesungsfreien Zeit jeweils in den Sommersemestern 2017 und 2018 statt, maximal drei Wochen nach Absolvieren des Blockpraktikums Chirurgie.

\subsubsection{Rekrutierung der Studienteilnehmer}

Die Rekrutierung der Studienteilnehmer erfolgte in einer Informationsveranstaltung zu Beginn des 3-wöchigen Blockpraktikums im Rahmen der sogenannten „TpFWoche" (TpF=Training praktischer Fähigkeiten). ${ }^{85}$ Hierbei wurden die Studierenden von einem ärztlichen Mitarbeiter ausführlich über die Studie informiert. Die 
Teilnahme an der Studie war freiwillig. Die Studieneinwilligung erfolgte schriftlich und konnte jederzeit widerrufen werden. Die Studienteilnehmer erhielten eine finanzielle Aufwendung in Höhe von 5 Zeitstunden entsprechend des Tarifs als wissenschaftliche Hilfskraft.

\subsubsection{Lehrkrankenhäuser und Zuteilung}

Zuteilung der Studierenden zu den jeweiligen Lehrkrankenhäusern erfolgt im Rahmen der Zentralen Eintragung zu den Semesterveranstaltungen über das Verwaltungsprogramm Fact-Science. Hierbei kann der Studierende im Vorfeld über ein Punktesystem Prioritäten bezüglich des Zeitraums und des Lehrkrankenhauses angeben. Bei Überbelegung wird über die Einteilung per Losverfahren entschieden. In der Auswertung bezüglich der Krankenhauswahl erfolgte eine Zuteilung der einzelnen Häuser in Lehrkrankenhäuser vs. Uniklinikum.

Zur Überprüfung der erreichten Lernziele in Bezug auf die Krankenhäuser haben wir folgende Kategorien eingeteilt: Studierende, die ihr Blockpraktikum Chirurgie an dem Universitätsklinikum absolviert haben, an akademischen Lehrkrankenhäusern mit >200 chirurgischen Betten, Lehrkrankenhäuser mit $100-200$ Bettenplätzen in allen chirurgischen Abteilungen und solche mit unter 100 Betten (siehe Leistungsreport der hessischen Krankenhäuser 2017). ${ }^{86}$

Über 200 Bettenplätze in der Chirurgie halten die BG Unfallklinik Frankfurt, das Sana Klinikum Offenbach, das Agaplesion Markus Krankenhaus Frankfurt und das Klinikum Höchst vor. 100 bis 200 chirurgische Bettenplätze stehen im Klinikum Darmstadt, im Klinikum Hanau, in den Main-Kinzig Kliniken Gelnhausen, den MainTaunus Kliniken Bad Soden, der Asklepios Klinik in Langen, dem Krankenhaus Nordwest in Frankfurt und den Hochtaunuskliniken zur Verfügung. Weniger als 100 Bettenplätze sind in den chirurgischen Abteilungen des Hospitals zum Heiligen Geist in Frankfurt, des St. Katharinen-Krankenhaus Frankfurt, des Bürgerhospital Frankfurt, in denen der Asklepios Klinik Seligenstadt, des St. Elisabethen Krankenhauses Frankfurt, des Krankenhaus Sachsenhausen und des Ketteler Krankenhaus Offenbach vorhanden (Tab. 4). 


\begin{tabular}{|c|c|c|}
\hline Krankenhaus & Kategorie & $\begin{array}{l}\text { Anzahl Bettenplätze Chirurgie gesamt (It. } \\
\text { Versorgungsatlas Hessen } 2017 \text { "Chirurgie", } \\
\text { "Herzchirurgie", "Neurochirurgie", "MKPG") }\end{array}$ \\
\hline BG Unfallklinik & $>200$ & 360 \\
\hline Uniklinikum Frankfurt & $>200$ & 328 \\
\hline Sana Klink Offenbach & $>200$ & 279 \\
\hline \multicolumn{3}{|l|}{ Agaplesion Markus } \\
\hline Krankenhaus & $>200$ & 233 \\
\hline Klinikum Höchst & $>200$ & 211 \\
\hline Klinikum Darmstadt & $100-200$ & 167 \\
\hline Klinikum Hanau & $100-200$ & 149 \\
\hline $\begin{array}{l}\text { Main-Kinzig Kliniken } \\
\text { Gelnhausen }\end{array}$ & $100-200$ & 138 \\
\hline Main-Taunus Kliniken & & \\
\hline Bad Soden & $100-200$ & 138 \\
\hline Asklepios Klinik Langen & $100-200$ & 125 \\
\hline Krankenhaus Nordwest & & \\
\hline Frankfurt & $100-200$ & 122 \\
\hline Hochtaunus-Kliniken & $100-200$ & 119 \\
\hline $\begin{array}{l}\text { Hospital zum Heiligen } \\
\text { Geist Frankfurt }\end{array}$ & $<100$ & 82 \\
\hline $\begin{array}{l}\text { St. Katharinen- } \\
\text { Krankenhaus Frankfurt }\end{array}$ & $<100$ & 80 \\
\hline Bürgerhospital Frankfurt & $<100$ & 80 \\
\hline Asklepios Klinik & & \\
\hline Seligenstadt & $<100$ & 75 \\
\hline St. Elisabethen & & \\
\hline Krankenhaus Frankfurt & $<100$ & 71 \\
\hline Krankenhaus & & \\
\hline Sachsenhausen & $<100$ & 64 \\
\hline $\begin{array}{l}\text { Ketteler Krankenhaus } \\
\text { Offenbach }\end{array}$ & $<100$ & 61 \\
\hline
\end{tabular}

Tab. 4 Auflistung der Lehrkrankenhäuser und deren Anzahl chirurgischer Bettenplätze It.

Versorgungsatlas Hessen 2017

\subsubsection{Fachrichtungen}

Im Rahmen des Blockpraktikums Chirurgie werden die Studierenden an den jeweiligen Krankenhäusern und auch an der Universitätsklinik unterschiedlichen chirurgischen Fachrichtungen zugeordnet. Sie werden in den Arbeitsalltag der Station integriert und nehmen an den Operationen der jeweiligen Abteilung teil. An manchen Kliniken besuchen die Studierende zusätzlich die Notfallambulanz oder rotieren zwischen den Stationen und Operationssälen unterschiedlicher 
Fachrichtungen. Wir haben uns aus Gründen der Vergleichbarkeit und Übersicht dazu entschieden, die Auswertung in folgenden Kategorien zusammenzufassen:

- „Unfallchirurgie“ (UCH),

- „Allgemeinchirurgie“ (ACH),

- „Rotiert“ und

- „Rest“.

Unter „Rotiert“ verstehen sich die Studierenden, die innerhalb der zwei Wochen Blockpraktikum Stationen unterschiedlicher Fachrichtungen besucht haben oder zusätzlich in der Notfallaufnahme Kontakt mit diversen chirurgischen Fachrichtungen hatten. In die Kategorie „Rest“ zählen wir Studierende, die ausschließlich die kleineren chirurgischen Fachrichtungen im Rahmen ihres Blockpraktikums besucht haben. Im Konkreten sind hierunter die Fächer Kinderchirurgie, Gefäßchirurgie, Thoraxchirurgie, Plastische Chirurgie, MundKiefer-Gesichtschirurgie und Adipositaschirurgie besucht worden.

\subsubsection{Inhalte des Blockpraktikums Chirurgie}

Im Rahmen des Blockpraktikums sind Inhalte eines Testathefts abzuarbeiten und von dem jeweiligen Betreuer in der Klinik gegenzeichnen zu lassen. Ebenfalls ist die Abgabe eines Portfolios verpflichtend. Im Anhang befinden sich das Testatheft und die Vorgabekriterien für das Portfolio inklusive eines Beispielportfolios. Die Ausgestaltung der Inhalte auf den jeweiligen Stationen obliegt den Unterrichtsbeauftragten der Uniklinik bzw. des Lehrkrankenhauses und richtet sich nach dem chirurgischen Spektrum der Abteilung.

\subsection{Statistische Auswertung}

Die epidemiologischen Daten, sowie Mittelwerte (MW), Standardabweichungen (SD), Minima (Min) und Maxima (Max) wurden mit Excel ausgewertet. Die vertiefte statistische Auswertung erfolgte mittels Bias. Hier wurden zur Ermittlung der p-Werte zwei unterschiedliche nicht-parametrische Tests angewendet. Die Auswertung bei zwei Populationen erfolgte mit dem Wilcoxon-Mann-Whitney-Test und für 4 Populationen mit dem Kruskal-Wallis-Test. Zur Korrektur der Alpha Fehler bei 
multiplen Vergleichen ist in einem Post-Hoc Test nach der Bonferroni Methode durchgeführt worden. 


\section{Ergebnisse}

\subsection{Teilnehmer}

In den Sommer-Semestern 2017 nahmen 153 und 2018162 Studierende am Blockpraktikum Chirurgie teil. In der vorliegenden Arbeit konnten insgesamt 81 Studierende eingeschlossen werden, davon 53 Frauen und 28 Männer (Abb. 6). Dies entspricht der Geschlechterverteilung der Medizinstudierenden an der Goethe Universität Frankfurt. Im Schnitt waren die Studierenden 22,89 Jahre alt. 7 Personen gaben an, vor dem Medizinstudium eine weitere Ausbildung/Studium absolviert zu haben, 2 davon in medizinischen Bereichen. Die Bearbeitung des OnlineFragekatalogs dauerte im Mittel 2 Stunden und $41 \mathrm{~min}$. Die Bearbeitung der Kapitel $5-20$ erfolgte von 81 Studierenden, das Kapitel 21 bearbeiteten nur 79 Personen.

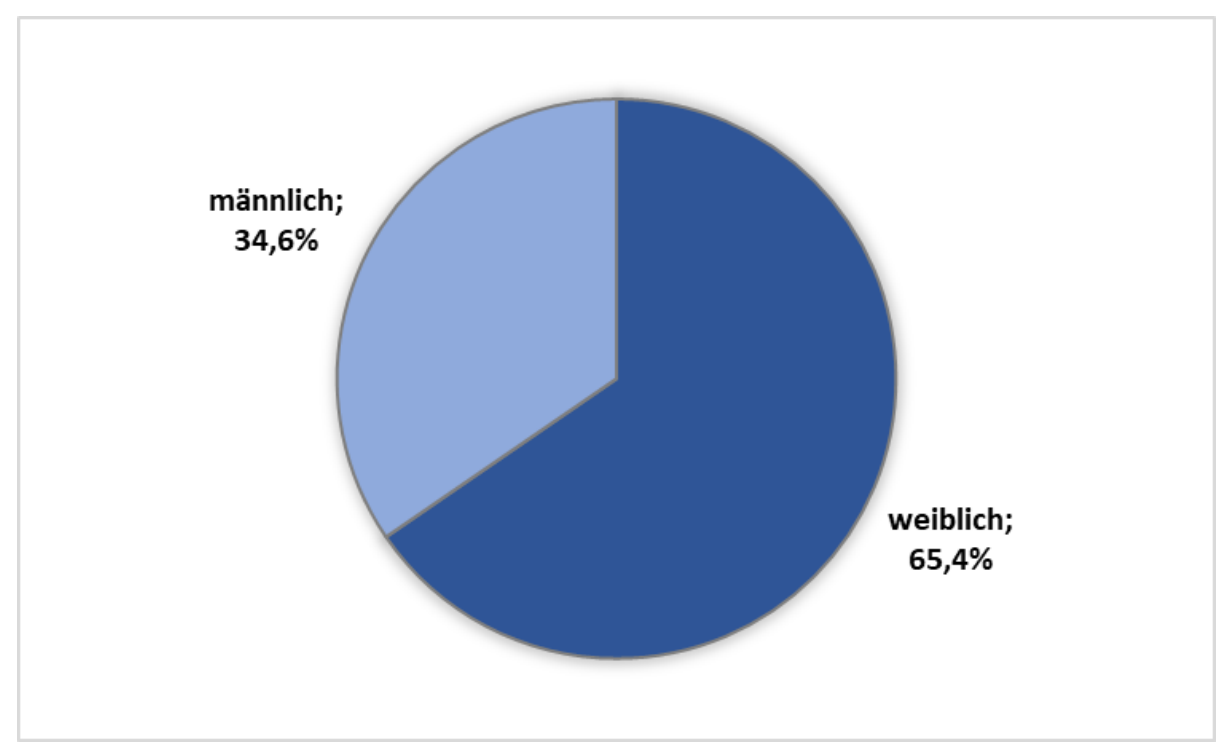

Abb. 6 Geschlechterverteilung der Studienteilnehmenden;

Verteilung dargestellt in \% mit dunkelblau = weibliche und hellblau = männliche Teilnehmende.

\subsection{Fachrichtungen}

Gemäß der Einteilung in die verschiedenen Fachrichtungen absolvierten 21 Studierende ihr Blockpraktikum im Fachbereich ACH und 22 im Fachbereich $\mathrm{UCH}$. 27 Studierende rotierten im Rahmen ihres Blockpraktikums und 11 besuchten „sonstige“ Fachrichtungen (Abb. 7). 


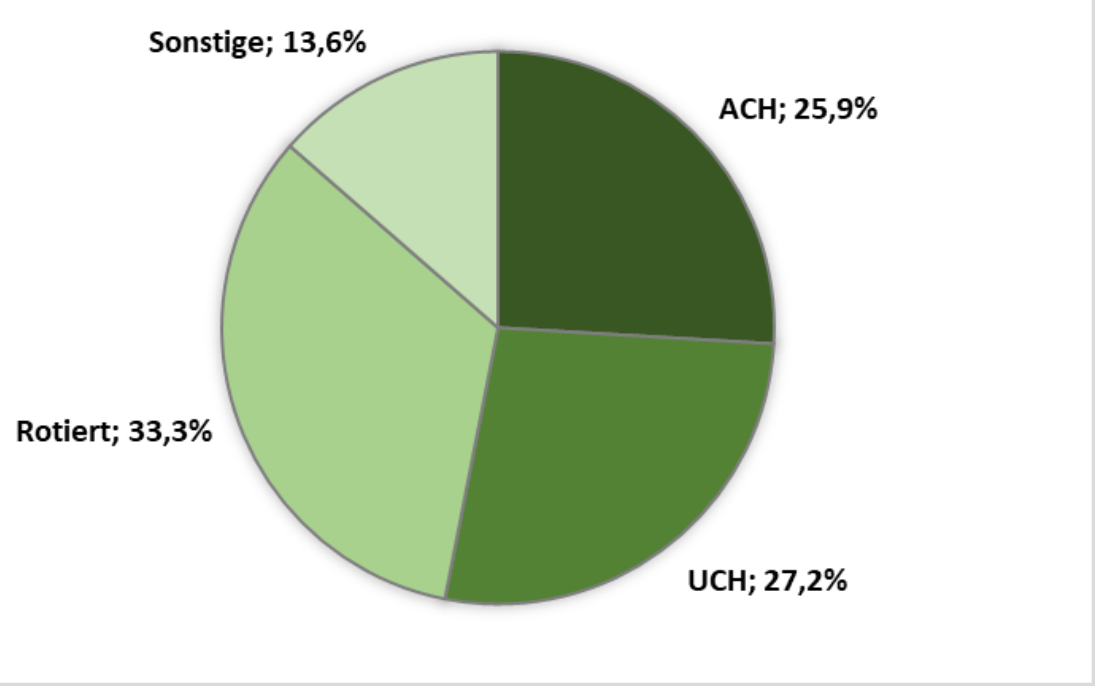

Abb. 7 Verteilung der besuchten Fachrichtungen; Dargestellt in \% der Teilnehmenden. $\mathrm{UCH}=$ Unfallchirurgie, $\mathrm{ACH}=$ Allgemeinchirurgie, Rotiert = Rotation durch versch. chirurg. Fachgebiete, Sonstige $=$ andere chirurg. Fachgebiete.

\subsection{Lehrkrankenhäuser + Uniklinikum}

Die Studienteilnehmer absolvierten ihr Blockpraktikum insgesamt an 17 unterschiedlichen (Lehr-) Krankenhäusern.

Von den insgesamt 81 Studienteilnehmern absolvierten 21 Studierende ihr Blockpraktikum Chirurgie an der Universitätsklinik Frankfurt, 23 Studierende in einem Krankenhaus der Kategorie >200 Betten, 13 Studierende an Kliniken mit 100 - 200 chirurgischen Betten und 24 Studierende an Kliniken mit unter 100 Bettenplätzen in der Chirurgie. (Abb. 8) 


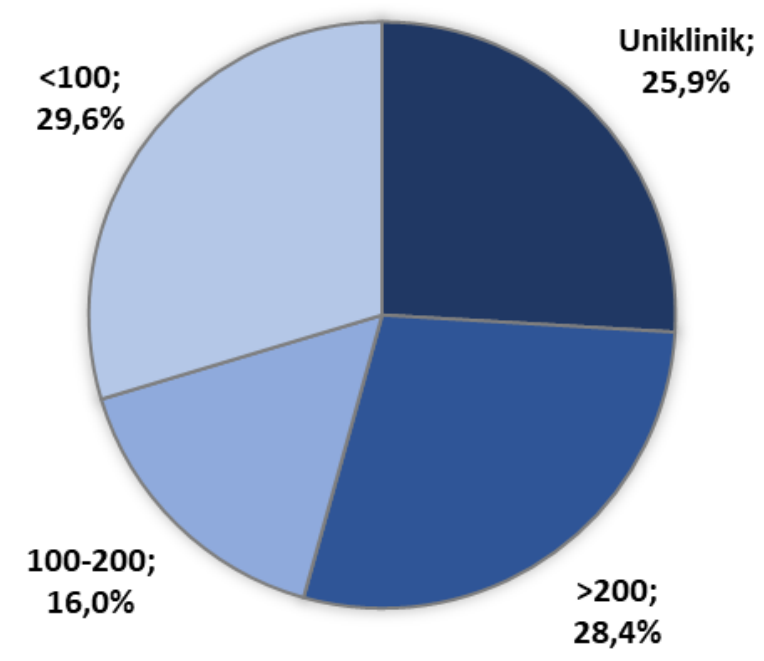

Abb. 8 Verteilung der Studierenden auf die einzelnen (Lehr-)Krankenhäuser; Dargestellt in \% der Teilnehmenden. $>200,100-200,<100$ bezieht sich auf die Anzahl der chirurgischen Betten.

\subsection{Lernziele}

\subsubsection{Gesamt}

Insgesamt konnten wir zeigen, dass das zweiwöchige BP Chirurgie im Mittel 8,78 $\pm 5,1 \%$ (Min. = 1,01\%; Max. = 29,84\%) aller im NKLM festgehaltenen Lernziele abdecken kann. (Abb. 9)

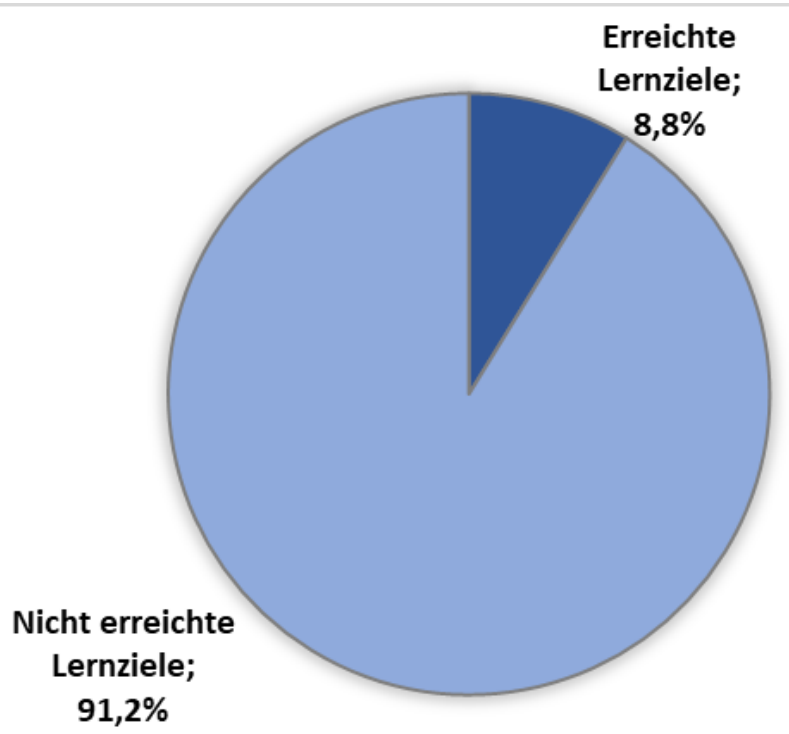

Abb. 9 Verteilung der im Blockpraktikum erreichten Lernziele; Dargestellt in $\%$ hellblau $=$ nicht erreicht; dunkelblau = erreichte Lernziele. 


\subsubsection{Abschnitte 1 - 3 und Kapitel}

Unsere Auswertungen zeigen, dass den Studierenden im zweiwöchigen Blockpraktikum Chirurgie mit 29,92 $\pm 15,22 \%$ (Min. $=0 \%$; Max. $=63,10 \%$ ) fast ein Drittel der im NKLM festgehaltenen Lernziele der „Rollen der Ärztin/des Arztes“ vermittelt werden können. Die Abdeckung der Lernziele aus Abschnitt 2 zu Physiologie/Pathophysiologie, klinisch-praktischer

Fertigkeiten, therapeutischen/diagnostischen Verfahren und Notfallmanagement, kann im Rahmen des Blockpraktikum deutlich geringer vorgenommen werden und liegt bei 6,88 $\pm 4,99 \%$ (Min. $=0 \%$; Max. $=30,10 \%$ ). Im Abschnitt 3 Anlässe ärztlicher Konsultation und einzelnen Krankheitsbilder ist die Abdeckung mit 6,29 $\pm 6,58 \%$ (Min. $=0 \% ;$ Max. $=51,61 \%)$ noch geringer. $($ Abb. 10$)$

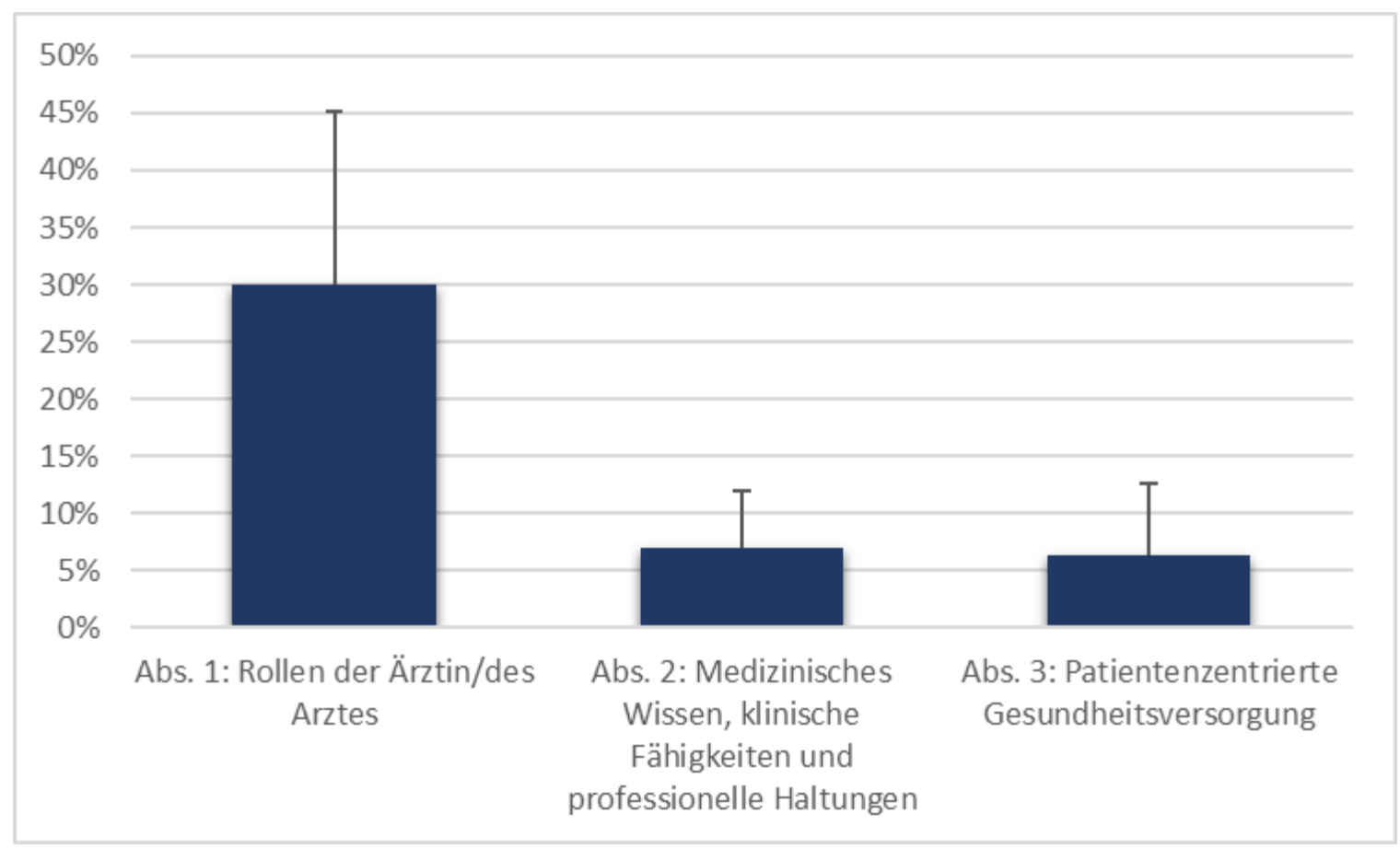

Abb. 10 Insgesamt erreichte Lernziele der Abschnitte 1, 2 und 3 des NKLM; Mittelwerte + Standardabweichungen dargestellt in \%.

In Abschnitt 1 der ärztlichen Rollen wurde eine Abdeckung der im NKLM formulierten Lernziele zwischen $16-50 \%$ angegeben. Besonders hervorzuheben sind hier die Kapitel 5 „Der Arzt als medizinischer Experte“ mit 45,78 $\pm 22,00 \%$ (Min. = 0,00\%; 
Max. $=91,67 \%$ ) und Kapitel 7 „Der Arzt als Kommunikator“ 47,94 $\pm 28,01 \%$ (Min. = 0,00\%; Max. $=100,00 \%)$. (Abb. 11).

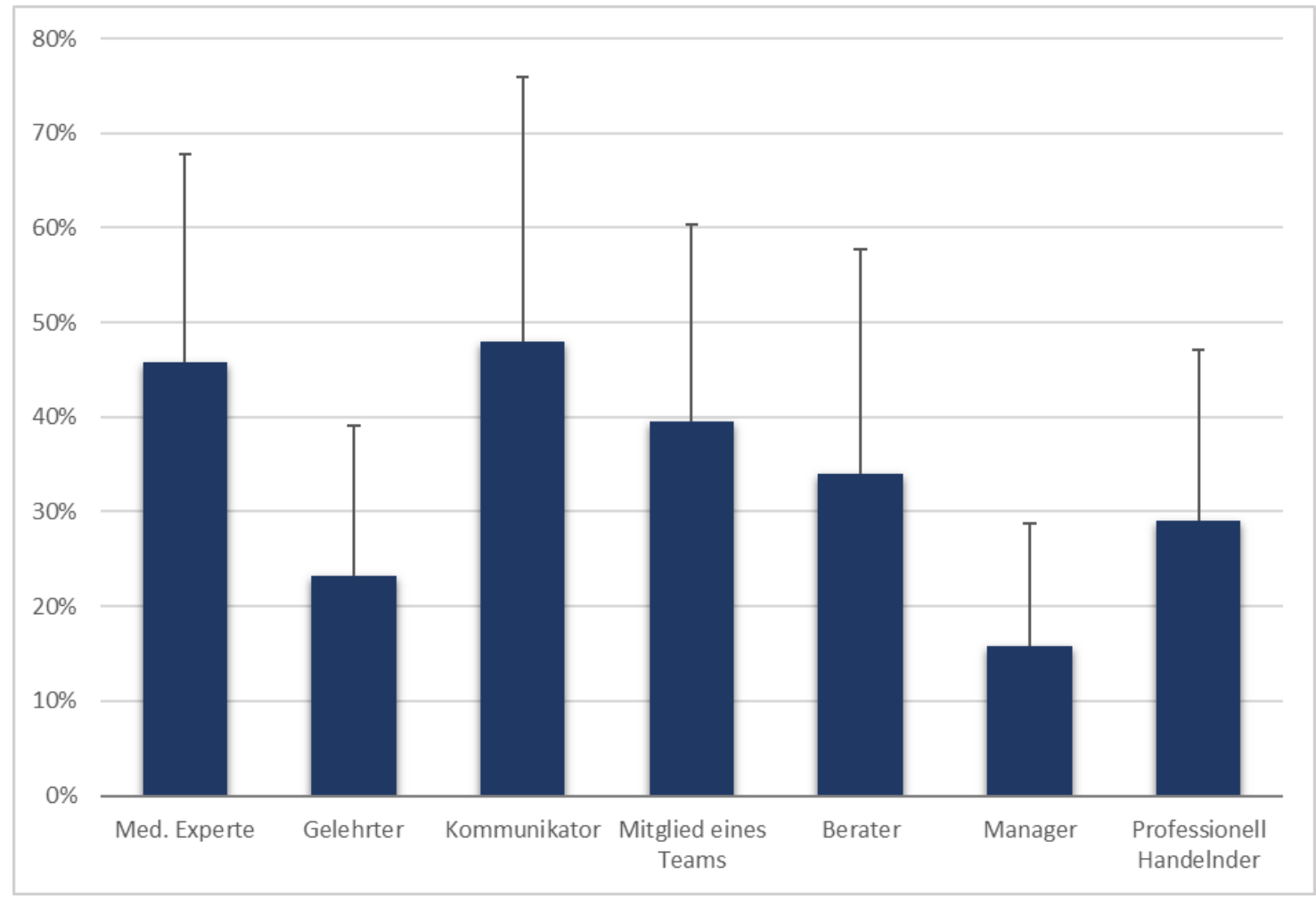

Abb. 11 Insgesamt erreichte Lernziele der Kapitel 5-11 in Abschnitt 1; Mittelwerte \pm Standardabweichung dargestellt in \%.

\begin{tabular}{|lcccc|}
\hline Kapitel „Der Arzt als...“ & MW & (SD & Min. & Max. \\
\hline 5 Medizinischer Experte & 45,78 & 22,00 & 0,00 & 91,67 \\
6 Gelehrter & 23,26 & 15,82 & 0,00 & 67,74 \\
7 Kommunikator & 47,94 & 28,01 & 0,00 & 100,00 \\
8 Mitglied eines Teams & 39,52 & 20,78 & 0,00 & 78,26 \\
9 Gesundheitsberater-/fürsprecher & 34,01 & 23,72 & 0,00 & 94,44 \\
10 Verantwortungsträger und & & & & \\
Manager & 15,85 & 12,91 & 0,00 & 54,05 \\
11 Professionell Handelnder & 28,99 & 18,05 & 0,00 & 81,25 \\
\hline
\end{tabular}

Tab. 5 Erreichte Lernziele des Abschnitts 1 des NKLM. Mittelwerte \pm Standardabweichungen, Minima und Maxima der Mittelwerte dargestellt in \%.

Die erreichten Lernziele der „ärztlichen Rollen“ sind zu 77,23 $\pm 3,80 \%$ (Min. = 69,18\%; Max. $=82,36 \%$ ) implizit an die Studierenden vermittelt worden. (Abb. 12) 


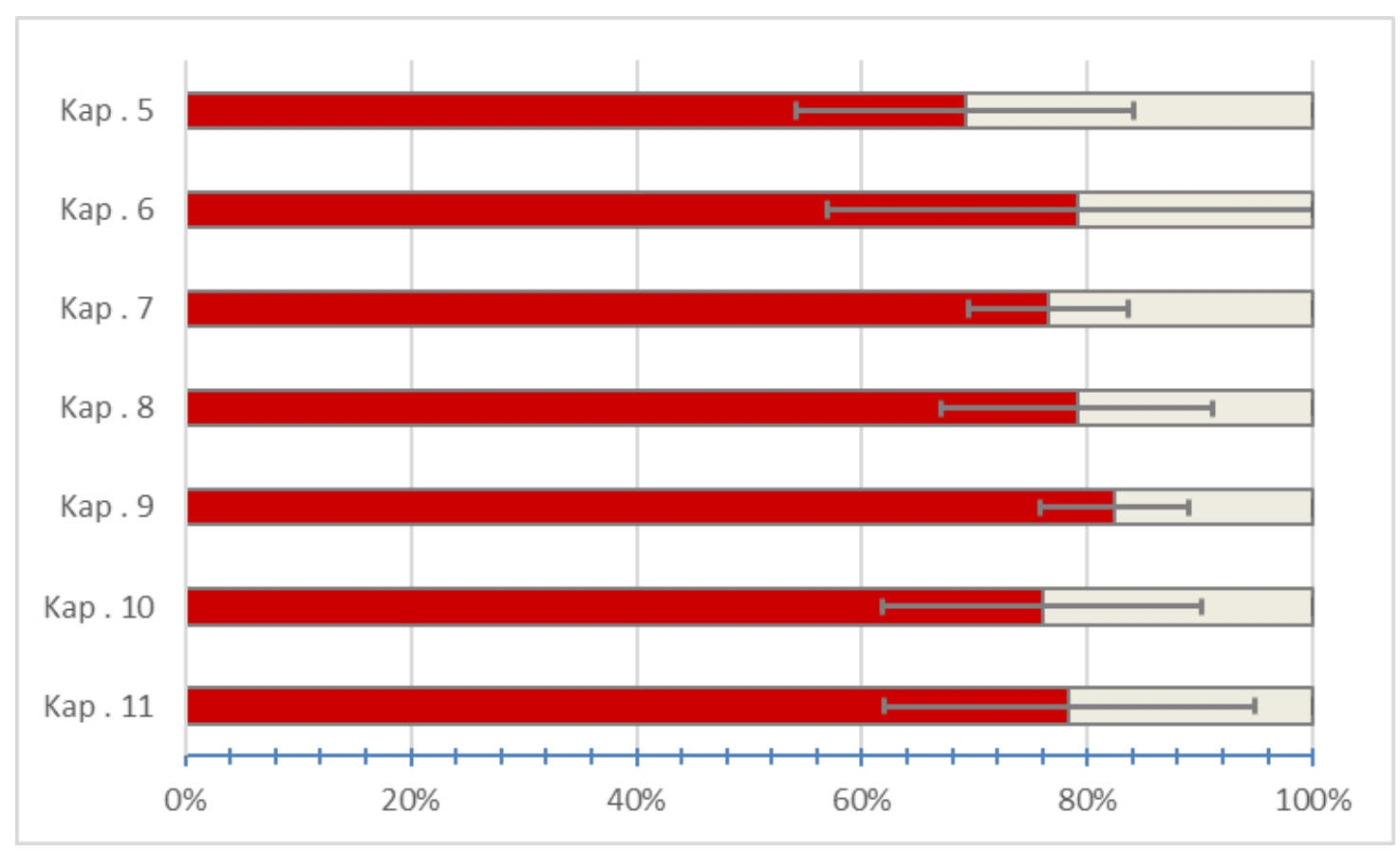

Abb. 12 Implizit vermittelte Lernziele in Abschnitt 1 des NKLM; Mittelwerte \pm Standardabweichungen dargestellt in \%; rot = implizit; hellgrau = explizit.

\begin{tabular}{|lcccc|}
\hline Implizit vermittelte Lernziele & MW & SD & min & max \\
\hline 5 Medizinischer Experte & 69,18 & 16,39 & 40,00 & 100,00 \\
6 Gelehrter & 79,08 & 14,14 & 50,00 & 100,00 \\
7 Kommunikator & 76,51 & 6,63 & 64,71 & 83,33 \\
8 Mitglied eines Teams & 79,08 & 12,05 & 54,17 & 100,00 \\
9 Gesundheitsberater/-fürsprecher & 82,36 & 7,13 & 67,16 & 100,00 \\
10 Verantwortungsträger und & & & & \\
Manager & 76,00 & 22,18 & 0,00 & 100,00 \\
11 Professionell Handelnder & 78,38 & 14,95 & 43,59 & 100,00 \\
\hline
\end{tabular}

Tab. 6 Implizit vermittelte Lernziele des Abschnitt 1; Mittelwerte \pm Standardabweichungen, Minima und Maxima dargestellt in \%.

Über den Abschnitt $2 \mathrm{zu}$ medizinischem Wissen, klinischen Fähigkeiten und professionellen Haltungen zeigte sich eine heterogene Verteilung der erreichten Lernziele. Besonders hervorzuheben sind die Kapitel 14b „Klinisch praktische Fertigkeiten“ und Kapitel 14c „Ärztliche Gesprächsführung“. Die darin festgehaltenen Lernziele kann das BP Chirurgie zu 15,49 $\pm 7,78 \%$ (Min. = 0,00\%; Max. $=41,30 \%$ ) bzw. 22,98 $\pm 16,47 \%$ (Min. = 0,00\%; Max. $=70,69 \%)$ abdecken, 
wohingegen in den restlichen Kapiteln des Abschnitts 2 nur eine Abdeckung von um ca. $5 \%$ stattfinden konnte (Abb. 13).

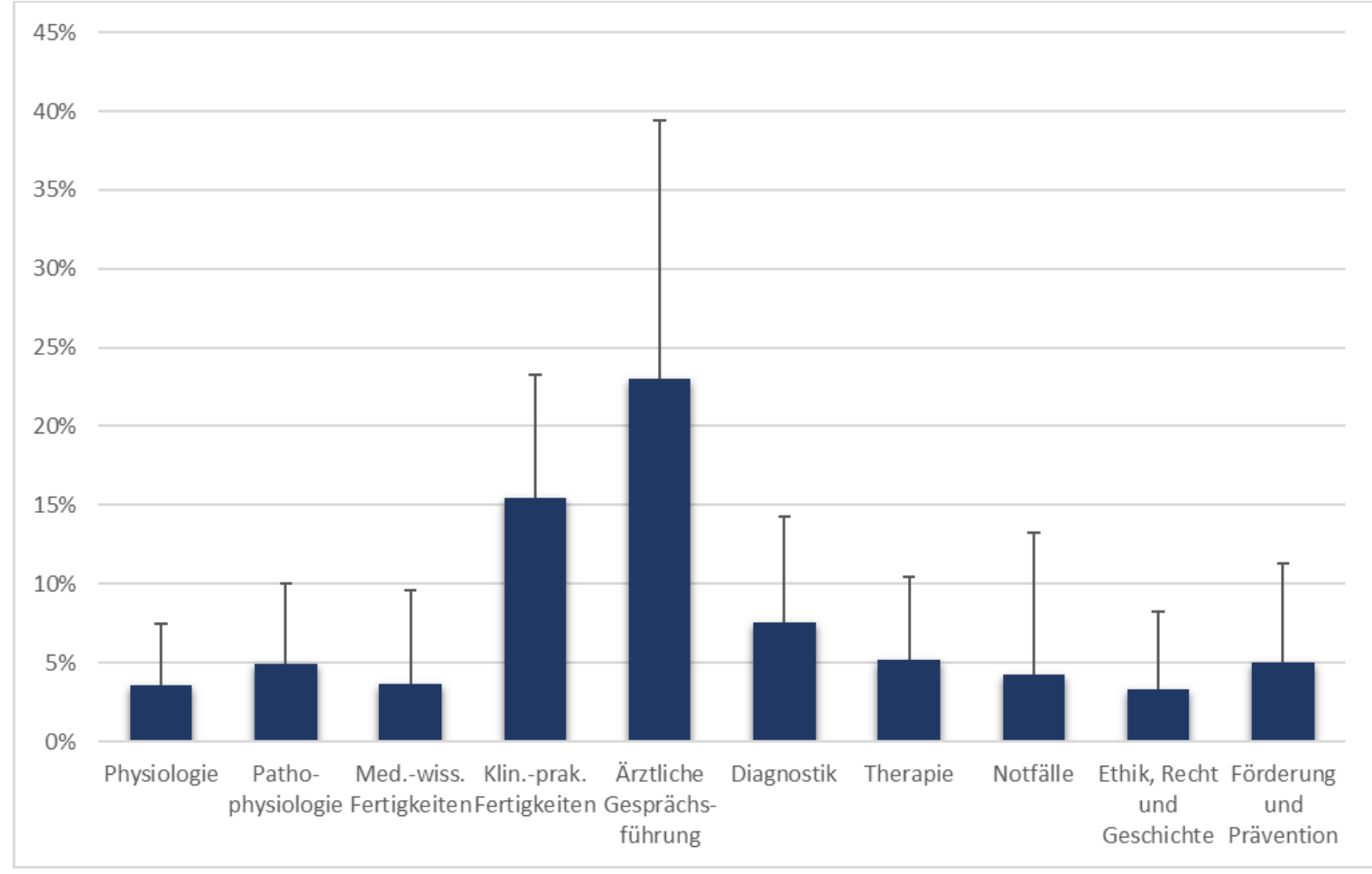

Abb. 13 Erreichte Lernziele der Kapitel 12 - 19 in Abschnitt 2; Mittelwerte + Standardabweichungen dargestellt in \%.

\begin{tabular}{|lcccc|}
\hline Kapitel & MW & SD & Min. & Max. \\
\hline 12 Physiologie & 3,56 & 3,89 & 0,00 & 21,41 \\
13 Pathophysiologie & 4,93 & 5,10 & 0,00 & 23,49 \\
14a Medizinisch-wissenschaftliche & & & & \\
Fertigkeiten & 3,68 & 5,92 & 0,00 & 24,32 \\
14b Klinisch-praktische Fertigkeiten & 15,49 & 7,78 & 0,00 & 41,30 \\
14c Ärztliche Gesprächsführung & 22,98 & 16,47 & 0,00 & 70,69 \\
15 Diagnostik & 7,60 & 6,68 & 0,00 & 31,87 \\
16 Therapie & 5,19 & 5,24 & 0,00 & 30,80 \\
17 Notfälle & 4,25 & 8,96 & 0,00 & 66,67 \\
18 Ethik, Recht und Geschichte der Medizin & 3,30 & 4,90 & 0,00 & 27,14 \\
19 Gesundheitsförderung und Prävention & 5,04 & 6,22 & 0,00 & 27,38 \\
\hline
\end{tabular}

Tab. 7 Erreichte Lernziele des Abschnitts 2 des NKLM; Mittelwerte \pm Standardabweichungen, Minima und Maxima der Mittelwerte dargestellt in \%. 
In Abschnitt 3 zeigt sich, dass das BP Chirurgie in Bezug auf Kapitel 20 10,81\% (SD $=9,89 \%$; Min. $=0 \%$; Max. $=51,61 \%)$ der Anlässe für ärztliche Konsultationen abdecken kann. Die Lernziele aus Kapitel 21 „Erkrankungsbezogene Prävention, Diagnostik, Therapie, Versorgungs- und Notfallmanagement" können relativ gering zu 4,50 $\pm 3,46 \%$ (Min. = 0,23\%; Max. = 16,78\%) im BP Chirurgie vermittelt werden (Abb. 14)

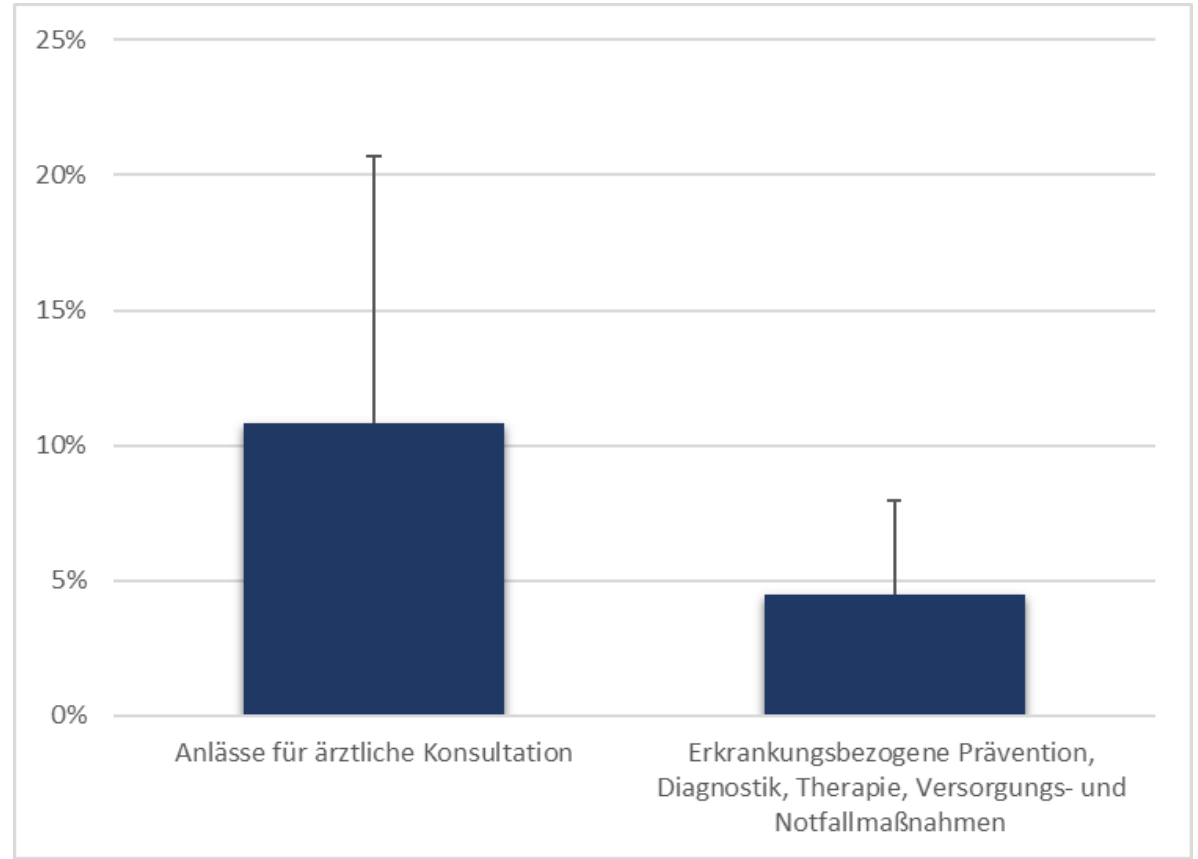

Abb. 14 Erreichte Lernziele der Kapitel 20 und 21 in Abschnitt 3; Mittelwerte + Standardabweichungen dargestellt in \%.

Trotz der insgesamt geringen Abdeckung zeigt sich innerhalb der 11 Unterteilungen in Kapitel 21 eine unterschiedliche Auslastung. Der NKLM listet in Kapitel 21 relevante Erkrankungsbilder auf und teilt diese nach Primären Organsystemen ein. Die Lernziele zu den Abschnitten der Primären Organsysteme „Kardiovaskulär“, "Muskuloskelettal und Weichgewebe" und "Verdauungssysteme" werden anteilig deutlich mehr im Blockpraktikum Chirurgie vermittelt als Lernziele anderer Unterkapitel des Kapitels 21 (Abb. 15). 


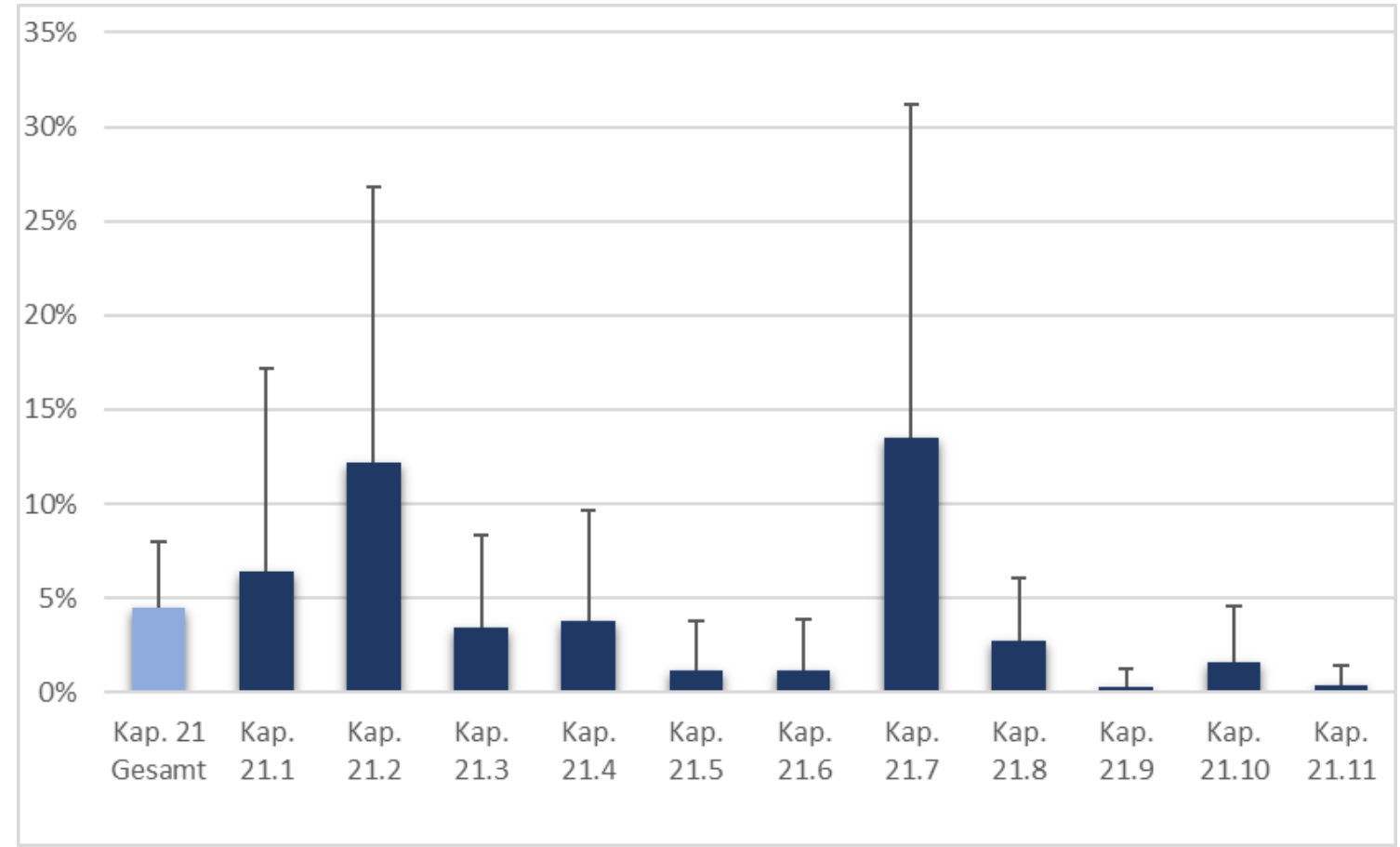

Abb. 15 Erreichte Lernziele der Unterkapitel des Kapitel 21 zu Primären Organsystemen.

Mittelwerte + Standardabweichungen dargestellt in \%.

\begin{tabular}{|lcccc|}
\hline Unterkapitel & MW & SD & Min. & Max. \\
\hline 21.1 Kardiovaskuläres System & 6,44 & 10,69 & 0,00 & 65,52 \\
21.2 Muskuloskelettales System & 12,17 & 14,66 & 0,00 & 62,50 \\
21.3 Hormone und & & & & \\
Stoffwechselsystem & 3,46 & 4,88 & 0,00 & 20,00 \\
21.4 Respiratorisches System & 3,75 & 5,91 & 0,00 & 29,41 \\
21.5 Blut und Immunologie & 1,13 & 2,66 & 0,00 & 9,52 \\
21.6 Urogenitales System & 1,14 & 2,72 & 0,00 & 13,64 \\
21.7 Verdauungssystem & 13,51 & 17,70 & 0,00 & 81,25 \\
21.8 Sinnesystem & 2,69 & 3,41 & 0,00 & 15,56 \\
21.9 Haut & 0,27 & 0,93 & 0,00 & 3,45 \\
21.10 Nervensystem & 1,56 & 3,04 & 0,00 & 13,51 \\
21.11 Schwangerschaft, Fetal-, & & & & \\
Perinatal- und Neonatalzeit & 0,34 & 1,05 & 0,00 & 5,13 \\
\hline
\end{tabular}

Tab. 8 Erreichte Lernziele der Unterkapitel des Kapitel 21 zu Primären Organsystemen; Mittelwerte \pm Standardabweichungen, Minima und Maxima der Mittelwerte dargestellt in \%. 


\subsection{3 männlich/weiblich}

Im Gesamten gaben Frauen an 8,22 $\pm 5,11 \%$ (Min. $=1,90 \%$; Max. $=29,84 \%)$ der NKLM Lernziele im Blockpraktikum Chirurgie erreicht zu haben. Im Vergleich dazu lag der Anteil erreichter Lernziele bei den männlichen Studienteilnehmern bei 9,84 4,90\%; Min. = 1,01\%; Max. = 20,91\%) (Abb. 16).

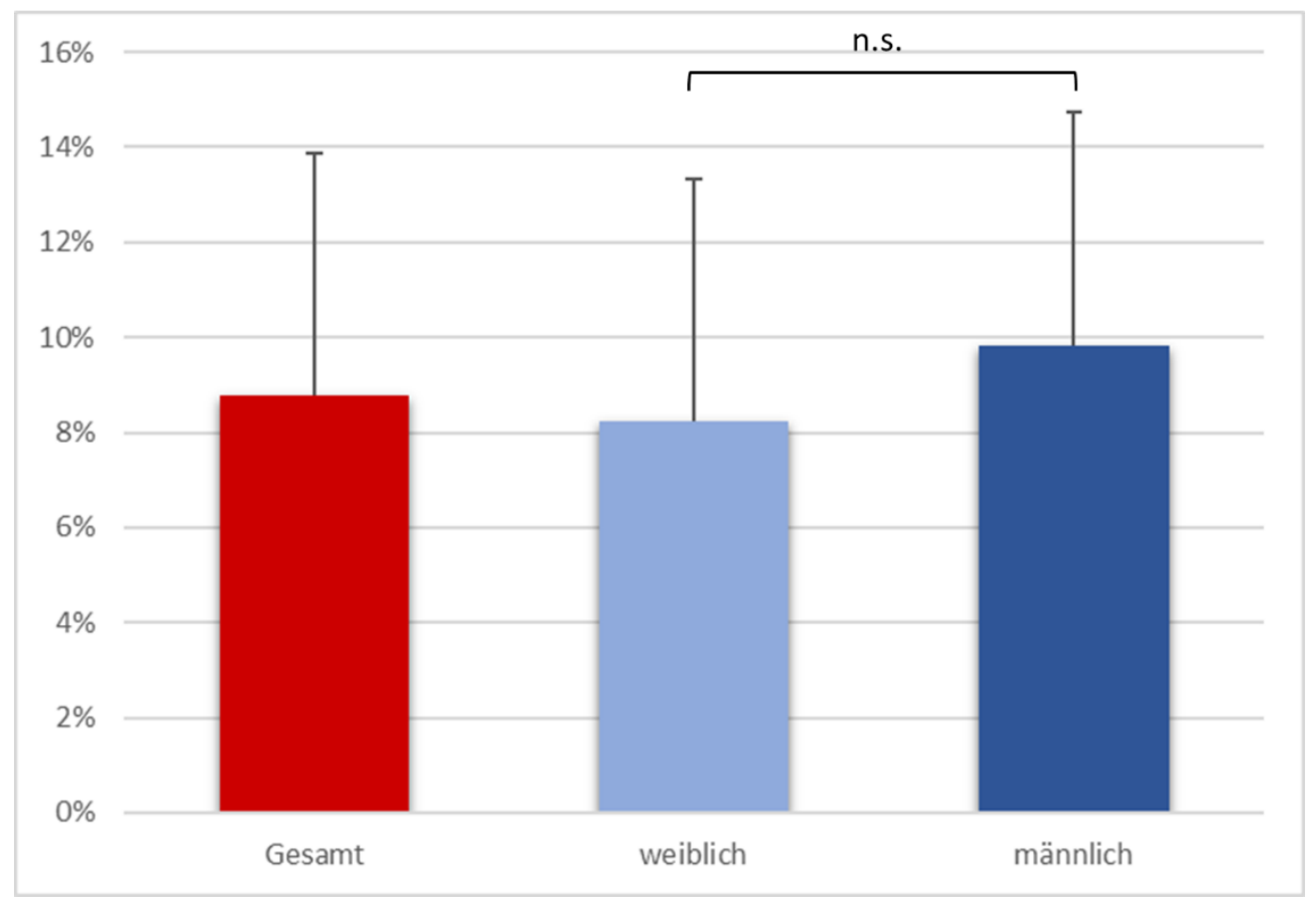

Abb. 16 Insgesamt erreichte Lernziele nach Geschlecht; Mittelwerte + Standardabweichungen dargestellt in \%; rot $=$ gesamt, hellblau $=$ weiblich, dunkelblau $=$ männlich; ${ }^{* *} p<0.01,{ }^{*} p<0.05$, n.s. $p>0.05$.

In Abschnitt 1 der ärztlichen Rollen zeigt sich deutlich, dass Männer angeben mehr Lernziele vermittelt zu bekommen als Frauen (Abb. 17). 


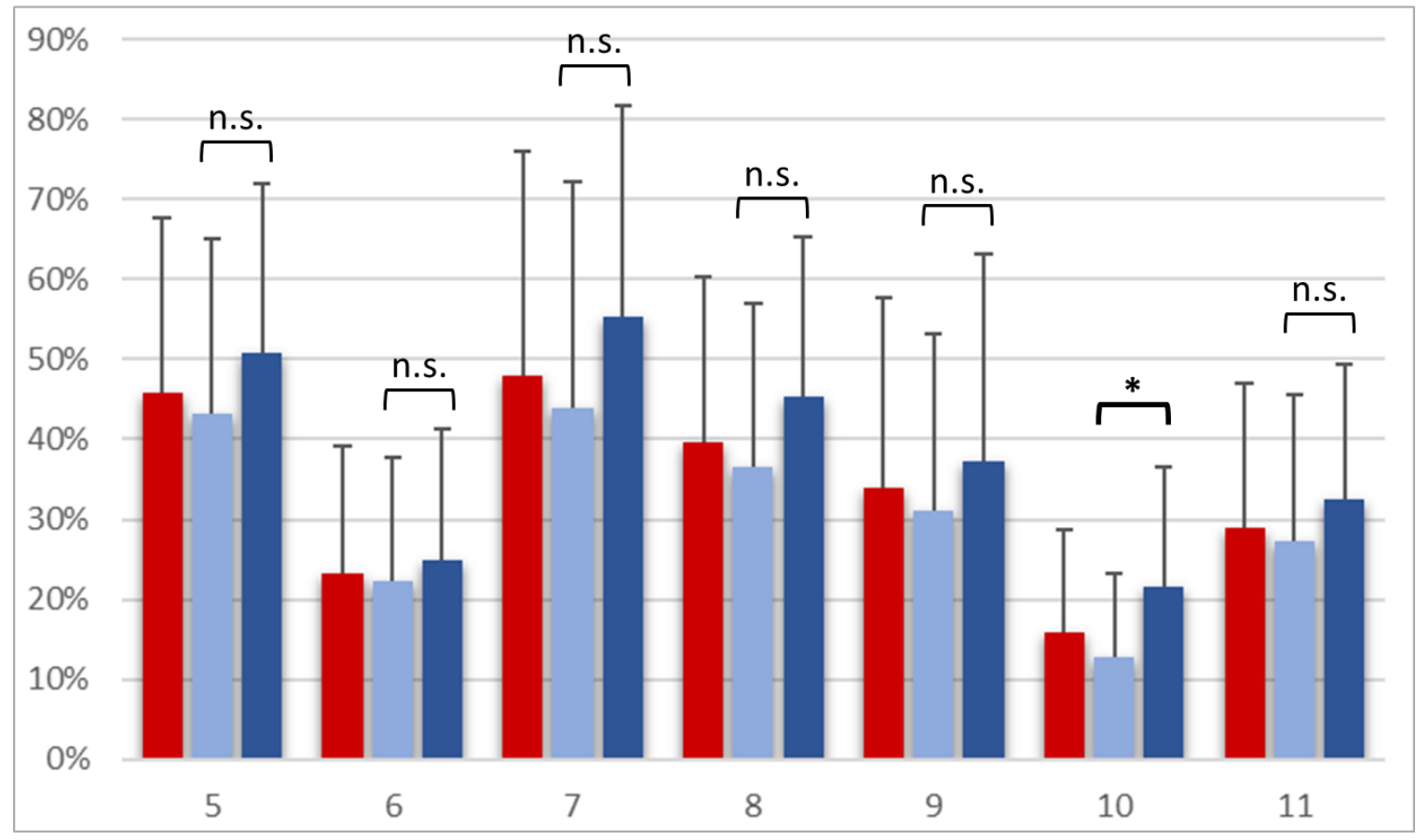

Abb. 17 Erreichte Lernziele in Abschnitt 1 nach Geschlecht; Mittelwerte + Standardabweichungen dargestellt in \%; rot = gesamt; hellblau = weiblich, dunkelblau = männlich. ${ }^{* *} p<0.01,{ }^{*} p<0.05={ }^{*}$, n.s. $p>0.05$.

\begin{tabular}{|c|c|c|c|c|c|c|c|c|c|}
\hline \multirow[t]{2}{*}{ Kapitel } & \multicolumn{4}{|c|}{ Frauen } & \multicolumn{5}{|c|}{ Männer } \\
\hline & MW & SD & $\min$ & $\max$ & MW & SD & $\min$ & $\max$ & $\mathbf{P}$ \\
\hline 5 Medizinischer Expert & 43,16 & 21,95 & 0 & 79,17 & 50,74 & 21,25 & 8,33 & 91,67 & 0.179558 \\
\hline 6 Gelehrter & 22,40 & 15,41 & 0 & 51,61 & 24,88 & 16,45 & 0 & 67,74 & 0.682078 \\
\hline 7 Kommunikator & 44,03 & 28,06 & 0 & 100,0 & 55,36 & 26,37 & 0 & 100,0 & 0.106896 \\
\hline 8 Mitglied eines Teams & 36,48 & 20,47 & 0 & 78,26 & 45,27 & 20,12 & 13,04 & 78,26 & 0.130517 \\
\hline $\begin{array}{l}9 \text { Gesundheitsberater- } \\
\text { /Fürsprecher }\end{array}$ & 31,13 & 21,97 & 0 & 83,33 & 37,30 & 25,81 & 5,56 & 88,89 & 0.310782 \\
\hline $\begin{array}{l}10 \\
\text { Verantwortungsträger } \\
\text { und Manager }\end{array}$ & 12,85 & 10,50 & 0 & 35,14 & 21,53 & 14,97 & 2,70 & 54,05 & 0.010883 \\
\hline $\begin{array}{l}11 \text { Professionell } \\
\text { Handelnder }\end{array}$ & 27,16 & 18,35 & 0 & 81,25 & 32,44 & 16,94 & 0 & 70,83 & 0.160903 \\
\hline
\end{tabular}

Tab. 9 Erreichte Lernziele des Abschnitt 1 nach Geschlecht; Mittelwerte \pm Standardabweichungen, Minima und Maxima dargestellt in \%. 
Ebenso zeigt sich in Abschnitt 2, dass Männer angeben im Durchschnitt mehr Lernziele erreicht zu haben als Frauen (Abb. 18).

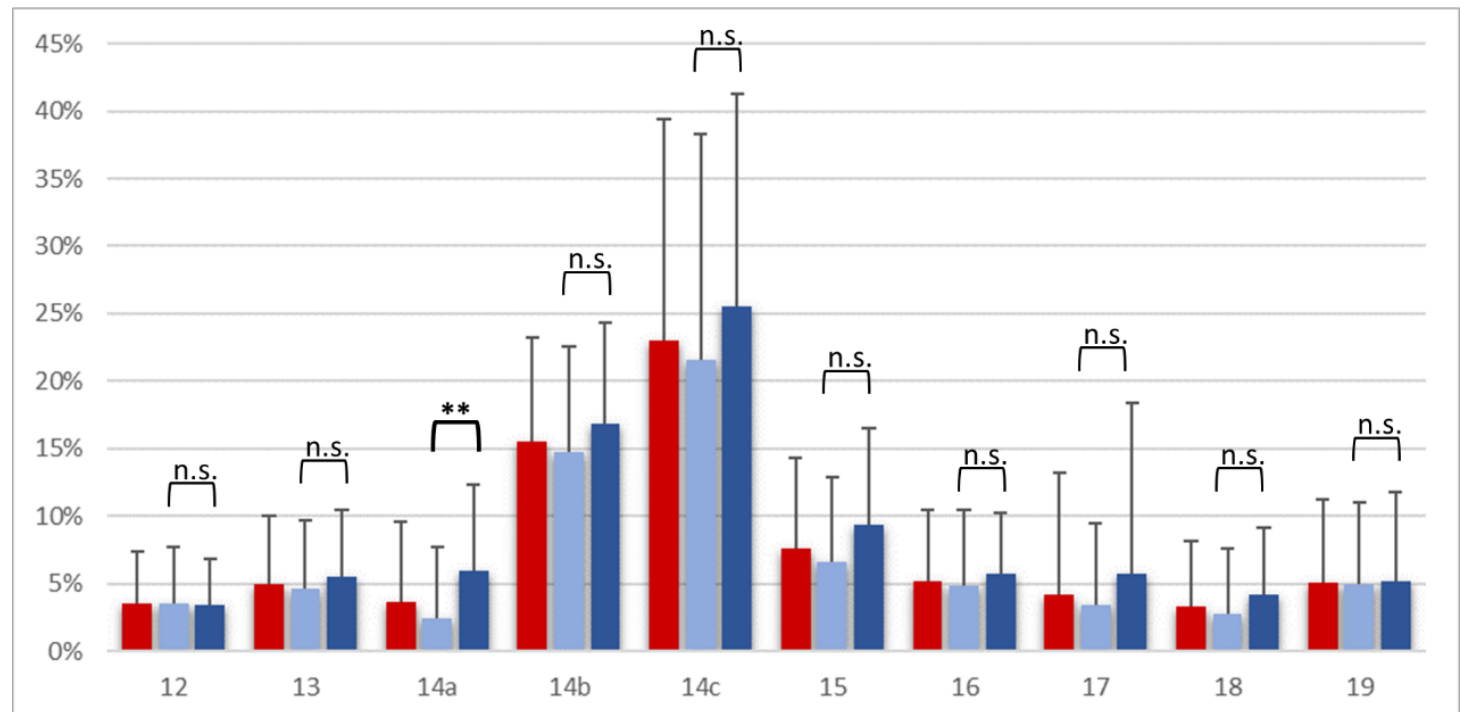

Abb. 18 Erreichte Lernziele in Abschnitt 2 nach Geschlecht; Mittelwerte + Standardabweichungen dargestellt in \%; rot $=$ gesamt; hellblau $=$ weiblich, dunkelblau $=$ männlich. ${ }^{* *} p<0.01,{ }^{*} p<0.05={ }^{*}$, n.s. $p>0.05$.

\begin{tabular}{|c|c|c|c|c|c|c|c|c|c|}
\hline \multirow[t]{2}{*}{ Kapitel } & \multicolumn{4}{|l|}{ Frauen } & \multicolumn{5}{|c|}{ Männer } \\
\hline & MW & SD & Min. & Max & MW & SD & Min. & Max. & $\mathbf{P}$ \\
\hline 12 Physiologie & 3,60 & 4,15 & 0 & 21,41 & 3,47 & 3,35 & 0 & 12,90 & 0.718662 \\
\hline $\begin{array}{l}13 \text { Patho- } \\
\text { physiologie }\end{array}$ & 4,61 & 5,14 & 0 & 23,49 & 5,53 & 4,97 & 0 & 21,48 & 0.229155 \\
\hline $\begin{array}{l}\text { 14a Medizinisch- } \\
\text { wissenschaftliche } \\
\text { Fertigkeiten }\end{array}$ & 2,46 & 5,27 & 0 & 24,32 & 5,98 & 6,37 & 0 & 21,62 & 0.005902 \\
\hline $\begin{array}{l}\text { 14b Klinisch- } \\
\text { praktische } \\
\text { Fertigkeiten }\end{array}$ & 14,79 & 7,83 & 0 & 41,30 & 16,82 & 7,52 & 3,30 & 39,13 & 0.111331 \\
\hline $\begin{array}{l}\text { 14c Ärztliche } \\
\text { Gesprächsführung }\end{array}$ & 21,60 & 16,69 & 0 & 70,69 & 25,58 & 15,69 & 0,86 & 68,97 & 0.206834 \\
\hline 15 Diagnostik & 6,66 & 6,23 & 0 & 31,87 & 9,38 & 7,13 & 0 & 26,37 & 0.088626 \\
\hline 16 Therapie & 4,91 & 5,57 & 0 & 30,80 & 5,72 & 4,50 & 0 & 18,25 & 0.166954 \\
\hline 17 Notfälle & 3,46 & 5,98 & 0 & 27,78 & 5,75 & 12,68 & 0 & 66,67 & 0.443884 \\
\hline $\begin{array}{l}18 \text { Ethik, Recht } \\
\text { und Geschichte der } \\
\text { Medizin }\end{array}$ & 2,80 & 4,81 & 0 & 27,14 & 4,23 & 4,91 & 0 & 15,71 & 0.149296 \\
\hline
\end{tabular}


19 Gesundheits-

förderung und

Prävention

$\begin{array}{lllllllll}4,94 & 6,04 & 0 & 27,38 & 5,23 & 6,53 & 0 & 25,00 & 0.956720\end{array}$

Tab. 10 Erreichte Lernziele des Abschnitt 2 nach Geschlecht; Mittelwerte \pm Standardabweichungen, Minima und Maxima der Mittelwerte dargestellt in \%.

Besonders hervorzuheben sind an dieser Stelle die Klinisch-praktisch relevanten Kapitel 14 a- c Medizinisch-wissenschaftliche Fertigkeiten (14a), Klinisch-praktische Fertigkeiten (14b) und Ärztliche Gesprächsführung (14c) (Abb. 19).

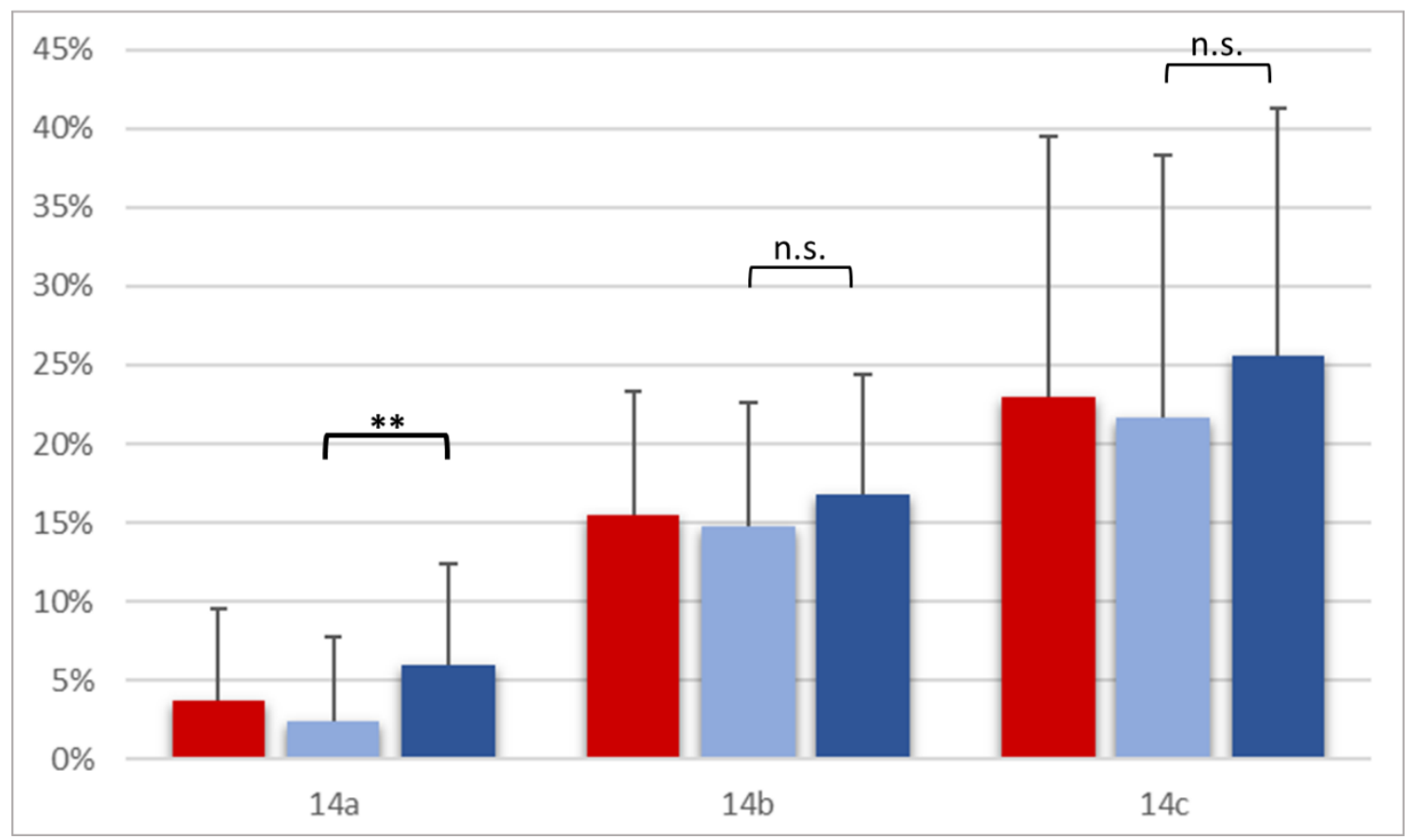

Abb. 19 Erreichte Lernziele des Abschnitt 2 (speziell Kapitel 14 a - c) nach Geschlecht; Mittelwerte + Standardabweichungen dargestellt in \%; rot = gesamt; hellblau = weiblich, dunkelblau = männlich . ${ }^{* *} p<0.01,{ }^{*} p<0.05={ }^{*}$, n.s. $p>0.05$.

Abschnitt 3 hingegen zeigt eine ausgeglichenere Abdeckung der erreichten Lernziele von Männern und Frauen. In Kapitel 20 der ärztlichen Konsultationsanlässe erreichen Männer 11,32+10,15\% (Min. = 0\%, Max. $=41,94 \%$ ) und Frauen 10,54+9,74\% (Min. = 0\%; Max. =51,61\%). In Kapitel 21 mit relevanten Krankheitsbildern erreichen Männer 5,08 $\pm 3,83 \%$ (Min. = 0,23\%; Max. $=16,33 \%$ ) und Frauen 4,17+3,20\% (Min. = 0,45\%; Max. = 16,78\%) (Abb. 20). 


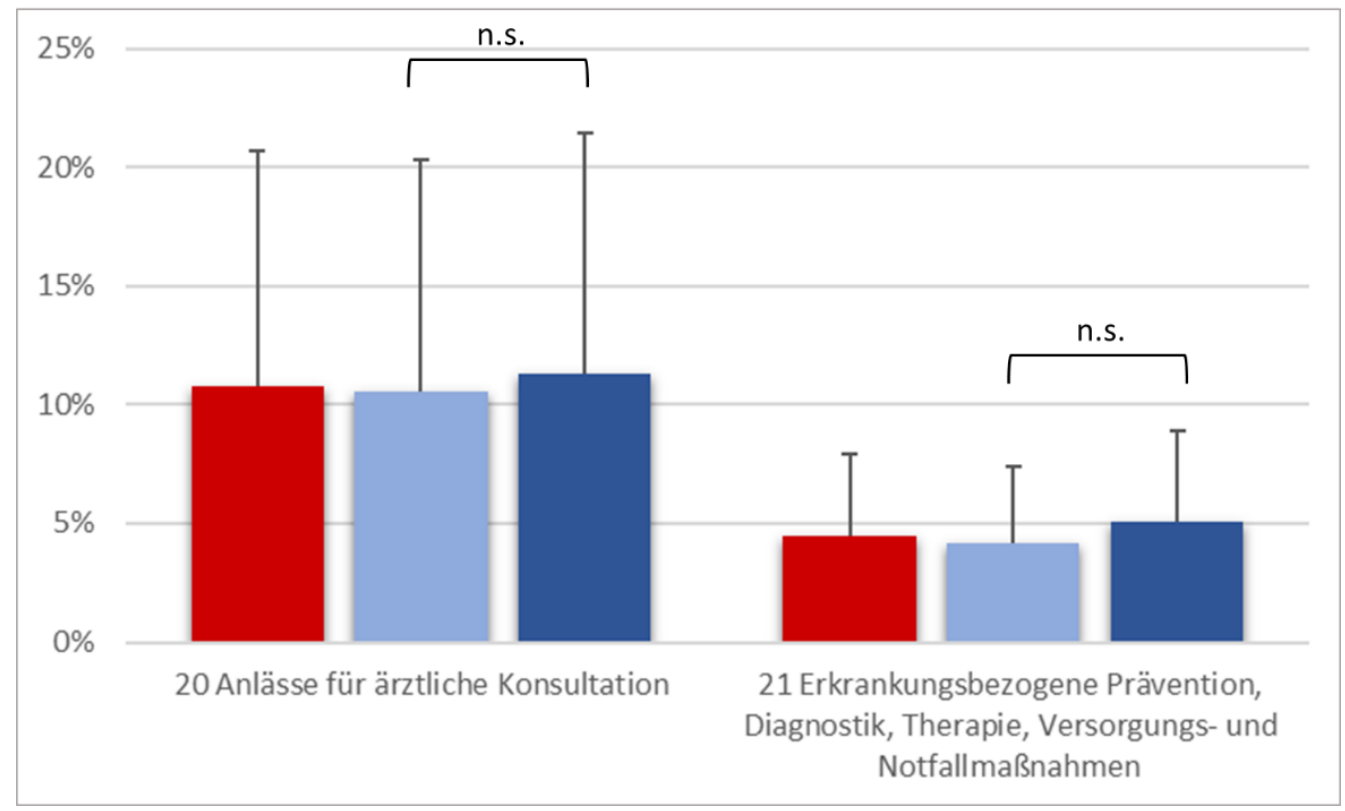

Abb. 20 Erreichte Lernziele des Abschnitt 3 nach Geschlecht; Mittelwerte + Standardabweichungen dargestellt in \%; rot = gesamt; hellblau = weiblich, dunkelblau = männlich; ${ }^{* *} p<0.01,{ }^{*} p<0.05=$ *, n.s. $p>0.05$

\subsubsection{Fachrichtungen gesamt}

In Bezug auf die unterschiedlichen chirurgischen Fachrichtungen, die im Rahmen des Blockpraktikums Chirurgie besucht werden können, konnten wir zeigen, dass Studierende, die durch mehrere Abteilungen/Stationen rotiert sind, mit 9,95+6,67\% (Min. = 1,90\%; Max. = 29,84\%) mehr Lernziele des NKLM abdecken konnten als Studierende, die nur in einem chirurgischen Fachgebiet ihr Blockpraktikum absolviert haben. Die Studienteilnehmer, die ihr BP Chirurgie in den Fachbereichen Allgemeinchirurgie, Unfallchirurgie und "Sonstige“ absolviert haben, konnten weniger Lernziele als im Durchschnitt vermittelt bekommen (Abb. 21) 


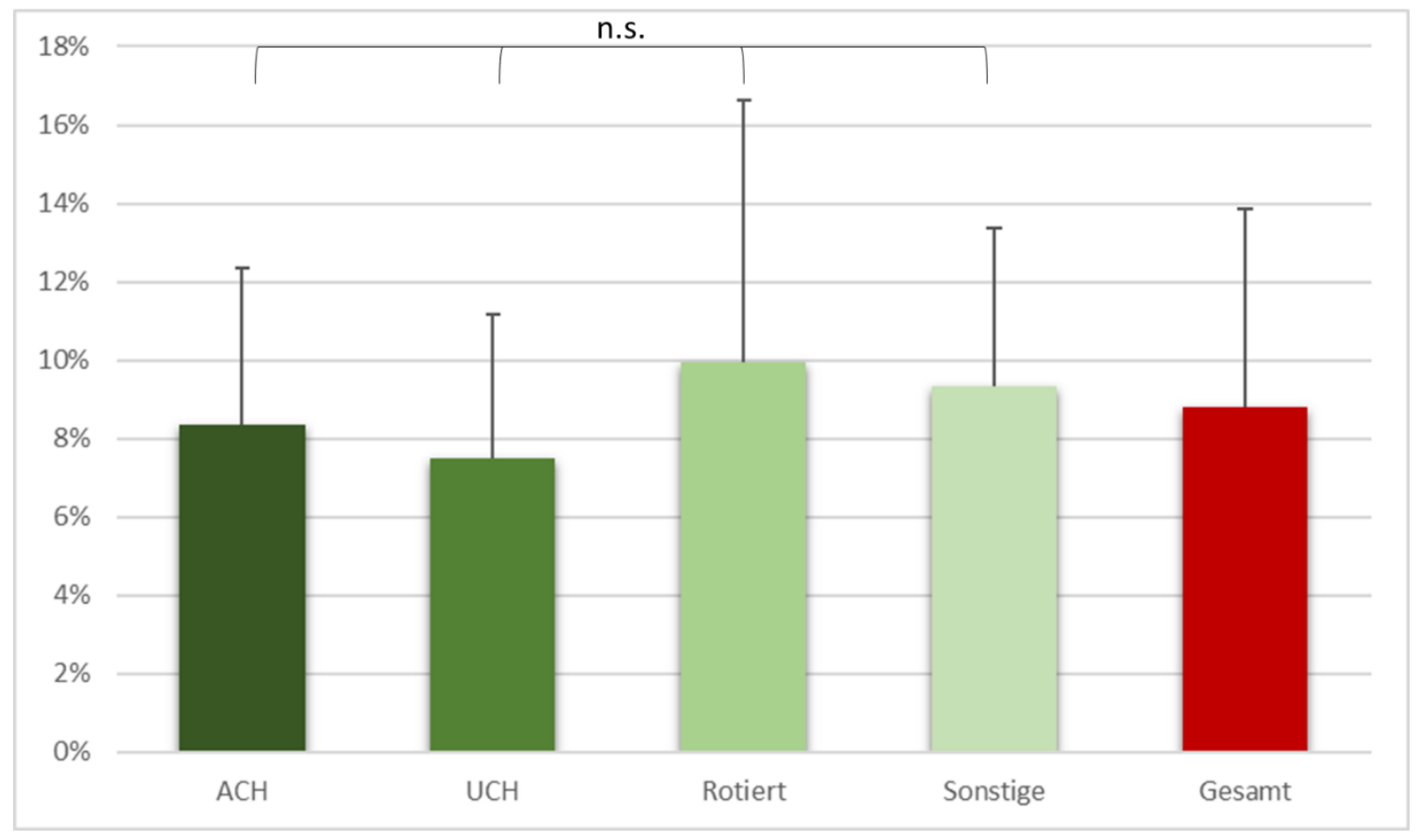

Abb. 21 Insgesamt erreichte Lernziele nach Fachabteilungen; Mittelwerte + Standardabweichungen dargestellt in \%; von links nach rechts $\mathrm{ACH}, \mathrm{UCH}$, Rotiert, Sonstige, Gesamt; ${ }^{* *} p<0.01,{ }^{*} p<0.05=$ *, n.s. $p>0.05$

\begin{tabular}{|llllll|}
\hline Fachrichtung & MW & $\mathbf{\pm S D}$ & Min. & Max. & $\mathbf{p}$ \\
\hline ACH & 8,35 & 4,01 & 2,95 & 17,66 & \\
UCH & 7,48 & 3,70 & 1,01 & 16,33 & \\
Rotiert & 9,95 & 6,67 & 1,90 & 29,84 & \\
Sonstige & 9,34 & 4,04 & 3,84 & 17,25 & \\
& & & & & 0.728997 \\
\hline
\end{tabular}

Tab. 11 Insgesamt erreichte Lernziele nach Fachrichtungen. Mittelwerte \pm Standardabweichungen, Minima und Maxima der Mittelwerte dargestellt in \%.

Für den ersten Abschnitt der ärztlichen Rollen des NKLM können unsere Ergebnisse zeigen, Studierende aus "Sonstigen“ chirurgischen Fachrichtungen im Durchschnitt 35,60+14,27\% (Min. = 11,17\%; Max.=60,11\%) am meisten Lernziele abdecken konnten (Abb. 22) 


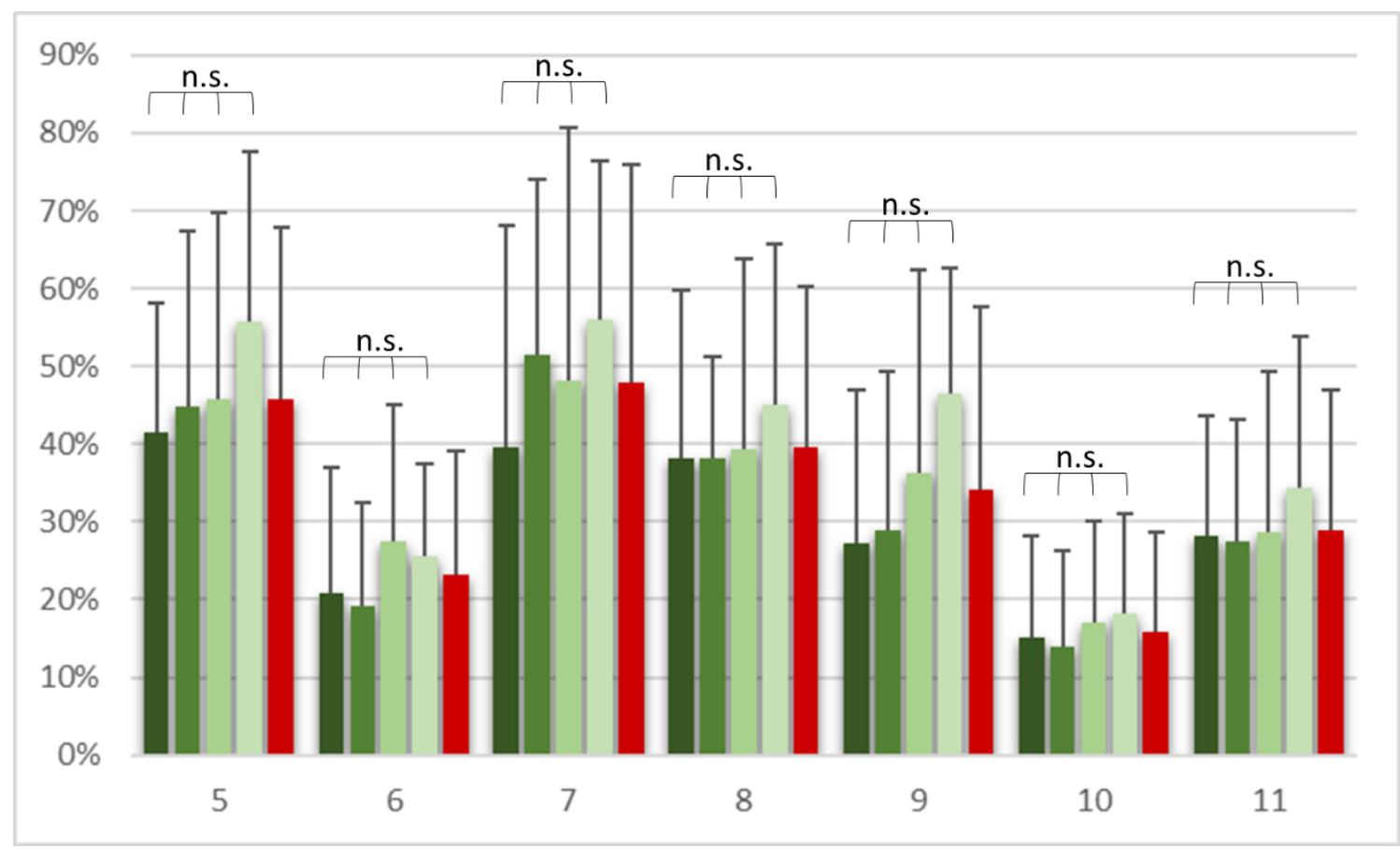

Abb. 22 Erreichte Lernziele des Abschnitt 1 nach Fachabteilungen. Mittelwerte + Standardabweichungen dargestellt in \%; von links nach rechts $\mathrm{ACH}, \mathrm{UCH}$, Rotiert, Sonstige und Gesamt; ${ }^{* *} p<0.01,{ }^{*} p<0.05={ }^{*}$, n.s. $p>0.05$

\begin{tabular}{|c|c|c|c|c|c|}
\hline 5 Medizinischer Experte & MW & SD & $\min$ & $\max$ & $\mathbf{p}$ \\
\hline $\mathrm{ACH}$ & 41,47 & 16,54 & 4,17 & 66,67 & \\
\hline UCH & 44,89 & 22,58 & 8,33 & 91,67 & \\
\hline Rotiert & 45,83 & 23,92 & 0,00 & 79,17 & \\
\hline \multirow[t]{2}{*}{ Sonstige } & 55,68 & 22,00 & 12,50 & 79,17 & \\
\hline & & & & & 0.296066 \\
\hline 6 Gelehrter & MW & SD & $\min$ & Max & $\mathbf{p}$ \\
\hline $\mathrm{ACH}$ & 20,89 & 16,10 & 0,00 & 48,39 & \\
\hline UCH & 19,21 & 13,21 & 0,00 & 48,39 & \\
\hline Rotiert & 27,48 & 17,65 & 0,00 & 67,74 & \\
\hline \multirow[t]{2}{*}{ Sonstige } & 25,51 & 11,87 & 0,00 & 51,61 & \\
\hline & & & & & 0.250582 \\
\hline 7 Kommunikator & MW & SD & $\min$ & $\max$ & $\mathbf{p}$ \\
\hline $\mathrm{ACH}$ & 39,68 & 28,39 & 0,00 & 100,00 & \\
\hline UCH & 51,52 & 22,42 & 16,67 & 100,00 & \\
\hline Rotiert & 48,15 & 32,50 & 0,00 & 100,00 & \\
\hline \multirow[t]{2}{*}{ Sonstige } & 56,06 & 20,44 & 16,67 & 83,33 & \\
\hline & & & & & 0.340457 \\
\hline 8 Mitglied eines Teams & MW & SD & $\min$ & Max & $\mathbf{p}$ \\
\hline $\mathrm{ACH}$ & 38,25 & 21,60 & 4,17 & 78,26 & \\
\hline
\end{tabular}




\begin{tabular}{|c|c|c|c|c|c|}
\hline $\mathrm{UCH}$ & 38,25 & 13,01 & 16,67 & 73,91 & \\
\hline Rotiert & 39,29 & 24,59 & 0,00 & 78,26 & \\
\hline \multirow[t]{2}{*}{ Sonstige } & 45,03 & 20,69 & 12,50 & 78,26 & \\
\hline & & & & & 0.807009 \\
\hline $\begin{array}{l}9 \text { Gesundheitsberater und } \\
\text { /-fürsorger }\end{array}$ & MW & SD & $\min$ & $\max$ & $\mathbf{P}$ \\
\hline $\mathrm{ACH}$ & 27,25 & 19,77 & 0,00 & 72,22 & \\
\hline $\mathrm{UCH}$ & 28,79 & 20,63 & 5,56 & 88,89 & \\
\hline Rotiert & 36,21 & 26,25 & 0,00 & 88,89 & \\
\hline \multirow[t]{2}{*}{ Sonstige } & 46,46 & 16,29 & 22,22 & 77,78 & \\
\hline & & & & & 0.080719 \\
\hline $\begin{array}{l}10 \text { Verantwortungsträger } \\
\text { und Manager }\end{array}$ & MW & SD & $\min$ & $\max$ & $\mathbf{P}$ \\
\hline $\mathrm{ACH}$ & 15,19 & 13,01 & 0,00 & 48,65 & \\
\hline UCH & 14,00 & 12,21 & 0,00 & 54,05 & \\
\hline Rotiert & 16,92 & 13,13 & 0,00 & 43,24 & \\
\hline \multirow[t]{2}{*}{ Sonstige } & 18,18 & 12,89 & 0,00 & 40,54 & \\
\hline & & & & & 0.785139 \\
\hline \multicolumn{6}{|l|}{11 Professionell } \\
\hline $\mathrm{ACH}$ & 28,17 & 15,34 & 0,00 & 62,50 & \\
\hline $\mathrm{UCH}$ & 27,56 & 15,72 & 0,00 & 62,50 & \\
\hline Rotiert & 28,63 & 20,61 & 0,00 & 81,25 & \\
\hline \multirow[t]{2}{*}{ Sonstige } & 34,28 & 19,47 & 14,58 & 70,83 & \\
\hline & & & & & 0.885895 \\
\hline
\end{tabular}

Tab. 12 Erreichte Lernziele des Abschnitt 1 nach Fachrichtungen; Mittelwerte, \pm Standardabweichungen, Minima und Maxima dargestellt in \%.

Die Auswertungen in Abschnitt 2 zeigen, dass Studierende bei Rotation im BP Chirurgie mit 7,98+6,69\% (Min $=0,00 \%$; Max. $=30,10 \%)$ mehr Lernziele als "Gesamt" abdecken können. Ebenfalls lässt sich darstellen, dass vor allem im Fachbereich Unfallchirurgie mit 5,61 $\pm 3,67 \%$ (Min. $=0,30 \%$; Max. $=15,49 \%$ ) und auch im Bereich Allgemeinchirurgie mit 6,47 $\pm 3,51 \%$ (Min. = 2,38\%; Max. = 17,49\%) deutlich weniger Lernziele als im Gesamtdurchschnitt vermittelt werden können (Abb. 23) 


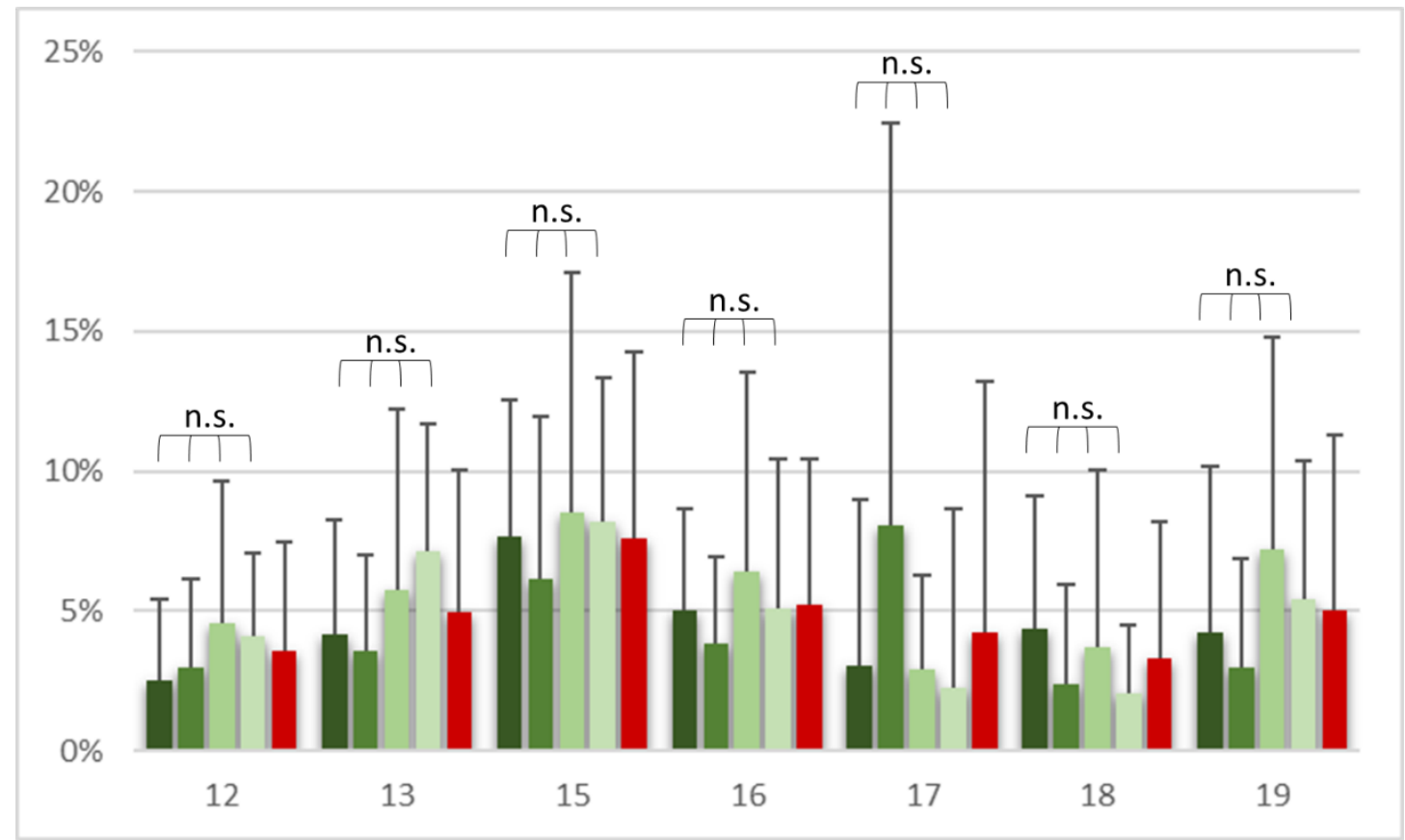

Abb. 23 Erreichte Lernziele des Abschnitt 2 (Kap. 12, 13, 15 - 19) nach Fachabteilungen; Mittelwerte + Standardabweichungen dargestellt in \%; von links nach rechts $\mathrm{ACH}, \mathrm{UCH}$, Rotiert, Sonstige und Gesamt. ${ }^{* *} p<0.01,{ }^{*} p<0.05$, n.s. $p>0.05$.

Die klinisch-praktisch relevanten Kapitel $14 \mathrm{a}-\mathrm{c}$, Medizinisch-wissenschaftliche Fertigkeiten (14a), Klinisch-praktische Fertigkeiten (14b) und Ärztliche Gesprächsführung (14c), zeigen das besonders deutlich (Abb. 24) 


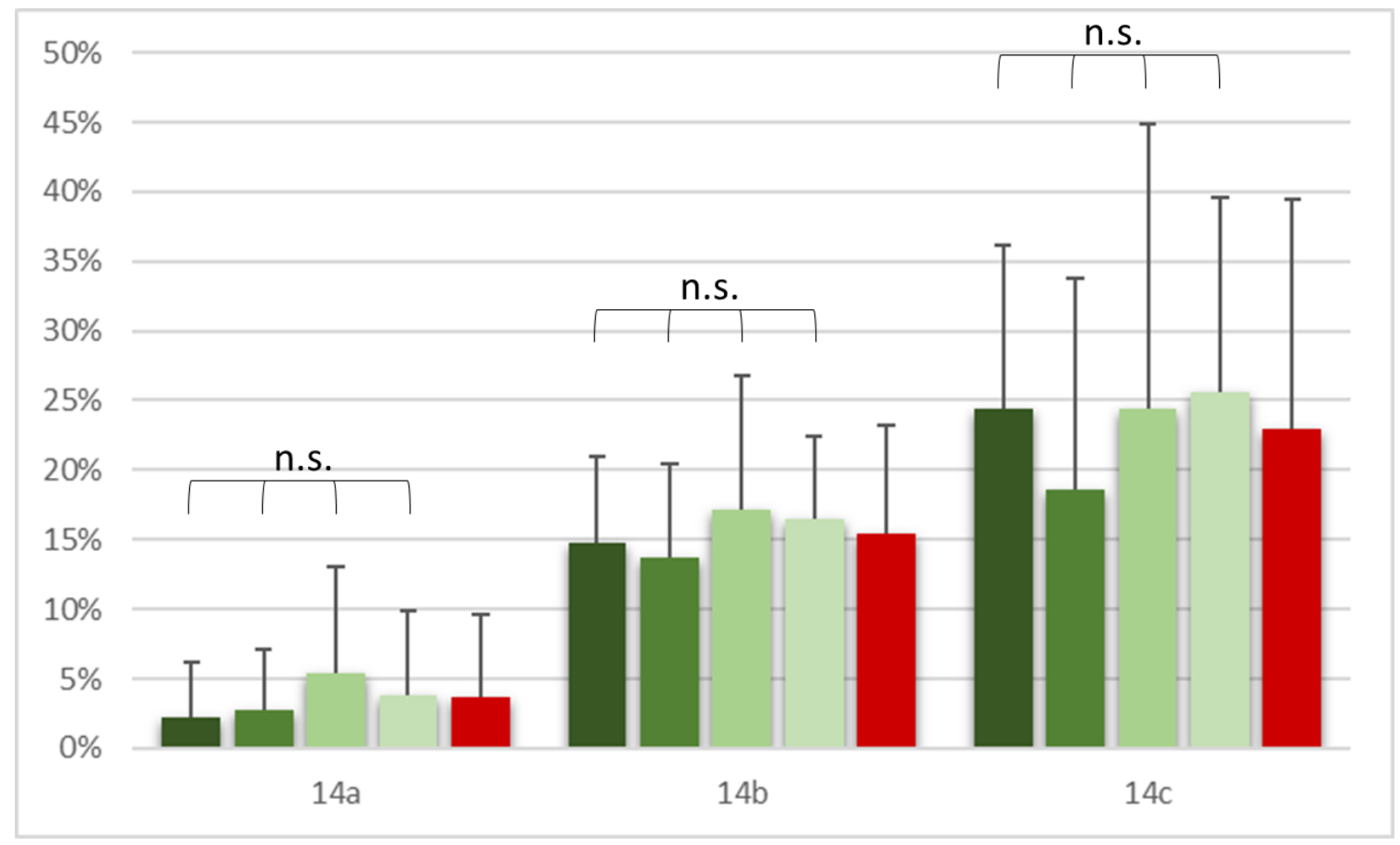

Abb. 24 Erreichte Lernziele der Kapitel 14 a - c nach Fachabteilungen. Mittelwerte + Standardabweichungen dargestellt in \%; von links nach rechts $\mathrm{ACH}, \mathrm{UCH}$, Rotiert, Sonstige und Gesamt. ** $p<0.01,{ }^{*} p<0.05$, n.s. $p>0.05$.

\begin{tabular}{|lccccc|}
\hline 12 Physiologie & MW & SD & min & max & P \\
\hline ACH & 2,54 & 2,85 & 0,00 & 13,14 & \\
UCH & 2,99 & 3,14 & 0,00 & 12,90 & \\
Rotiert & 4,58 & 5,03 & 0,00 & 21,41 & \\
Sonstige & 4,12 & 2,93 & 0,00 & 9,98 & \\
& & & & & 0.536292 \\
\hline 13 Pathophysiologie & MW & SD & min & Max & P \\
\hline ACH & 4,18 & 4,06 & 0,67 & 14,77 & \\
UCH & 3,56 & 3,45 & 0,00 & 14,09 & \\
Rotiert & 5,71 & 6,51 & 0,00 & 23,49 & \\
Sonstige & 7,14 & 4,56 & 0,67 & 14,09 & \\
& & & & & 0.269269 \\
\hline 14a Medizinisch-wissenschaftliche & & & & & \\
Fertigkeiten & MW & SD & min & max & P \\
\hline ACH & 2,29 & 3,88 & 0,00 & 13,16 & \\
UCH & 2,79 & 4,34 & 0,00 & 13,16 & \\
\hline Rotiert & 5,42 & 7,59 & 0,00 & 24,32 & \\
\hline Sonstige & 3,85 & 6,09 & 0,00 & 21,05 & \\
\hline & & & & & 0.399086 \\
\hline 14b Klinisch-praktische Fertigkeiten & MW & SD & min & Max & P \\
\hline ACH & 14,73 & 6,27 & 6,59 & 30,43 & \\
\hline & & & & & \\
\hline
\end{tabular}




\begin{tabular}{|c|c|c|c|c|c|}
\hline UCH & 13,74 & 6,69 & 3,30 & 30,43 & \\
\hline Rotiert & 17,09 & 9,73 & 0,00 & 41,30 & \\
\hline \multirow[t]{2}{*}{ Sonstige } & 16,53 & 5,92 & 8,70 & 31,52 & \multirow[b]{2}{*}{0.473676} \\
\hline & & & & & \\
\hline 14c Ärztliche Gesprächsführung & MW & SD & $\min$ & $\max$ & $\mathbf{P}$ \\
\hline $\mathrm{ACH}$ & 24,38 & 11,78 & 5,17 & 44,83 & \\
\hline $\mathrm{UCH}$ & 18,61 & 15,16 & 0,86 & 58,62 & \\
\hline Rotiert & 24,39 & 20,41 & 0,00 & 70,69 & \\
\hline \multirow[t]{2}{*}{ Sonstige } & 25,55 & 13,98 & 5,17 & 54,31 & \\
\hline & & & & & 0.346417 \\
\hline 15 Diagnostik & MW & SD & $\min$ & Max & $\mathbf{p}$ \\
\hline $\mathrm{ACH}$ & 7,64 & 4,92 & 0,00 & 19,78 & \\
\hline UCH & 6,14 & 5,79 & 0,00 & 23,08 & \\
\hline Rotiert & 8,51 & 8,62 & 1,10 & 31,87 & \\
\hline \multirow[t]{2}{*}{ Sonstige } & 8,19 & 5,14 & 0,00 & 18,68 & \\
\hline & & & & & 0.585295 \\
\hline 16 Therapie & MW & SD & $\min$ & $\max$ & $\mathbf{p}$ \\
\hline $\mathrm{ACH}$ & 5,05 & 3,61 & 0,76 & 11,79 & \\
\hline UCH & 3,84 & 3,10 & 0,00 & 11,03 & \\
\hline Rotiert & 6,44 & 7,07 & 0,00 & 30,80 & \\
\hline \multirow[t]{2}{*}{ Sonstige } & 5,12 & 5,28 & 0,38 & 20,15 & \\
\hline & & & & & 0.725811 \\
\hline 17 Notfälle & MW & SD & $\min$ & $\max$ & $\mathbf{p}$ \\
\hline $\mathrm{ACH}$ & 3,04 & 5,93 & 0,00 & 27,78 & \\
\hline $\mathrm{UCH}$ & 8,08 & 14,38 & 0,00 & 66,67 & \\
\hline Rotiert & 2,88 & 3,42 & 0,00 & 13,89 & \\
\hline \multirow[t]{2}{*}{ Sonstige } & 2,27 & 6,36 & 0,00 & 22,22 & \\
\hline & & & & & 0.070509 \\
\hline 18 Recht, Ethik und Geschichte & MW & SD & $\min$ & Max & $\mathbf{p}$ \\
\hline $\mathrm{ACH}$ & 4,35 & 4,74 & 0,00 & 17,14 & \\
\hline UCH & 2,40 & 3,51 & 0,00 & 15,71 & \\
\hline Rotiert & 3,70 & 6,31 & 0,00 & 27,14 & \\
\hline \multirow[t]{2}{*}{ Sonstige } & 2,08 & 2,39 & 0,00 & 7,14 & \\
\hline & & & & & 0.339474 \\
\hline $\begin{array}{l}19 \text { Gesundheitsförderung und } \\
\text { Prävention }\end{array}$ & MW & SD & $\min$ & $\max$ & $\mathbf{p}$ \\
\hline $\mathrm{ACH}$ & 4,25 & 5,93 & 0,00 & 22,62 & \\
\hline $\mathrm{UCH}$ & 2,98 & 3,86 & 0,00 & 13,10 & \\
\hline Rotiert & 7,19 & 7,60 & 0,00 & 27,38 & \\
\hline \multirow[t]{2}{*}{ Sonstige } & 5,41 & 4,96 & 0,00 & 13,10 & \\
\hline & & & & & 0.172740 \\
\hline
\end{tabular}

Tab. 13 Erreichte Lernziele des Abschnitt 2 nach Fachrichtungen. Mittelwerte \pm

Standardabweichungen, Minima und Maxima dargestellt in \%. 
Für die Lernziele des Abschnitt 3 lässt sich sagen, dass Studierende in „Sonstige“ eher kleineren und spezialisierteren Fachrichtungen mit 6,16+4,49\% (Min. = 0,00\%; Max. $=12,90 \%$ ) am wenigsten Anlässe ärztlicher Konsultation (Kapitel 20) und mit 2,68 $\pm 1,68 \%$ (Min. $=0,68 \%$; Max. $=7,03 \%$ ) relevante Krankheitsbilder (Kapitel 21) in ihrem BP Chirurgie bearbeiten konnten. Die Abdeckung in den Fachrichtungen $\mathrm{ACH}$, $\mathrm{UCH}$ und „Rotiert“ zeigen sich relativ homogen verteilt und entsprechen ungefähr dem erreichten Durchschnitt (Abb. 25).

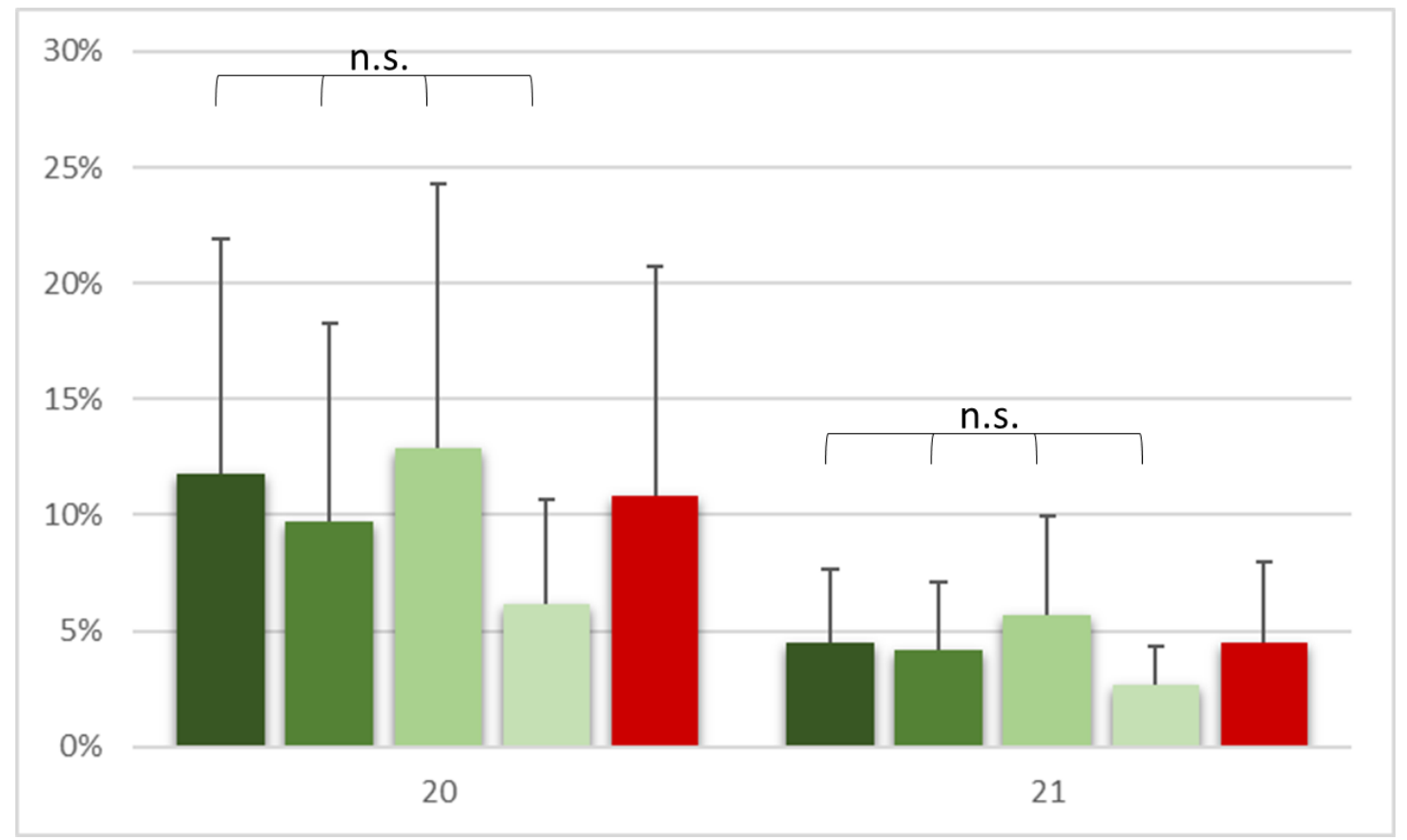

Abb. 25 Erreichte Lernziele des Abschnitt 3 nach Fachabteilungen. Mittelwerte + Standardabweichungen dargestellt in \%; von links nach rechts $\mathrm{ACH}, \mathrm{UCH}$, Rotiert, Sonstige und Gesamt. ${ }^{* *} p<0.01,{ }^{*} p<0.05$, n.s. $p>0.05$.

\begin{tabular}{|lccccc|}
\hline $\mathbf{2 0}$ Ärztliche Konsultationsanlässe & MW & SD & $\min$ & $\max$ & $\mathbf{p}$ \\
\hline ACH & 11,75 & 10,17 & 0,00 & 41,94 & \\
UCH & 9,68 & 8,62 & 0,00 & 29,84 & \\
Rotiert & 12,90 & 11,38 & 0,00 & 51,61 & \\
Sonstige & 6,16 & 4,49 & 0,00 & 12,90 & \\
& & & & & 0.260346 \\
\hline
\end{tabular}




\begin{tabular}{|c|c|c|c|c|c|}
\hline $\begin{array}{l}21 \text { Erkrankungsbezo } \\
\text { Prävention, Diagnos } \\
\text { Versorgungs- und } \\
\text { Notfallmaßnahmen }\end{array}$ & MW & SD & $\min$ & Max & $p$ \\
\hline $\mathrm{ACH}$ & 4,52 & 3,12 & 0,45 & 12,24 & \\
\hline $\mathrm{UCH}$ & 4,16 & 2,94 & 0,23 & 13,61 & \\
\hline Rotiert & 5,65 & 4,30 & 0,45 & 16,78 & \\
\hline \multirow[t]{2}{*}{ Sonstige } & 2,68 & 1,68 & 0,68 & 7,03 & \\
\hline & & & & & 0.127824 \\
\hline
\end{tabular}

Tab. 14 Erreichte Lernziele des Abschnitt 3 nach Fachrichtungen. Mittelwerte \pm Standardabweichungen, Minima und Maxima dargestellt in \%.

Die Lernziele der Unterkapitel des Kapitel 21 nach Primären Organsystemen zeigen dem Spektrum der Fachrichtung entsprechend unterschiedliche Auslastungen. Die fachrichtungs-spezifische Auslastung der Lernziele zeigt sich vor allem in den Unterkapiteln „Kardiovaskulär“, „Muskuloskelettal und Weichgewebe“ und „Verdauungssysteme“ (Abb. 26).

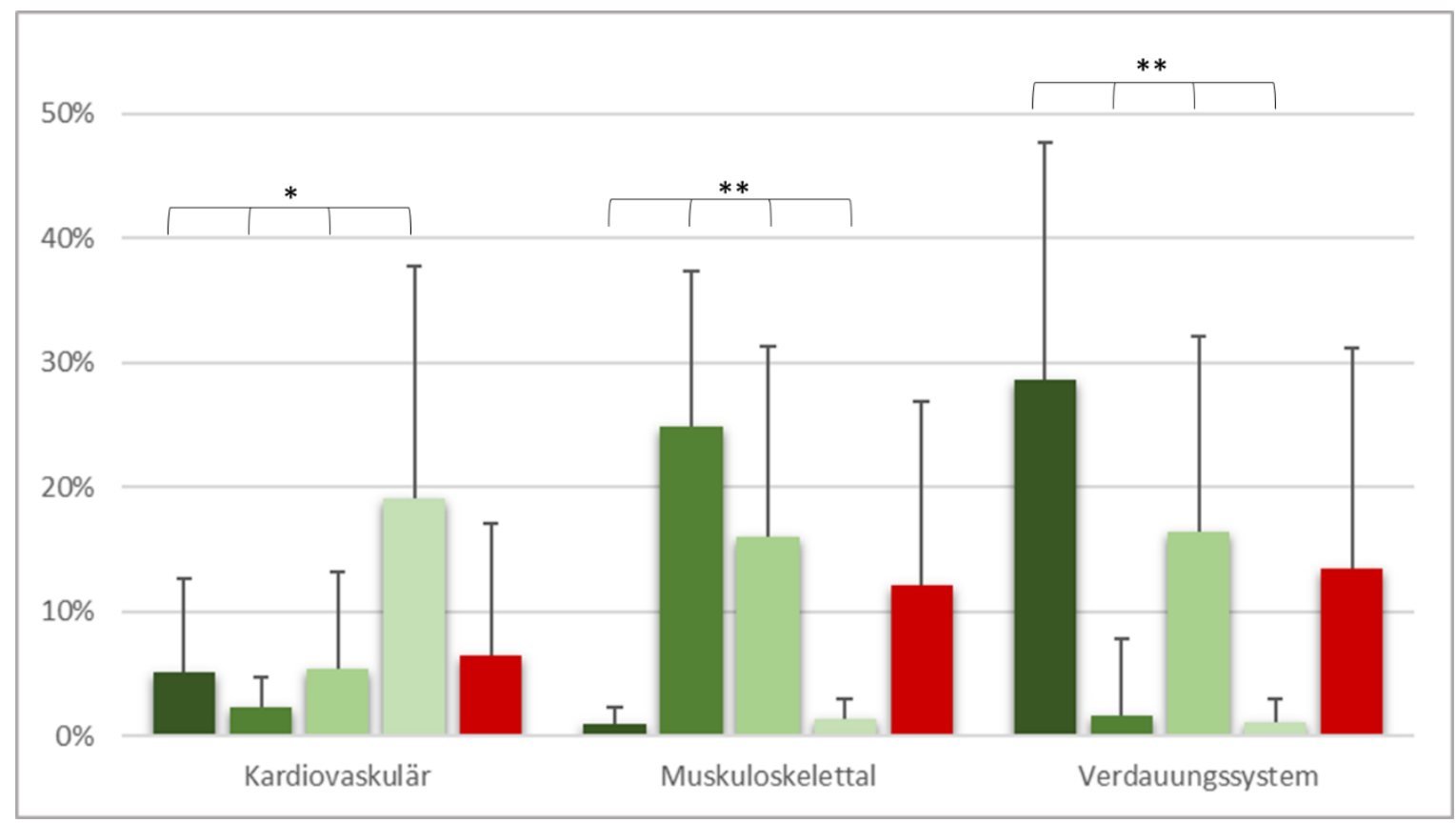

Abb. 26 Erreichte Lernziele ausgewählter Unterkapiteln des Kapitel 21 zu Primären Organsystemen nach Fachabteilungen. Mittelwerte + Standardabweichungen dargestellt in \%; von links nach rechts $\mathrm{ACH}, \mathrm{UCH}$, Rotiert, Sonstige und Gesamt. ${ }^{* *} p<0.01,{ }^{*} p<0.05$, n.s. $p>0.05$. 


\begin{tabular}{|c|c|c|c|c|c|}
\hline $\begin{array}{l}21 \text { - Kardiovaskuläres } \\
\text { System }\end{array}$ & MW & SD & $\min$ & $\max$ & $\mathbf{p}$ \\
\hline Gesamt & 6,44 & 10,69 & 0,00 & 65,52 & \\
\hline $\mathrm{ACH}$ & 5,09 & 7,50 & 0,00 & 24,14 & \\
\hline $\mathrm{UCH}$ & 2,30 & 2,46 & 0,00 & 6,90 & \\
\hline Rotiert & 5,40 & 7,73 & 0,00 & 34,48 & \\
\hline \multirow[t]{2}{*}{ Sonstige } & 19,12 & 18,62 & 0,00 & 65,52 & \\
\hline & & & & & 0.011811 \\
\hline $\begin{array}{l}21 \text { Muskuloskelettal } \\
\text { und Weichgewebe }\end{array}$ & MW & SD & $\min$ & Max & $\mathbf{p}$ \\
\hline Gesamt & 12,17 & 14,66 & 0,00 & 62,50 & \\
\hline $\mathrm{ACH}$ & 0,99 & 1,38 & 0,00 & 4,17 & \\
\hline $\mathrm{UCH}$ & 24,80 & 12,53 & 2,08 & 50,00 & \\
\hline Rotiert & 16,03 & 15,25 & 0,00 & 62,50 & \\
\hline \multirow[t]{2}{*}{ Sonstige } & 1,33 & 1,61 & 0,00 & 4,17 & \\
\hline & & & & & $\mathrm{p}<0.001$ \\
\hline \multicolumn{6}{|l|}{21} \\
\hline Verdauungssystem & MW & SD & $\min$ & $\max$ & p \\
\hline Gesamt & 13,51 & 17,70 & 0,00 & 81,25 & \\
\hline $\mathrm{ACH}$ & 28,67 & 19,04 & 0,00 & 81,25 & \\
\hline UCH & 1,69 & 6,19 & 0,00 & 29,17 & \\
\hline Rotiert & 16,39 & 15,76 & 0,00 & 70,83 & \\
\hline \multirow[t]{2}{*}{ Sonstige } & 1,14 & 1,86 & 0,00 & 4,17 & \\
\hline & & & & & $p<0.001$ \\
\hline
\end{tabular}

Tab. 15 Erreichte Lernziele ausgewählter Unterkapitel des Kapitel 21 nach Fachrichtungen.

Mittelwerte \pm Standardabweichungen, Minima und Maxima dargestellt in \%.

\subsubsection{Uni/Lehrkrankenhäuser gesamt}

Im Mittel geben die Studierenden an, an der Universitätsklinik Frankfurt am Main, an Lehrkrankenhäusern mit über 200 chirurgischen Betten und an Lehrkrankenhäusern mit 100 - 200 chirurgischen Betten ca. 7 - 9\% und damit weniger als der im Durchschnitt erreichten Lernziele vermittelt bekommen $\mathrm{zu}$ haben. In Lehrkrankenhäusern mit weniger als 100 chirurgischen Bettenplätzen konnten Studienteilnehmer angeben mit 10,6 $\pm 6,75 \%$ (Min = 2,33\%; Max. $=29,84 \%)$, mehr Lernziele als im Gesamtdurchschnitt erreicht zu haben (Abb. 27). 


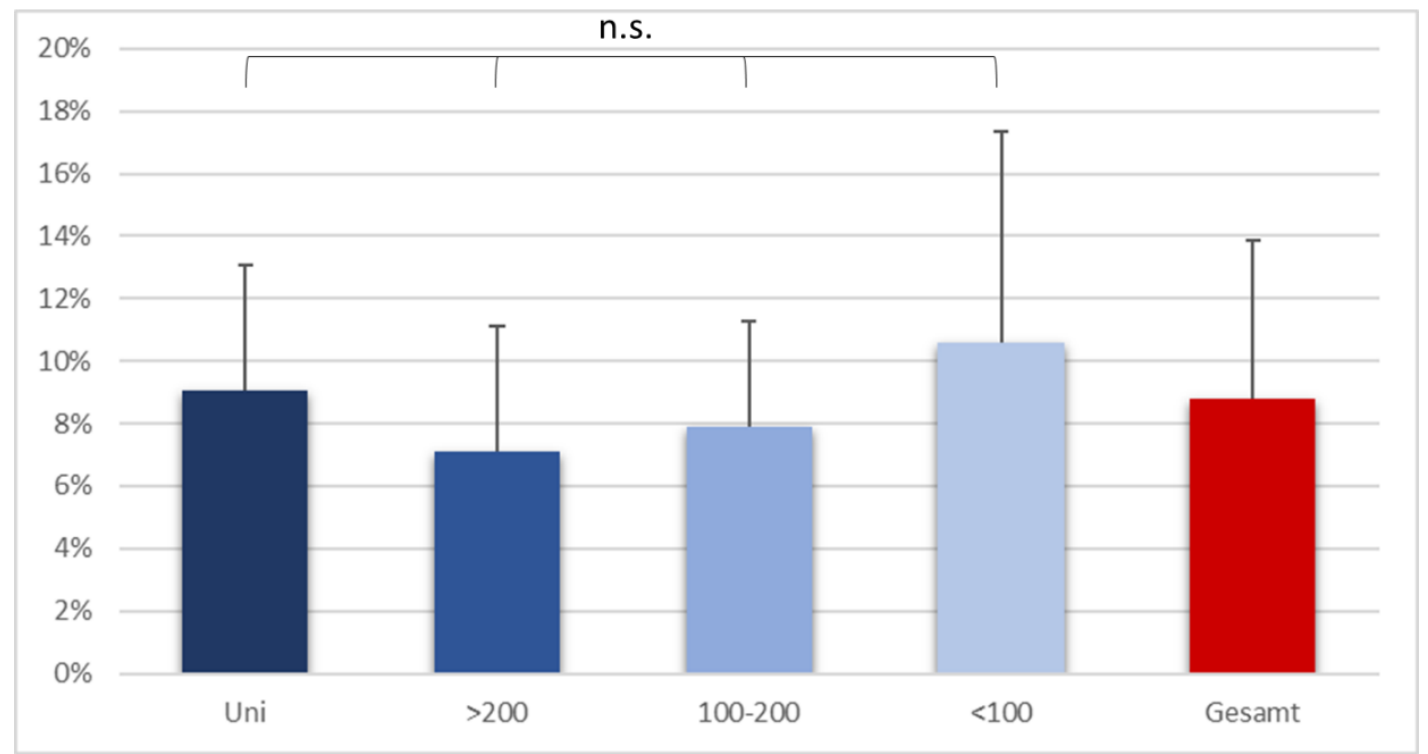

Abb. 27 Insgesamt erreichte Lernziele nach (Lehr-) Krankenhäusern. Mittelwerte + Standardabweichungen dargestellt in \%; von links nach rechts Uniklinik, >200, 100-200, <100 und Gesamt. ${ }^{* *} p<0.01,{ }^{*} p<0.05$, n.s. $p>0.05$.

\begin{tabular}{|lccccc|}
\hline (Lehr-)Krankenhaus & MW & SD & Min. & Max. & p \\
\hline Uniklinik & 9,04 & 4,04 & 3,84 & 17,25 & \\
$>\mathbf{2 0 0}$ & 7,13 & 4,00 & 1,01 & 14,94 & \\
$\mathbf{1 0 0 - 2 0 0}$ & 7,92 & 3,34 & 2,95 & 14,70 & \\
$<\mathbf{1 0 0}$ & 10,60 & 6,75 & 2,33 & 29,84 & \\
& & & & & 0.240458 \\
\hline
\end{tabular}

Tab. 16 Insgesamt erreichte Lernziele nach (Lehr-)Krankenhäusern. Mittelwerte,

Standardabweichungen, Minima und Maxima dargestellt in \%.

Bei Analyse der Kapitel des Abschnitts 1 zeigt sich im Vergleich der Lehrkrankenhaus Kategorien die folgende Verteilung. An der Uniklinik (MW 33,73 $\pm 15,27 \%$; Min = 11,17\%; Max. $=63,10 \%$ ) und an Lehrkrankenhäusern mit unter 100 chirurgischen Betten (MW 33,11 15,88\%; Min. = 3,72\%; Max. = 59,89\%) ist die Abdeckung der Lernziele im Mittel ähnlich hoch und höher als in Lehrkrankenhäusern mit mehr chirurgischen Bettenplätzen. Die im Mittel geringste Lernzielabdeckung weisen die Blockpraktika auf, die in Lehrkrankenhäusern mit

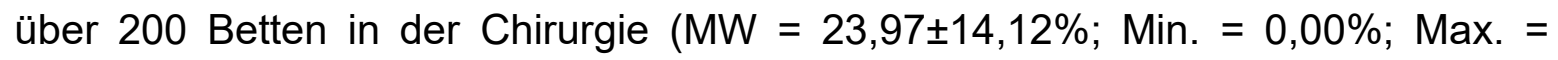
$50,80 \%$ ) absolviert wurden (Abb. 28). 


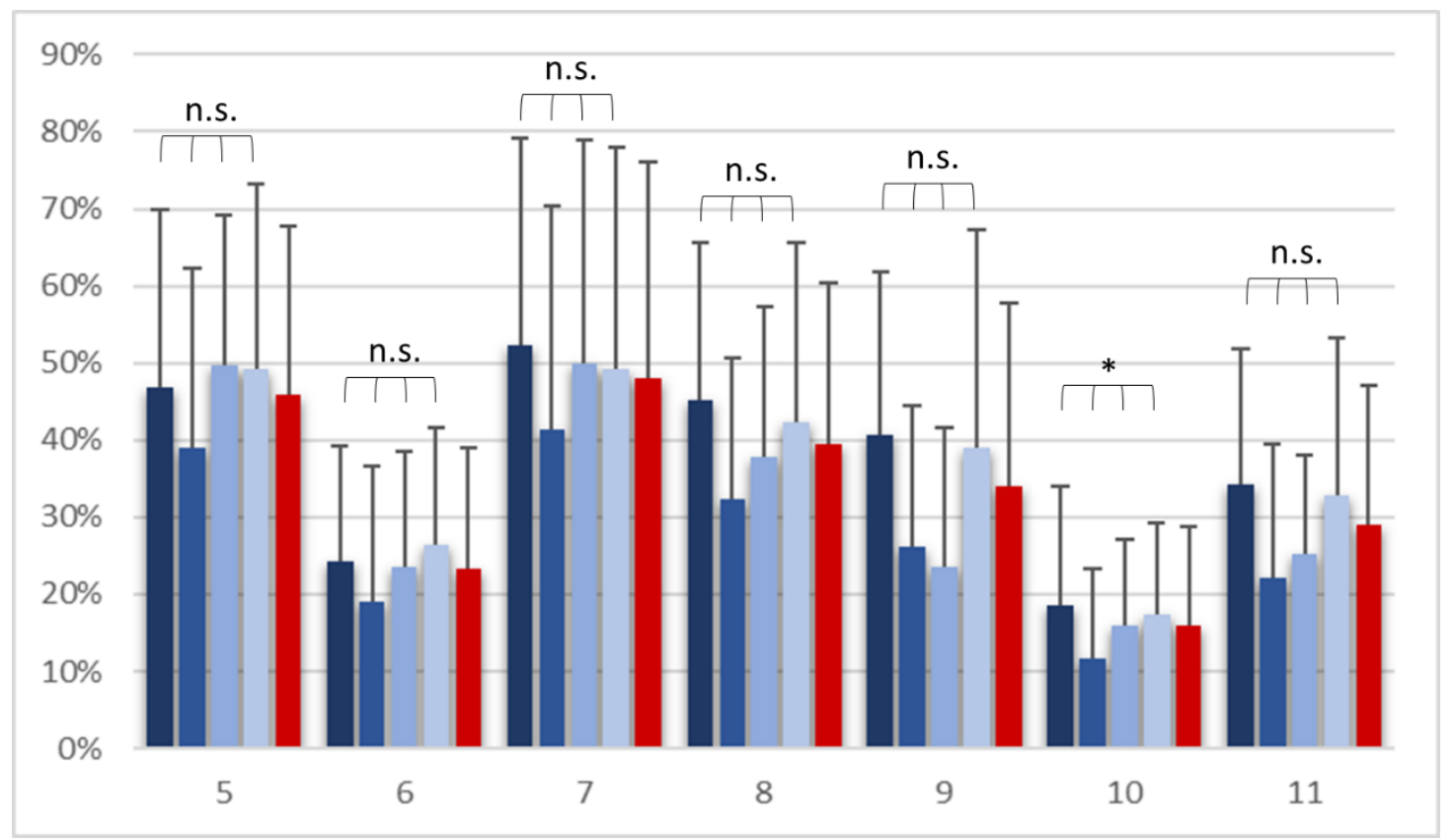

Abb. 28 Erreichte Lernziele des Abschnitt 1 nach (Lehr-)Krankenhäusern. Mittelwerte + Standardabweichungen dargestellt in \%; von links nach rechts Uniklinik, >200, 100-200, <100 und Gesamt. ${ }^{* *} p<0.01,{ }^{*} p<0.05$, n.s. $p>0.05$.

\begin{tabular}{|c|c|c|c|c|c|}
\hline 5 Medizinischer Experte & MW & SD & Min. & Max. & $\mathbf{p}$ \\
\hline Uniklinik & 46,83 & 23,00 & 8,33 & 91,67 & \\
\hline$>200$ & 38,95 & 23,45 & 0,00 & 79,17 & \\
\hline $100-200$ & 49,68 & 19,57 & 16,67 & 70,83 & \\
\hline \multirow[t]{2}{*}{$<100$} & 49,31 & 23,86 & 4,17 & 79,17 & \\
\hline & & & & & 0.349813 \\
\hline 6 Gelehrter & MW & SD & Min. & Max. & $\mathbf{p}$ \\
\hline Uniklinik & 24,27 & 14,92 & 0,00 & 51,61 & \\
\hline$>200$ & 18,93 & 17,61 & 0,00 & 67,74 & \\
\hline $100-200$ & 23,57 & 15,04 & 0,00 & 48,39 & \\
\hline \multirow[t]{2}{*}{$<100$} & 26,34 & 15,26 & 0,00 & 58,06 & \\
\hline & & & & & 0.279055 \\
\hline 7 Kommunikator & MW & SD & Min. & Max. & $\mathbf{p}$ \\
\hline Uniklinik & 52,38 & 26,87 & 16,67 & 100,00 & \\
\hline$>200$ & 41,30 & 29,02 & 0,00 & 100,00 & \\
\hline $100-200$ & 50,00 & 28,99 & 0,00 & 83,33 & \\
\hline \multirow[t]{2}{*}{$<100$} & 49,31 & 28,55 & 0,00 & 100,00 & \\
\hline & & & & & 0.616112 \\
\hline
\end{tabular}




\begin{tabular}{|c|c|c|c|c|c|}
\hline 8 Mitglied eines Teams & MW & SD & Min. & Max. & $\mathbf{p}$ \\
\hline Uniklinik & 45,28 & 20,43 & 12,50 & 78,26 & \\
\hline$>200$ & 32,40 & 18,28 & 0,00 & 78,26 & \\
\hline $100-200$ & 37,70 & 19,70 & 4,17 & 65,22 & \\
\hline \multirow[t]{2}{*}{$<100$} & 42,28 & 23,27 & 0,00 & 78,26 & \\
\hline & & & & & 0.172216 \\
\hline $\begin{array}{l}9 \text { Gesundheitsberater und - } \\
\text { fürsprecher }\end{array}$ & MW & SD & Min. & Max. & $\mathbf{p}$ \\
\hline Uniklinik & 40,74 & 21,02 & 5,56 & 88,89 & \\
\hline$>\mathbf{2 0 0}$ & 26,09 & 18,43 & 0,00 & 66,67 & \\
\hline $100-200$ & 23,50 & 18,12 & 5,56 & 61,11 & \\
\hline \multirow[t]{2}{*}{$<100$} & 38,89 & 28,41 & 0,00 & 88,89 & \\
\hline & & & & & 0.047644 \\
\hline $\begin{array}{l}10 \text { Verantwortungsträger und } \\
\text { Manager }\end{array}$ & MW & SD & Min. & Max. & $\mathbf{p}$ \\
\hline Uniklinik & 18,66 & 15,29 & 0,00 & 54,05 & \\
\hline$>200$ & 11,63 & 11,62 & 0,00 & 37,84 & \\
\hline $100-200$ & 16,01 & 10,99 & 0,00 & 37,84 & \\
\hline \multirow[t]{2}{*}{$<100$} & 17,34 & 11,91 & 0,00 & 43,24 & \\
\hline & & & & & 0.257188 \\
\hline 11 Professionell Handelnder & MW & SD & Min. & Max. & $\mathbf{p}$ \\
\hline Uniklinik & 34,33 & 17,45 & 10,42 & 70,83 & \\
\hline$>200$ & 22,19 & 17,23 & 0,00 & 62,50 & \\
\hline $100-200$ & 25,16 & 12,92 & 6,25 & 43,75 & \\
\hline \multirow[t]{2}{*}{$<100$} & 32,90 & 20,24 & 6,25 & 81,25 & \\
\hline & & & & & 0.112563 \\
\hline
\end{tabular}

Tab. 17 Erreichte Lernziele des Abschnitt 1 nach (Lehr-)Krankenhäusern. Mittelwerte \pm Standardabweichungen, Minima und Maxima dargestellt in \%.

In Bezug auf Abschnitt 2 lassen unsere Ergebnisse die Schlussfolgerung zu, dass im Mittel an Lehrkrankenhäusern mit unter 100 chirurgischen Betten (MW = 8,76 $\pm 6,90 \%$; Min. =0,00\%; Max. $=30,10 \%$ ) deutlich mehr Lernziele abgedeckt werden können. An der Universitätsklinik (MW = 6,93 $\pm 3,84 \%$; Min. = 1,85\%; Max. = $15,49 \%)$ können den Studierenden ähnlich viele oder leicht weniger Lernziele als im Durchschnitt vermittelt werden. An Lehrkrankenhäusern mit über 200 (MW = $5,57 \pm 3,71 \%$; Min. = 0,30\%; Max. = 13,12\%) und $100-200(M W=5,69 \pm 2,78 \%$; Min. $=2,38 \%$; Max. $=11,05 \%$ ) chirurgischen Bettenplätzen können die Studierenden im Mittel weniger Lernziele als im Gesamtdurchschnitt (6,88 $\pm 4,99 \%$; Min. $=0,00 \%$; Max. $=30,10 \%$ ) erlernen (Abb. 29). 


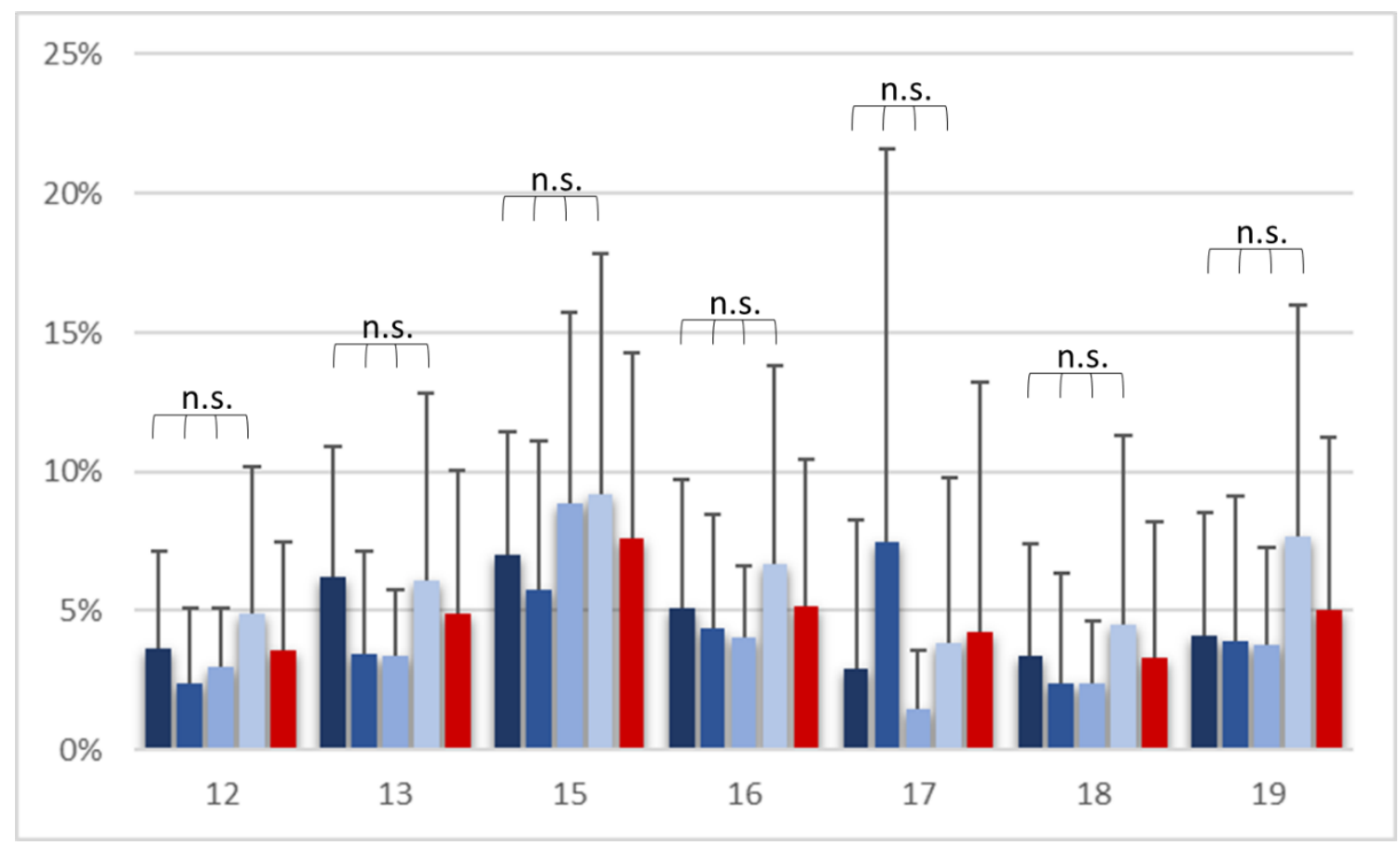

Abb. 29 Erreichte Lernziele des Abschnitt 2 (Kapitel 12, 13, 15 - 19) nach (Lehr-) Krankenhäusern. Mittelwerte + Standardabweichungen dargestellt in \%; von links nach rechts Uniklinik, >200, 100$200,<100$ und Gesamt. ${ }^{* *} p<0.01,{ }^{*} p<0.05$, n.s. $p>0.05$.

Besonders deutlich wird die Verteilung der im Blockpraktikum abdeckbaren Lernziele in den Kapiteln 14 a - c. Diese Kapitel beinhalten die Lernziele der Medizinisch-wissenschaftliche Fertigkeiten (14a), Klinisch praktischen Fertigkeiten (14b) und der Ärztlichen Gesprächsführung (14c). In Lehrkrankenhäusern mit unter 100 chirurgischen Bettenplätzen, konnten Studierende im Blockpraktikum Chirurgie mehr klinisch-praktisch relevante Lernziele erreichen (Abb. 30). 


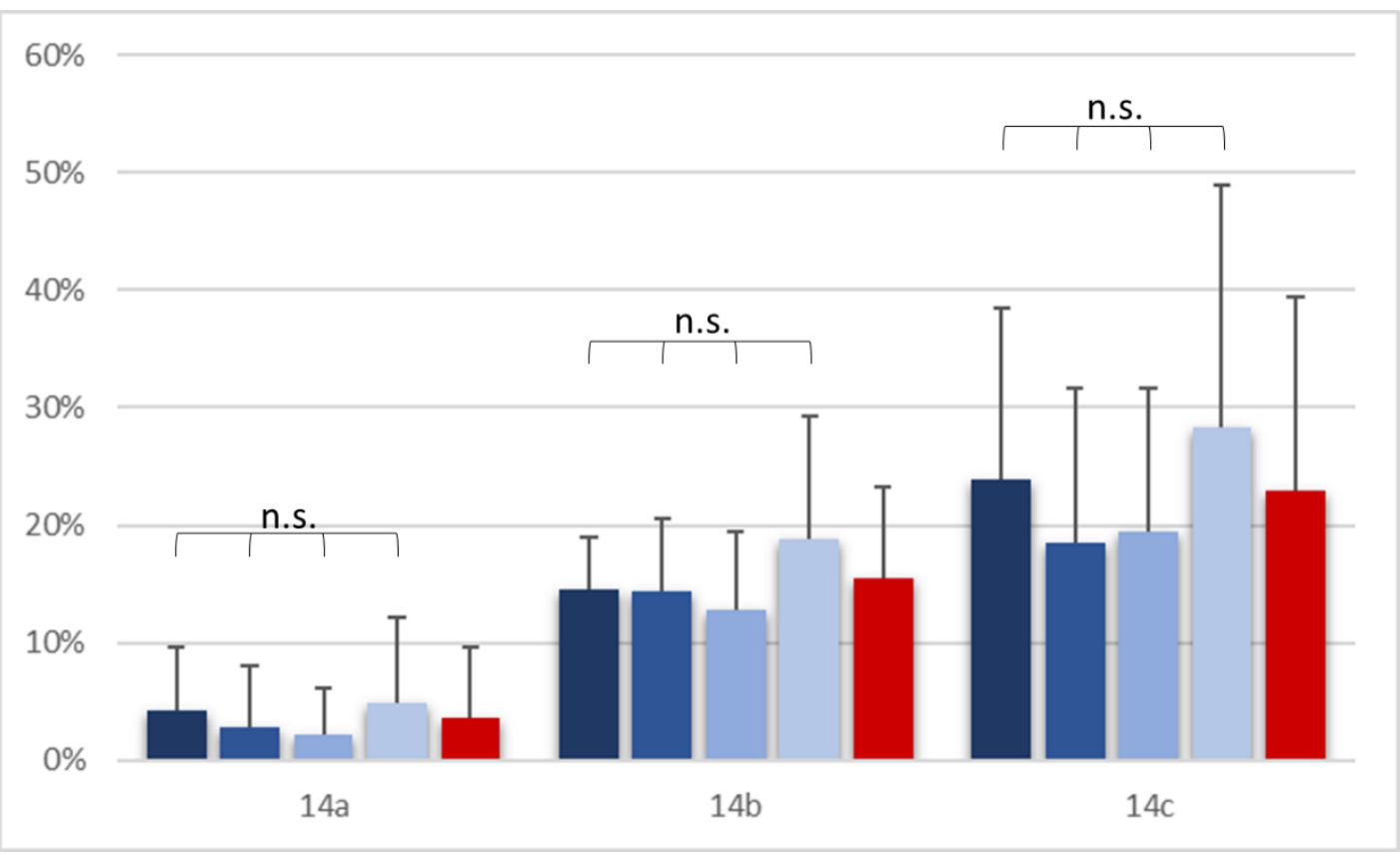

Abb. 30 Erreichte Lernziele des Abschnitt 2 (Kapitel 14 a - c) nach (Lehr-) Krankenhäusern. Mittelwerte + Standardabweichungen dargestellt in \%; von links nach rechts Uniklinik, >200, 100$200,<100$ und Gesamt. ${ }^{* *} p<0.01,{ }^{*} p<0.05$, n.s. $p>0.05$.

\begin{tabular}{|c|c|c|c|c|c|}
\hline 12 Physiologie & MW & SD & Min. & Max. & $\mathbf{p}$ \\
\hline Uniklinik & 3,63 & 3,48 & 0,00 & 12,90 & \\
\hline$>200$ & 2,41 & 2,68 & 0,00 & 9,25 & \\
\hline $100-200$ & 3,02 & 2,08 & 0,24 & 8,78 & \\
\hline \multirow[t]{2}{*}{$<100$} & 4,88 & 5,32 & 0,00 & 21,41 & \\
\hline & & & & & 0.330834 \\
\hline 13 Pathophysiologie & MW & SD & Min. & Max. & $\mathbf{p}$ \\
\hline Uniklinik & 6,20 & 4,72 & 0,67 & 14,09 & \\
\hline$>200$ & 3,44 & 3,68 & 0,00 & 14,09 & \\
\hline $100-200$ & 3,35 & 2,43 & 0,67 & 8,72 & \\
\hline \multirow[t]{2}{*}{$<100$} & 6,09 & 6,75 & 0,00 & 23,49 & \\
\hline & & & & & 0.216976 \\
\hline $\begin{array}{l}\text { 14a Medizinisch-wissenschaftliche } \\
\text { Fertigkeiten }\end{array}$ & MW & SD & Min. & Max. & $\mathbf{p}$ \\
\hline Uniklinik & 4,19 & 5,52 & 0,00 & 21,05 & \\
\hline$>200$ & 2,79 & 5,24 & 0,00 & 21,62 & \\
\hline $100-200$ & 2,25 & 3,90 & 0,00 & 13,16 & \\
\hline \multirow[t]{2}{*}{$<100$} & 4,86 & 7,34 & 0,00 & 24,32 & \\
\hline & & & & & 0.562952 \\
\hline 14b Klinsch-praktische Fertigkeiten & MW & SD & Min. & Max. & $\mathbf{P}$ \\
\hline Uniklinik & 14,57 & 4,43 & 7,61 & 27,17 & \\
\hline
\end{tabular}




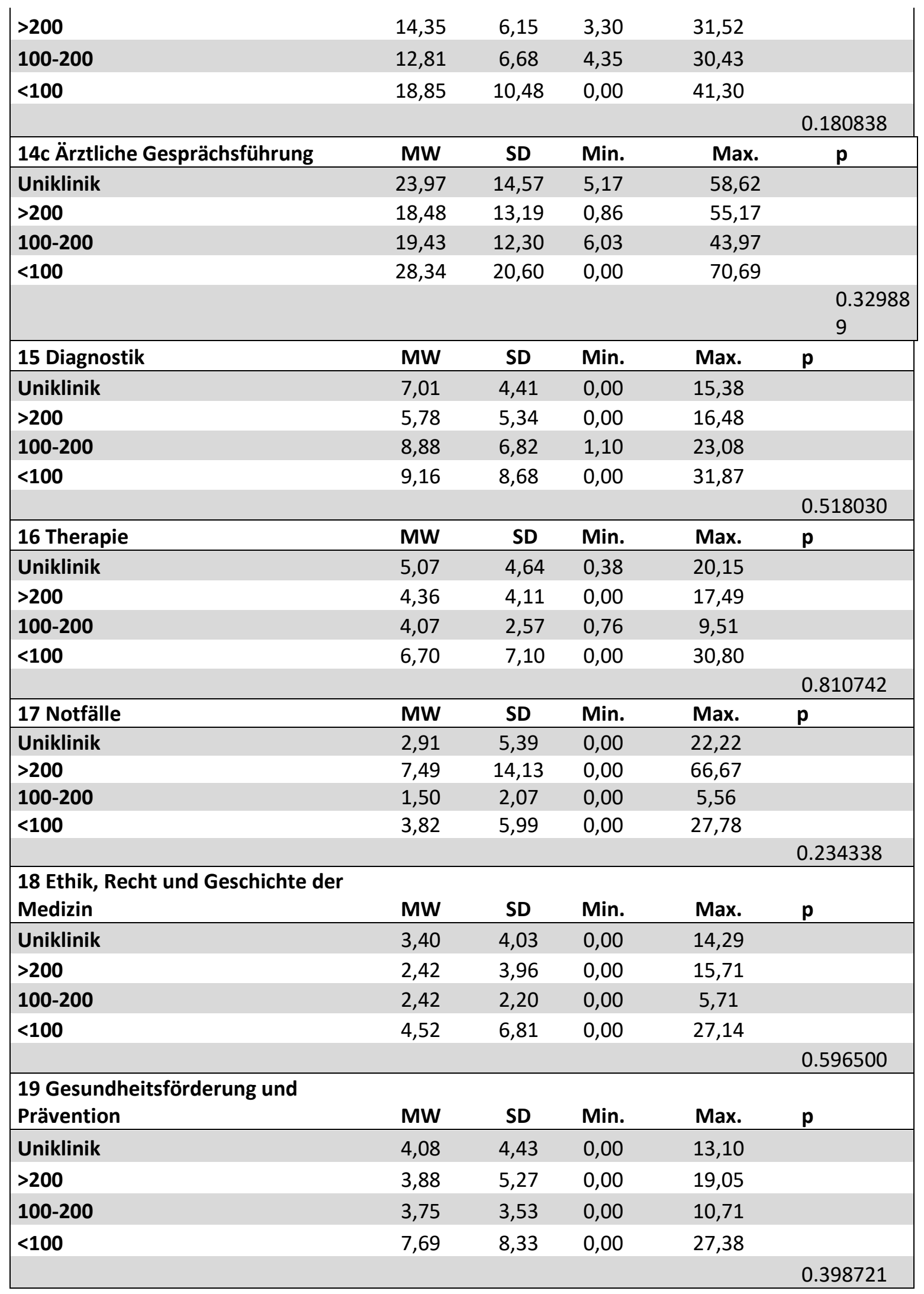


Tab. 18 Erreichte Lernziele des Abschnitt 2 nach (Lehr-)Krankenhäusern. Mittelwerte \pm Standardabweichungen, Minima und Maxima dargestellt in \%.

In Abschnitt 3 des NKLM können unsere Ergebnisse zeigen, dass die Lernziele durch die einzelnen Lehrkrankenhaus-Kategorien relativ homogen abgedeckt werden können. Allerdings zeigt sich auch hier wieder die Tendenz, dass Studierende in Krankenhäusern mit unter 100 chirurgischen Bettenplätzen mehr Anlässe ärztlicher Konsultation (Kapitel 20) oder Krankheitsbilder (Kapitel 21) in ihrem Blockpraktikum Chirurgie als Lernziel vermittelt bekommen konnten (Abb. 31).

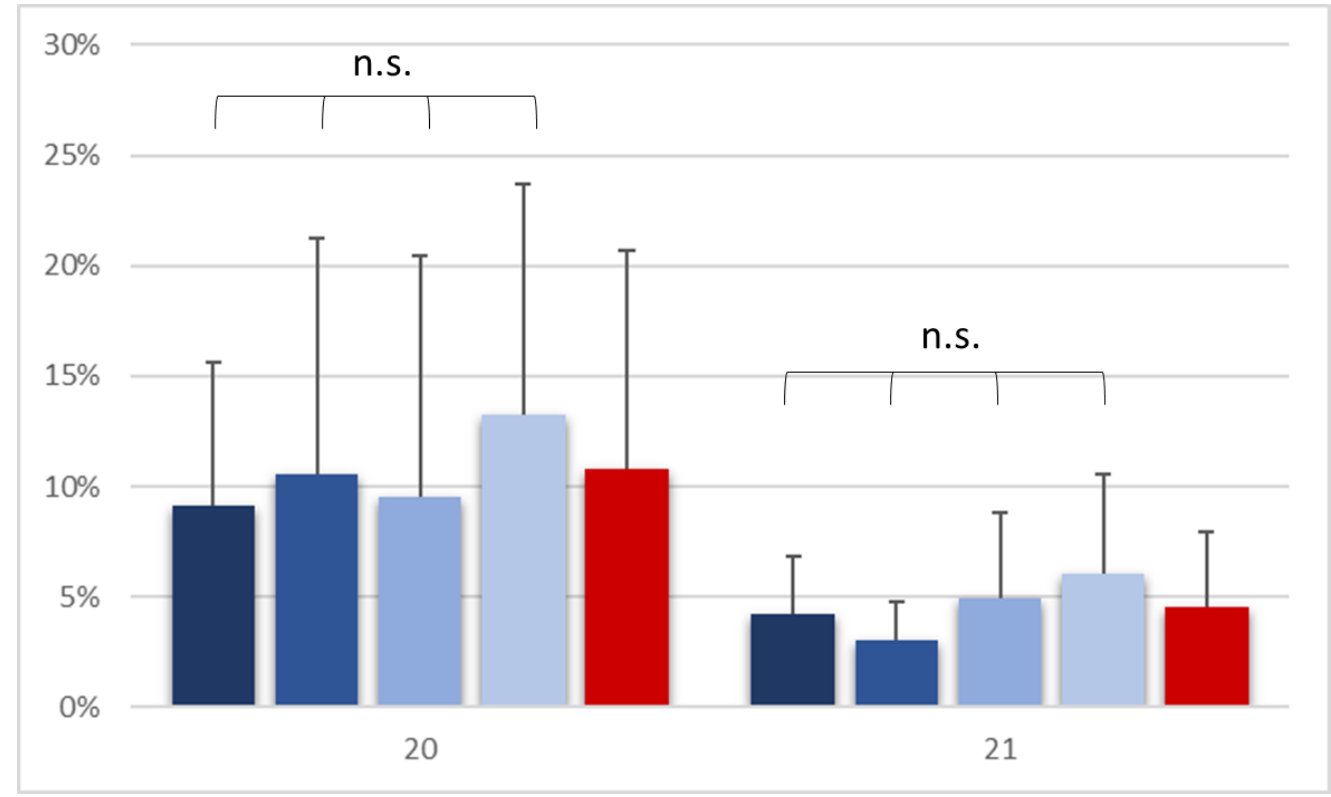

Abb. 31 Erreichte Lernziele des Abschnitt 3 nach (Lehr-)Krankenhäusern. Mittelwerte + Standardabweichungen dargestellt in \%; von links nach rechts Uniklinik, >200, 100-200, <100 und Gesamt. ${ }^{* *} p<0.01,{ }^{*} p<0.05$, n.s. $p>0.05$.

\begin{tabular}{|c|c|c|c|c|c|}
\hline 20 Anlässe ärztlicher Konsultation & MW & SD & Min. & Max. & $\mathbf{p}$ \\
\hline Uniklinik & 9,10 & 6,55 & 0,00 & 24,19 & \\
\hline$>200$ & 10,52 & 10,74 & 0,00 & 51,61 & \\
\hline $100-200$ & 9,55 & 10,89 & 0,81 & 33,06 & \\
\hline \multirow[t]{2}{*}{$<100$} & 13,27 & 10,41 & 0,00 & 41,94 & \\
\hline & & & & & 0.324667 \\
\hline $\begin{array}{l}21 \text { Erkrankungsbezogene Prävention, } \\
\text { Diagnostik, Therapie, Versorgungs- } \\
\text { und Notfallmaßnahmen }\end{array}$ & MW & SD & Min. & Max. & $\mathbf{p}$ \\
\hline Uniklinik & 4,18 & 2,67 & 0,68 & 10,88 & \\
\hline$>200$ & 3,04 & 1,76 & 0,23 & 6,58 & \\
\hline $100-200$ & 4,91 & 3,87 & 2,04 & 13,61 & \\
\hline
\end{tabular}


$<100$

6,01

4,51

0,45

16,78

Tab. 19 Erreichten Lernziele in Abschnitt 3 nach (Lehr-)Krankenhäusern. Mittelwerte \pm Standardabweichungen, Minima und Maxima dargestellt in \%.

\subsection{Benötigte Ressourcen}

Den Studierenden wurde ein kurzfristiger HiWi-Vertrag erstellt und für die Studienteilnahme 5 Zeitstunden mit 15,00€/h ausbezahlt. Dem betreuenden HiWi wurden in diesem Projekt 55 Stunden zu 15,00€/h vergütet. Für die Nutzung des Survey-Monkey Programms wurde eine einmalige Zahlung von 600,00€ veranschlagt. Die Erstellung des Online-Fragebogens im Survey-Monkey wurde von einem externen Mitarbeiter durchgeführt und mit 16 Stunden zu 15,00€/h abgerechnet. Somit ergibt sich eine Gesamtsumme von $7740 €$. 


\section{Diskussion}

\subsection{Allgemeines}

\subsubsection{Arbeitsaufwand Mapping}

Die Erstellung und Weiterentwicklung von kompetenzbasierten Curricula ist ein zentraler Bestandteil gut strukturierter und nachhaltiger Lehre. ${ }^{57,61,74}$ Mit dem Mapping des Blockpraktikums Chirurgie an der Goethe Universität Frankfurt am Main ist uns eine übersichtliche Kartierung der dort erreichten Lernziele gelungen. Wir können somit zeigen, welche Lernziele von rein wissensbedingten Kategorien bis hin zu praktischen und kommunikativen Fertigkeiten in einem klinischen Praktikum vermittelt werden können. Ein Mapping Prozess ist im gesamten an vielen Stellen mit einem hohen Ressourcenaufwand verbunden, was Zeit, Kosten und Personal betrifft. ${ }^{71}$ Auch die Programmbeschaffenheit hat Einfluss auf den Erfolg und die Durchführung des Mapping. ${ }^{63,65}$ Mit dem Tübinger MERLIN Projekt ist eine web-basierte Datenbank-Anwendung erstellt worden, durch die der MappingProzess gut gelingt. ${ }^{78}$ Zur effektiven Durchführung des Mapping unseres BP Chirurgie konnten wir mit einem Online-basierten Fragebogen ein Medium schaffen, das sich auch für den ungeübten Anwender gut bearbeiten lässt. So konnten wir sicherstellen, dass die Studienteilnehmer ihre Selbsteinschätzungen eindeutig und einheitlich festhalten konnten. Der zeitliche Aufwand von im Mittel 2h 41 min zur Bearbeitung war insgesamt moderat.

Trotzdem kann die Umsetzung eines Mapping Projekts generell, sowie auch unsere Umsetzung durchaus als Limitation angesehen werden. Die Studienteilnehmer hatten Aussagen über sehr viele Lernziele zu treffen und waren sowohl mit dem Konzept des NKLM als auch mit den Grundprinzipien der CanMEDS Rollen wenig bis gar nicht vertraut. Um eine korrekte und konkrete Bearbeitung zu gewährleisten, haben wir die Abfrage mittels Online-Bogen vereinfacht und auf eine Zuordnung von Kompetenzebenen verzichtet. Zudem fand vor der Bearbeitung eine Einführung durch einen Mapping-erfahrenen HiWi statt. Dieser stand für jegliche Rückfragen während der gesamten Bearbeitungszeit den Studienteilnehmern zur Verfügung. 


\subsubsection{CanMEDS}

In der Gesamtauswertung unserer Studie konnten wir deutlich zeigen, dass der Abschnitt der "Ärztlichen Rollen“ am stärksten in den im BP Chirurgie erreichten Lernzielen repräsentiert ist. Das betont die Wichtigkeit des ärztlichen Rollenverständnisses und die Relevanz deren Anwendung in der klinischen Praxis. Wir konnten zeigen, dass unsere Studierenden am meisten Lernziele aus den Kapiteln mit den Rollen „Kommunikator“, „Medizinischer Experte“ und „Mitglied eines Teams" erlernen konnten. Damit können relevante ärztliche Fertigkeiten bereits in einer frühen Phase der klinischen Ausbildung an die Studierenden vermittelt werden. Das schafft eine Grundlage, das erworbene Wissen im weiteren Verlauf der Ausbildung zu vertiefen und deren Anwendung in die klinische Arbeitsroutine zu integrieren.

\subsubsection{Klinische Relevanz und Rollenverständnis}

Die Gewichtung dieser Rollen konnten auch in mehreren Studien, z.B von Griewatz et al. 2016, Bugaj et al. 2017, Stutsky et al. 2012 und Jilg et al. 2015 gezeigt werden. 59,60,62,87 Für Studierende im PJ gelten in diesen Studien „Kommunikator“ (42\%), „Professionell Handelnder" (38\%) und „Mitglied eines Teams“ (7\%) als die wichtigsten 3 der CanMEDS Rollen. ${ }^{87}$ Lehrende bemessen die größte Wichtigkeit für die Rolle "Medizinischer Experte". Entsprechend sind die Rollen des „Kommunikator" und "Mitglied eines Teams" ähnlich wichtig bewertet. "Gesundheitsfürsprecher" und "Gelehrter" empfinden Lehrende als weniger wichtig. ${ }^{62}$ Wichtig sind auch die Ergebnisse dieser Arbeit für das Verständnis des Rollenkonzepts. So konnte gezeigt werden, dass auch ohne große Vorkenntnis, die Rollen des Medizinischen Experten, Kommunikator und Teammitglied gut interpretiert und verstanden werden, die des Gesundheitsfürsprechers und Gelehrten eher schlecht. ${ }^{62}$ Die Aspekte der Rollen, die von unseren CanMEDSnaiven Studierenden als am meisten gelernt bewertet worden sind, entsprechen also auch den Rollen, die das gängigste Rollenverständnis aufweisen. Die Betonung der genannten Rollen für die klinischen Praxis stellt auch die Arbeit von Jilg et al. 2015 und Stutsky et al 2012 dar. Hier konnte gezeigt werden, dass 
Klinikärzte alle CanMEDS Rollen für ihren Klinikalltag als relevant einstufen. Hierbei werden die Rollen „Kommunikator“, „Medizinischer Experte“ und „Mitglied eines Teams" besonders hervorgehoben. ${ }^{59}$ Weiterhin konnte dabei gezeigt werden, dass sich keine Unterschiede zwischen den Fächern Innere Medizin und Chirurgie, sowie zwischen der Universitätsklinik und akademischen Lehrkrankenhäusern ergeben. ${ }^{60}$ An dieser Stelle widersprechen unsere Ergebnisse jedoch dieser These. Wir konnten zeigen, dass die Lernziele der ärztlichen Rollen an den kleineren Lehrkrankenhäusern mit unter 100 chirurgischen Bettenplätzen und an der Universitätsklinik häufiger vermittelt werden können. Die Einbettung des CanMEDS Rollenkonzepts in Unterrichtsformen, klinische Lehre und den klinischen Arbeitsalltag ist ein zentrales Ziel, um eine kompetenzorientierte Aus- und Weiterbildung von Ärzten zu gewährleisten.

Wir konnten in unserer Studie zeigen, dass diese bereits Teil der chirurgischpraktischen Ausbildung an der Goethe Universität sind, ohne dass diese im Lernzielkatalog der Universität verankert sind und ohne eine konkrete Lehrmethode zu benennen. Die Vermittlung erfolgte für unsere Studierende überwiegend implizit. Dass die implizite Vermittlung von Lernzielen und grade die der CanMEDS Rollen eine effektive Methode ist, zeigen Turner et al. 2012. Sie beschreiben in einem „near-peer shadowing“-Programm, dass es kostengünstig möglich ist, Studierenden im ersten Jahr das CanMEDS Rollenkonzept zu vermitteln, indem sie Assistenzärzte im ersten Jahr begleiteten. Studienteilnehmer berichten dort, so ein ausführliches Verständnis der ärztlichen Rollen erlangt zu haben. ${ }^{88}$ Sie sollen fest in der Umsetzung des PJ Unterrichts verankert werden, ${ }^{60}$ aber auch im klinischen Alltag Anwendung finden und trainiert werden. Hassan et al. 2015 beschreibt, dass CanMEDS Rollen zwar mit größerem Zeitaufwand detailliert z.B. in RoutineBesprechungen angewendet werden, aber sinnvoll für die Diskussion komplexer Fragestellungen in Journal clubs oder critical case reports/ethical dilemma sind. ${ }^{89}$ Dass in der chirurgischen Weiterbildung sowohl chirurgische Skills als auch CanMEDS Rollen gleichzeitig vermittelt werden, zeigen Ponton-Carss et al. 2014.90 Die Aufnahme des ärztlichen Rollenkonzepts in Anlehnung an die CanMEDS Rollen 
in den Lernzielkatalog Chirurgie des Fachbereich 16 der Goethe Universität Frankfurt scheint somit durchaus sinnvoll.

\subsubsection{Anwendung der CanMEDS in Prüfungen}

Die Angaben zu den erreichten Lernzielen der Teilnehmer unserer Studie beruhen auf deren Selbsteinschätzung. Sie bilden somit das subjektiv „Learned curriculum“ ab, sind aber nicht objektiv abgeprüft worden. So muss man als weitere Limitation dieser Ergebnisse sehen, dass die Kartierung der erreichten Lernziele im Blockpraktikum Chirurgie nicht auf Prüfungsergebnissen beruht, sondern auf dem Selbstverständnis der Studierenden. Die Objektivierung eines auf Selbsteinschätzung von Studierenden beruhenden "Learned Curriculum" hat Zelenitsky et al. 2014 vorgenommen, indem die späteren Arbeitgeber der Absolventen $\mathrm{zu}$ deren Fähigkeiten und Fertigkeiten befragt wurden. ${ }^{69}$ Wie lässt sich aber in standardisierten Prüfungen bereits während des Studiums feststellen, ob die von den Studierenden angegebenen Lernziele der Ärztlichen Rollen tatsächlich gelernt wurden? Aufgrund der eben verdeutlichten Relevanz dieser für den klinischen Arbeitsalltag und das ärztliche Rollenverständnis, sollten diese prüfbarer Inhalt der medizinischen Ausbildung sein. Die staatlichen Prüfungen mit den Prüfungsfragen des IMPP zeigen eine eher heterogene Abbildung der Inhalte des NKLM. So konnte eine Studie von Fritze et al. 2017 zeigen, dass es im Gegenstandskatalog zahlreiche Übereinstimmungen mit den NKLM Kapitel zu „klassischem Fachwissen (Kap. 12, 13, 16, 17 sowie 20 und 21)" und medizinisch-praktischen Fertigkeiten (Kapitel 14b) gibt. Eine Übereinstimmung für die mit der NKLM Erstellung 2015 neu formulierten Ärztlichen Rollen fand sich nicht. ${ }^{56}$ In den Abschlussprüfungen des M3 werden wenig Kompetenzorientierte Fragestellungen und kaum Fragen jenseits der Rolle des medizinischen Experten geprüft. ${ }^{91}$

Wie kann das Konzept der ärztlichen Rollen trotzdem Eingang in die Prüfungen der medizinischen Ausbildung erhalten? Eine probate Methode CanMEDS Rollen in einem Prüfungsformat zu testieren, ist die Durchführung eines OSCEs ${ }^{92}$ Vor allem die Rollen Kommunikator, Mitglied eines Teams, Professionell Handelnder und 
Manager lassen sich damit prüfen. ${ }^{93}$ Die Frankfurter Scheinvergabekriterien sehen vor, dass das Fach Chirurgie unter anderem mit einer bestandenen OSCE Prüfung abzuschließen ist, die sich in der Regel an das Ende des Semesters, in dem das Blockpraktikum Chirurgie absolviert wird, anschließt. Eine Alternative zur konstruierten OSCE Prüfung können Arbeitsplatzbasierte Prüfungen sein. Dabei wird eine Prüfung in einem realen, klinischen Setting abgehalten. Studien konnten zeigen, dass arbeitsplatzbasierte Prüfungen und Lehren ein valides und wichtiges Instrument sind, um Feedback zu ermöglichen und praktische Fertigkeiten zu erlernen. ${ }^{94,95}$ Eine Untersuchung der LMU München konnte 2014 zeigen, dass sich dieses Prüfungsformat eignet, um klinisch praktische Fertigkeiten im Blockpraktikum Allgemeinmedizin zu prüfen. Es handelt sich dabei um eine Prüfung formativen Formats. ${ }^{96}$ Implementiert man arbeitsplatzbasiertes Lernen in der medizinischen Ausbildung, vermittelt den Studierenden Feedback und überprüft man deren Fertigkeiten in einem OSCE, so kann dann die Selbsteinschätzung der Teilnehmer verifizieren. Ein auf Erfahrungswerten basierendes „Learned Curriculum“ kann dadurch objektiviert werden.

\subsubsection{Wichtigkeit der klinisch-praktischen Ausbildung}

Die praktisch-klinisch Ausbildung ist ein zentraler Bestandteil des Medizinstudiums ${ }^{1}$ und von großer Bedeutung für Studierende..$^{97,98}$

Das spiegelt sich auch in unseren Ergebnissen der Kapitel 14 a -c wider. So geben Studierende an, einen vergleichsweise hohen Anteil der in diesen Kapiteln formulierten Lernziele im Blockpraktikum Chirurgie erlernt zu haben. Die Kapitel beinhalten mit den klinisch-praktische Fertigkeiten und der Ärztlichen Gesprächsführung wichtige Instrumente des klinischen Altags als Arzt. Die Lernziele aus Kapitel 15 und 16 zu Diagnostischen und Therapeutischen Prinzipen werden nach unseren Ergebnissen im Blockpraktikum Chirurgie deutlich weniger vermittelt. Unstrittig ist, das Kenntnis zu Diagnostik und Therapie unabdingbar für die Anforderungen als Arzt im klinischen Alltag sind. Das unterstützt die Annahme, dass Studierende ihre Ausbildung in Praktika und Famulaturen als nicht genügend empfinden. ${ }^{97-99}$ Aber, alleinige Stationspraktikum reichen anscheinend zum Erlernen 
praktischer Fertigkeiten nicht aus. ${ }^{100,101}$ Es kommt häufig zu Frustration im Rahmen von Praktika und Erwartungen nicht erfüllt werden, da Studierende gerade zu Beginn der klinischen Ausbildung ihren eigenen Ausbildungsstand und ihre Kompetenzen in den verschiedenen Fertigkeiten nicht genügend einschätzen können und selten Rückmeldung dazu erhalten. ${ }^{102}$ Eine Möglichkeit Studierende besser auf das Erlernen und Einschätzen von klinischen Fertigkeiten in Praktika vorzubereiten, stellt die von Bornemann et al. 2014 vorgestellte Methode dar, ein Zertifikat zur „Famulaturreife" auszustellen. ${ }^{103}$ So könnten auch Lehrende an Krankenhäusern besser den Ausbildungsstand der Praktikumsteilnehmer einschätzen und die Studierenden entsprechend in den klinischen Alltag mit einbinden. Die medizinische Ausbildung muss so umfangreich gestaltet sein, dass sie auf den Klinikalltag als Arzt vorbereitet. Viele Berufsanfänger fühlen sich nach dem Studium inadäquat auf den Berufseinstieg vorbereitet ${ }^{104}$ und empfinden ihren neuen Arbeitsalltag als stressvoll. ${ }^{105}$ (Brennan, Corrigan et al. 2010) Für die Chirurgie und die Urologie konnte Stefanescu et al. 2018 zeigen, dass sich nur 43\% der Assistenzärzte ausreichend auf ihren Berufsstart vorbereitet fühlen und ebenso die ersten Wochen als stressig empfinden. ${ }^{5}$

\subsection{Geschlechterunterschied}

\subsubsection{Selbstwirksamkeit Männer und Frauen + Effekt auf weitere Karriere}

Dass Frauen im Medizinstudium und in der ärztlichen Tätigkeit immer stärker repräsentiert sind, zeigt die Statistik. Derzeit sind $62,5 \%$ der Humanmedizinstudierenden weiblich. ${ }^{106}$ Die Ärztezahl in Deutschland steigt generell an, sowie auch die Anzahl der Frauen im Arztberuf. Betrachtet man den Bereich der Chirurgie steigt auch hier der Anteil der Frauen an (Zeitraum 2015-2019). ${ }^{107} \mathrm{Im}$ Gegensatz dazu sehen Männer sich nach dem Studium eher in den Chirurgischen Fachdisziplinen mit Schwerpunktsetzung als Frauen. ${ }^{108}$ Wir konnten zeigen, dass Männer angeben im chirurgischen Blockpraktikum, im Gesamten mehr zu lernen als Frauen. Vor allem in Abschnitt 1 der Ärztlichen Rollen sind die Unterschiede deutlich. Auch für das Kapitel 14b der klinisch-praktischen Fähigkeiten gilt das. Unterschiede werden weniger deutlich, wenn es um die Anzahl der im Blockpraktikum Chirurgie 
erlebten ärztlichen Konsultationsanlässe geht. In keinem der Kapitel geben Frauen an mehr gelernt zu haben als Männer. Kausale Zusammenhänge können wir aus unseren Ergebnissen nicht ableiten. Die Geschlechtszugehörigkeit per se scheint aber jedoch eine Bedeutung für den subjektiven Lernerfolg zu haben. Frauen betonen häufiger, dass die Geschlechterzugehörigkeit in medizinischen Bereichen, unter anderem in der klinischen Lehre, von Bedeutung ist als Männer. Dies ist unabhängig von Spezialisierung, Alter, akademischem Grad und Jahren an Berufserfahrung festzustellen. ${ }^{109}$ Den Einfluss des Geschlechts können auch Ergebnisse von Studien aus anderen Berufsbereichen feststellen. So konnte eine Studie von Ro von 2016 in Ingenieurstudiengängen zeigen, dass Frauen angeben, über weniger Fähigkeiten zu verfügen als Männer. Das gilt vor allem für Fähigkeiten wie Führungskraft, Teamwork. Ihre kommunikativen Fähigkeiten schätzen sie höher ein. Die Gründe dafür bleiben auch in dieser Studie unklar. ${ }^{110}$ Diese These unterstreichen auch Kalender et al. 2018, indem sie zeigen konnten, dass Frauen in Bereichen, in denen sie unterrepräsentiert sind bei gleichem Ausbildungs- und Prüfungsstand eine geringere Selbstwirksamkeit als Männer angeben. ${ }^{111}$ Eine geringere Selbstwirksamkeit bei Frauen könnte an dieser Stelle der Grund sein, weshalb Männer angeben, mehr zu lernen als Frauen, denn Vorbereitung und Selbstwirksamkeit haben einen Einfluss auf das Lernergebnis. ${ }^{112} \mathrm{Im}$ übertragenen Sinne für den medizinischen Bereich konnte Zimmerman et al. schon 2000 zeigen, dass eine geringere Selbstwirksamkeit das Interesse für das jeweilige Fach schmälern kann. ${ }^{113}$ Somit scheint es auch deutlich zu sein, dass die Selbstwirksamkeit in Zusammenhang mit dem Lernerfolg den weiteren Karriereverlauf beeinflusst. Gerade Bereiche wie im angloamerikanischen Raum „Science Technology Engineering Mathematics“ (STEM) bzw. im Deutschen die MINT Fächer („Mathematik, Informatik, Naturwissenschaft, Technik“), in denen Frauen lange unterrepräsentiert waren und es tendenziell noch sind, sind die Effekt der Selbstwirksamkeit gut untersucht. So konnte schon vor Jahrzehnten die Arbeit von Seymour und Hewitt zeigen, dass der Karriereverlauf bei Frauen in STEM Bereichen von den unterschiedlichsten Faktoren aber vor allem vom Lernerfolg abhängig ist. Ein fehlender Lernerfolg führte bei Frauen häufiger dazu, dass sie eine 
weitere Karriere in STEM Bereichen aufgeben, als Männern. ${ }^{114}$ An dieser Stelle kann nochmal die Wichtigkeit von klinisch-praktischer Prüfungen betont werden, um die Selbsteinschätzung zu objektivieren. Wenn Frauen feststellen, dass ihre Prüfungsergebnisse $z u$ entsprechenden Fähigkeiten besser ausfallen als ihre Selbsteinschätzung, dann könnte es dazu verhelfen, dass das Interesse an dem jeweiligen Fach nicht schwindet. Zudem könnte ein besseres Prüfungsergebnis als die Selbsteinschätzung als motivierender Faktor angesehen werden, sich mehr mit dem jeweiligen Fach auseinander zu setzen und die Fähigkeiten zu vertiefen. Denn im Gegensatz zur These, dass dem selbst eingeschätzten Lernerfolg in der Chirurgie eine Geschlechterabhängigkeit anhaftet, zeigt eine Erhebung von Lindlohr et al. 2011, dass die Form der Lernmethode der einflussreichste Faktor für den Lernerfolg ist. Der Lernerfolg bezog sich hier auf ein Operationsergebnis am Trainingsmodell. Es konnte gezeigt werden, dass das Geschlecht generell keine Rolle in Bezug auf das Operationsergebnis spielt. ${ }^{115}$

\subsection{2 Über-/Unterschätzer}

Neben dem Faktor Motivation spielt auch die realistische Einschätzung der eigenen Fähigkeiten eine wichtige Rolle. Sowohl überschätzen als auch unterschätzen der eigenen Fähigkeiten bilden ein Risiko für die Patientensicherheit und für die kollegiale Zusammenarbeit. Überschätzung kann dazu führen, dass manche (Notfall-) Situationen nicht erkannt und beherrscht werden oder zu spät Hilfe angefordert wird. Das kann sowohl für mangelndes Wissen, aber auch für nicht beherrschte Fertigkeiten gelten. Auf der anderen Seite kann ein Unterschätzen der eigenen Fähigkeiten auch Gefahr für Fehler bieten, wenn Entscheidungen durch Unsicherheit verzögert werden oder Maßnahmen nicht sicher und zügig durchgeführt werden. Schon 1991 diskutierten Jankowski et al. ihre Ergebnisse, dass Ärzte aller Weiterbildungsstufen ihre Fähigkeiten und ihr Wissen sowohl überals auch unterschätzen, in Bezug auf die klinische Auswirkung und die Patientensicherheit. Dabei kommt auch diese Arbeit zu dem Schluss, dass fehlerhafte Selbsteinschätzung im klinischen Kontext potenziell gefährlich ist. ${ }^{116}$ Eine aktuelle Studie aus dem chirurgischen Kontext von Borracci et al. 2019 konnte 
zeigen, dass vor allem in frühen Weiterbildungsstufen die Einschätzung der eigenen Fähigkeiten für Assistenzärzte ein Problem darstellen kann. So beschreiben die Ergebnisse, dass junge Ärzte in Trainings für chirurgische Fähigkeiten sich häufig besser einschätzen als es einer klassischen Lernkurve und der Einschätzung eines erfahrenen Chirurgen entspräche. Ein Feedback könne aber Selbsteinschätzung der jungen Kollegen verbessern. ${ }^{117}$

Im übertragenen Sinne für das Medizinstudium konnte dargestellt werden, dass gerade in frühen Ausbildungsabschnitten Studierende besonders anfällig sind, ihre Performance nicht adäquat einschätzen zu können. Im weiteren Verlauf des Studiums gelingt dies besser. Dabei unterschätzen sich weibliche Studierende eher als männliche Studierende. ${ }^{118}$ Das Unterschätzen der eigenen Fähigkeiten und des eigenen Wissen kann ebenso ein Grund sein, weshalb die Ergebnisse unserer Studie zeigen, dass Frauen angeben weniger Lernziele erreicht zu haben. In so hochsensiblen Bereichen der Patientenversorgung müssen die Patientensicherheit und der Qualitätsanspruch immer gewährleistet bleiben, die praktische Ausbildung von Studierenden und Assistenzärzten aber trotzdem adäquat integrierbar sein. Czeskleba et al. konnten 2019 zeigen, dass Ärzte, die PJStudenten betreuen, in deren Einbindung in die Arbeit mit Patienten ein (indirektes) Risiko für die Patientensicherheit auf unterschiedlichsten Ebenen sehen. ${ }^{119}$ Für den Umgang mit Studierenden und deren Einbeziehen in die Patientenversorgung kann eine Möglichkeit die Formulierung von „Anvertraubaren Professionellen Tätigkeiten“ (APT bzw. englisch Entrustred Professional Activities = EPA) sein. So kann sowohl für den betreuenden Arzt, der die Verantwortung überträgt als auch für den Studierenden, der mit dieser Verantwortung umgeht, ein sicheres Umfeld geschaffen werden. Fragen wie: „Was kann ich meinen Studierenden zutrauen, welche Fertigkeiten beherrschen sie tatsächlich?" oder „Welche Fähigkeiten werden von mir im Praktikum erwartet, welche Fertigkeiten muss ich noch vertiefen, um sie sicher anwenden zu können?" können so transparenter gestaltet werden. Um dieses Sicherheitsrisiko zu minimieren und Studierende sicherer auszubilden, ist eine Formulierung von APT je nach Ausbildungsstand wichtig. Vorschläge zur Formulierung von APT im Praktischen Jahr existieren bereits. ${ }^{120}$ Eine Erarbeitung 
von APT in klinischen Praktika für Studierenden im klinischen Studienabschnitt erscheinen an dieser Stelle sinnvoll. Die Patientensicherheit und die Qualität der medizinischen Versorgung könnten so besser gewährleistet werden und der Lernerfolg sowie die realistische Selbsteinschätzung der eigenen Fertigkeiten der Studierenden verbessert werden, indem die Grad des Über- und Unterschätzens minimiert wird.

\subsection{Fachrichtungen}

\subsubsection{Chirurgie und praktische Fertigkeiten}

Aus unseren Ergebnissen geht hervor, dass bei Betrachtung der erreichten Lernziele in Bezug auf die unterschiedlichen chirurgischen Fachrichtungen Studierende, die über mehrere Stationen „Rotiert“ sind, angeben am meisten gelernt zu haben. Das betrifft in erster Linie die Kapitel der Abschnitte „Medizinisches Wissen, klinische Fähigkeiten und professionelle Haltungen“ und „Patientenzentrierte Gesundheitsversorgung“. Das lässt den Schluss zu, dass durch die Arbeit in unterschiedlichen Abteilungen ("Rotation") mehr Lernziele vermitteltet werden. Studierende können an einem breiteren Spektrum unterschiedlicher Operationen teilnehmen, lernen mehr Krankheitsbilder kennen und sehen mehr Prozeduren der jeweiligen Fachrichtung.

Im Frankfurter Blockpraktikum Chirurgie geben Studierende, die kleine chirurgische Fachabteilungen unter "Sonstige“ besucht haben an, mehr Lernziele der CanMEDS Rollen erreicht zu haben als andere Teilnehmer. Da Erhebungen von Rüsseler et al. 2017 zeigen konnten, dass Studierende den Großteil ihrer Praktikumszeit mit Assistenzärzten verbringen, ${ }^{102}$ legt dies nahe, dass sie die Lernziele der CanMEDS Rollen vermehrt von diesen vermittelt bekommen haben. Dass das Begleiten von Ärzten zum Erlernen ärztlicher Rollenkompetenzen eine Methode sein kann, zeigen wie schon erwähnt Turner et al. 2012. ${ }^{88} \mathrm{Im}$ Umkehrschluss stellt sich in unseren Ergebnissen dar, dass entsprechend weniger Lernziele der CanMEDS Rollen in den großen, spektrumreichen Fächern wie Unfallchirurgie und Allgemeinchirurgie erlernt werden können. Das lässt die Vermutung zu, dass bei vermehrter Arbeitsbelastung 
in großen Abteilungen und eine größere Anzahl an Blockpraktikanten pro Abteilung weniger Lernziele vermittelt werden können.

Auch hier konnten wir zeigen, dass Studierende in einzelnen Fachrichtungen wie Allgemeinchirurgie, Unfallchirurgie und kleinen Fächern unter "Sonstige“ weniger Lernziele der klinisch-praktischen Fertigkeiten und ärztlicher Gesprächsführung vermittelt bekommen. Primär lässt sich dazu auf der einen Seite festhalten, dass die Chirurgie an sich prädestiniert für die Vermittlung von Lernzielen nach einem schlüssigen Ausbildungskonzept ist, da alle Studierende diese in curricularen Veranstaltungen und im Pflichttertial des PJs durchlaufen. ${ }^{84}$ Der Ausbildung in praktischen Fertigkeiten und dem Erwerb praktischer klinischer Kompetenz kommt in allen Ausbildungsabschnitten eine große Bedeutung zu. Ein Blockpraktikum, wie es in dieser Studie untersucht worden ist, bietet den Studierenden die Möglichkeit, Basisfertigkeiten und erste klinische Algorithmen zu erlernen. Sie erhaschen zwar eher nur einen kleinen Einblick in das jeweilige Fach, das kann jedoch dazu führen, dass das Fach für sie attraktiver wird. ${ }^{102}$

\subsection{Lehrkrankenhäuser}

\subsubsection{Anforderungen an ein Lehrkrankenhaus}

Um die Einordnung unserer Ergebnisse in Bezug auf die Kategorien der Bettenkapazität der Lehrkrankenhäuser treffen zu können, bedarf es einer genaueren Betrachtung der Verteilung Studierender auf Uniklinikum oder Lehrkrankenhaus. Die Grundsätze der Vergabe von Blockpraktikumsplätzen gemäß Anzahl und Ort sind hochschulpolitisch und kapazitätsrechtlich hochkomplex. Die Verteilung regelt grundsätzlich das Dekanat des Fachbereichs. Regularien hierfür sind in der in der Kapazitätsverordnung Hessen ( $\$ 17$, Abs. 3 ) und dem hessischen Hochschulgesetz festgehalten und richten sich nach der patientenbezogenen jährlichen Aufnahmekapazität. Prinzipiell erfolgt die Zuteilung der Studierende für die Blockpraktika zunächst an die Universitätsklinik und je nach Bedarf werden die Kapazitäten der Lehrkrankenhäuser ausgeschöpft. Für Frankfurt ergibt sich insofern eine komfortablere Situation, als dass die Infrastruktur des Rhein-Main Gebiets viele Kliniken aufweist, die die Voraussetzungen (festgehalten in der ärztlichen 
Approbationsordnung) als akademisches Lehrkrankenhaus erfüllen. Für Universitätsstandorte in strukturschwächeren Regionen, gilt dieses Angebot nicht unbedingt. Sowohl für die Universitätsklinik als auch akademischen Lehrkrankenhäuser zahlt der Fachbereich pro Blockpraktikant eine, individuellen Kriterien entsprechende, Aufwandsentschädigung.

\subsubsection{Lehre im klinischen Alltag}

Die Ergebnisse dieser Studie stellen in Bezugnahme auf die Lehrkrankenhäuser dar, dass insgesamt am meisten Lernziele von den Studierenden erlernt worden sind, die ein Lehrkrankenhaus mit unter 100 chirurgischen Bettenplätzen besucht haben. Das zeigt sich sowohl in den Kapiteln des Abschnitts 2 („Medizinisches Wissen, klinische Fähigkeiten und professionelle Haltungen") als auch des Abschnitts 3 („Patientenzentrierte Gesundheitsversorgung“). Interessant zu betrachten sind die Kapitel 14b der Klinisch-praktischen Fertigkeiten und 14c der ärztlichen Gesprächsführung. An kleinen Krankenhäusern geben Studierende an am meisten Lernziele zu klinisch-praktischen Fertigkeiten und Ärztlicher Gesprächsführung erlernt zu haben. Im Einzelnen betrachtet ergibt sich, dass die Lernziele der CanMEDS Rollen in Abschnitt 1 des NKLM auch häufiger an Lehrkrankenhäusern mit unter 100 chirurgischen Bettenplätzen und dem Universitätsklinikum vermittelt werden können.

Eine Studie von Rüsseler et al. 2017 konnte bereits zeigen, dass sich große Diskrepanzen zwischen den einzelnen Lehrkrankenhäusern in Hinblick auf das ergeben, was die Studierenden lernen können und wie es gelehrt wird. Sie beschreiben, dass die unterschiedlichen Erlebnisse unter anderem abhängig von der Motivation und dem Interesse von Einzelpersonen. ${ }^{102}$ Gerade an der Universitätsklinik sehen sich Lehrende mit einer redundant hohen Anzahl an Praktikanten konfrontiert. Das kann dazu führen, dass mit dieser Flut immer wieder neuen, wechselnden Gesichtern die Motivation zur Vermittlung von Lernzielen nachlässt. Das wiederum führt auch zu einem Motivationsverlust der Studierenden, und wiederum unmotivierte Studierende werden nicht gerne beschult, ein Teufelskreis entsteht. Wenn weniger Studierende in kleineren Krankenhäusern 
ihr Blockpraktikum absolvieren, scheint das dazu zu führen mehr Lernziele zu erreichen.

Generell sind von anderen Autoren bereits Arbeiten veröffentlich worden, die die Integration von Lehre in den klinischen Altag beleuchten und deren Ergebnisse auch auf unsere Hypothesen Anwendung finden können. So beschreiben Sterz et al. 2016 in "Der Chirurg im Spagat - Lehre im klinischen Alltag" sehr genau die Hindernisse der Integration der Lehre in die klinische Arbeit. Dabei wird generell genannt, dass ein Personalmangel die Lehre im Klinischen Alltag behindere. Junge Assistenzärzte geben überwiegend fehlende Zeit und eigene Überforderung als Hindernis an. Eine weitere Erschwernis sei aber auch, dass Aufwand für Lehre zu wenig Anerkennung durch Vorgesetzte und Klinikleitung erfahre. Sowohl in peripheren Häusern (Lehrkrankenhäusern) als auch an der Universitätsklinik sei dies der Fall. ${ }^{121}$ Ähnliches beschreiben König et al. 2019. Sie beschreiben Zeitmanagement zur Bewältigung von Lehraufgaben in einem Spannungsfeld zur klinischen Tätigkeit. Darüber hinaus fühlten sich Lehrende durch die Gruppengröße der Studierenden im stationären Setting an guter Ausbildung gehindert. Personalmangel wurde auch hier genannt. Zudem nannten Assistenzärzte zu wenig eigene didaktische Kenntnisse fast doppelt so häufig wie Oberärzte. Lehre wird sowohl vom ärztlichen Personal als auch von Krankenpflegepersonal als zusätzliche Belastung angegeben. ${ }^{122}$ Auch Adili et al. 2013 beschreiben die wichtigsten Hindernisse für eine effektive Lehre und frühzeitige Übertragung von Kompetenzen auf jüngere Aus- und Weiterzubildende. Sie nennen zunehmenden ökonomischen Druck, Arbeitsverdichtung, gestiegene Ansprüche an die Patientensicherheit, zunehmende Spezialisierung der Kliniken auf wenige Krankheitsbilder und Zentrumsbildung, gestiegene Ansprüche von Patienten an die Behandlung durch einen „Spezialisten“, gestiegene Anforderungen und Ansprüche von Seiten der Lernenden an die Qualität der Aus- und Weiterbildung (Generation Y), Fehlen geeigneter Lehrinfrastruktur, Belastung und Störung der Routineabläufe und das Fehlen einer fundierten didaktischen Grundausbildung. ${ }^{35}$ Auf die Ergebnisse unserer Studie übertragen, kann sich sagen lassen, dass die Integration von Lehre in den klinischen Alltag trotz der beschriebenen Hindernisse besser in Lehrkrankenhäusern mit geringer Bettenkapazität gelingt, gemessen an 
den im BP erreichten Lernzielen. Das Blockpraktikum Chirurgie findet in einer sehr frühen Phase der klinisch praktischen Ausbildung statt und soll den Studierenden erste Kenntnisse und Basisfähigkeiten vermitteln. Tieferes Wissen und speziellere chirurgische Fähigkeiten kann und soll dieses Kurskonzept nicht vermitteln. Deshalb scheint es für den Lernerfolg der patientenzentrierten Gesundheitsversorgung und den klinisch-praktischen Fertigkeiten vollkommen ausreichend und sogar besser zu sein, wenn Studierende diesen Kurs an einem Lehrkrankenhaus mit einem geringeren, übersichtlicherem Basisspektrum absolvieren. 


\section{Schlussfolgerungen}

Aus den vorliegenden Untersuchungen können die folgenden Schlüsse gezogen werden:

Mit dem Mapping des Chirurgie Blockpraktikums anhand der Lernziele des NKLM ist eine erste curriculare Kartierung einer klinisch-praktischen Lehrveranstaltungen des Frankfurter Medizinstudiums gelungen.

Wir konnten zeigen, dass im Zeitraum des zweiwöchigen Kurs der Gesamtumfang des NKLM anteilig abdecken werden kann. Das Blockpraktikum bietet entsprechend einen ersten Einblick in das Fachgebiet der Chirurgie und vermittelt vor allem Lernziele des ärztlichen Rollenkonzepts, der ärztlichen Gesprächsführung und klinisch-praktische Fähigkeiten. Weniger vermittelt er diagnostische und therapeutische Prinzipien der Chirurgie.

Männer geben an, im Rahmen dieses Praktikums mehr gelernt zu haben als Frauen. Dies entspricht einer Selbsteinschätzung der erlernten Kompetenzen, eine objektive Überprüfung des Lernerfolgs hat nicht stattgefunden. Die Selbstwirksamkeit hat somit einen Einfluss auf den subjektiv erlebten Lernerfolg.

Das Fachgebiet der Chirurgie eignet sich gut zur Vermittlung unterschiedlichster Kompetenzen, die im NKLM festhalten sind. Sowohl Prinzipien der ärztlichen Rollen als auch klinisch-praktische Fertigkeiten werden vermittelt. Vor allem in kleineren chirurgischen Fachgebieten und in Lehrkrankenhäusern mit weniger chirurgischer Bettenkapazität konnten Studierende häufiger angeben entsprechende Lernziele erreicht zu haben. Der Umfang des Lernerfolgs hängt von der Integration der Lehre in den klinischen Alltag ab. Ein begrenzteres bzw. spezifischeres Spektrum chirurgischer Krankheitsbilder und eine geringere Patientenanzahl verringern nicht die Anzahl erlernter Lernziele. 


\section{Literaturverzeichnis}

1. Approbationsordnung für Ärzte vom 27. Juni 2002 (BGBI. I S. 2405), die zuletzt durch Artikel 3 des Gesetzes vom 16. März 2020 (BGBI. I S. 497) geändert worden ist

2. Hibbeler B, Medizinstudium: 4, 8 Bewerber pro Studienplatz. Dtsch Ärztebl. 2012;109(33-34).

3. Medizinischer Fakultätentag (MFT) der Bundesrepublik Deutschland e. V., Nationaler Kompetenzbasierter Lernzielkatalog Medizin (NKLM). 2012. http://www.nklm.de/kataloge/nklm/lernziel/uebersicht. Accessed December 29, 2020

4. Bundesvertretung der Medizinstudierenden in Deutschland e.V. (bvmd). Zukunft und Weiterentwicklung des Medizinstudiums. 2014.

5. Stefanescu M-C, Sterz J, Hoefer SH, Ruesseler M. Young surgeons' challenges at the start of their clinical residency: a semi-qualitative study. Innov Surg Sci. 2018;3(4):235-243.

6. Gerke W, Breipohl W, Forster J, et al. Medizinische Ausbildung und der Bologna-Prozess. GMS Z Med Ausbild. 2006;23(1):2006-2023.

7. Recker F, Riedel F, Stope M, Mustea A, Weiss M, Jennebach J. Der Masterplan Medizinstudium 2020 und die Implementierung des Nationalen Kompetenzbasierten Lernzielkatalogs Medizin - Chancen und Herausforderungen für die Gynäkologie und Geburtshilfe. GebFra. 2020;80.

8. Müller B. "Wir befinden uns in einer permanenten Reform (...)" - Reformen in der Medizinerausbildung zwischen 1989 bis 2009. Stuttgart: RobertBosch-Stiftung; 2012.

9. Wissenschaftsrat (WR) Neustrukturierung des Medizinstudiums und Änderung der Approbationsordnung für Ärzte; Empfehlungen der Expertenkommission zum Masterplan Medizinstudium 2020. Köln: Wissenschaftsrat; 2018. Available from: https://www.wissenschaftsrat.de/download/archiv/7271-18.pdf.

10. Frank JR, ed. The CanMEDS 2005 Physician Competency Framework. Better Standards. Better Physicians. Better Care. Ottawa, ON: Royal College of Physicians and Surgeons of Canada; 2005.

11. General Medical Council. Tomorrow's Doctors. Recommendations on Undergraduate Medical Education. London: GMC; 1993. 
12. Simpson J, Furnace J, Crosby J, et al. The Scottish doctor--learning outcomes for the medical undergraduate in Scotland: a foundation for competent and reflective practitioners. Med Teach. 2002;24(2):136-143.

13. Metz JC. 'Blueprint 1994': common objectives of medical education in The Netherlands. Neth J Med. 1999;55(4):165-167.

14. Bloch R, Bürgi H. The Swiss catalogue of learning objectives. Med Teach. 2002;24(2):144-150.

15. Putz R. Entwicklung der Studiengänge nach der neuen Approbationsordnung 2002. Vortrag auf MFT-Tagung Berlin. 2010;21.

16. Öchsner W, Forster J. Approbierte Ärzte-kompetente Ärzte? Die neue Approbationsordnung für Ärzte als Grundlage für kompetenzbasierte Curricula. GMS Z Med Ausbild. 2005;22(1):2005-2022.

17. Bundesministerium für Bildung und Forschung. Masterplan 2020. Berlin: Bundesministerium für Bildung und Forschung; 2017. Available from: https://www.bmbf.de/de/masterplan-medizinstudium-2020-4024.html.

18. Hautz SC, Hautz WE, Keller N, Feufel MA, Spies C. The scholar role in the National Competence Based Catalogues of Learning Objectives for Undergraduate Medical Education (NKLM) compared to other international frameworks. GMS Ger. Medical Sci. 2015;13.

19. Ruesseler M, Froehlich S, Ruesseler S, Zabel J, Sterz J, Obertacke U. Statuserhebung der studentischen Lehre in Orthopädie und Unfallchirurgie. Z Orthop Unfall. 2017;156:168-174.

20. Seifert LB, Hoefer SH, Flammiger S, et al. A nationwide survey of undergraduate training in oral and maxillofacial surgery. Oral Maxillofac Surg. 2018;22:289-296.

21. Mager RF. Lernziele und programmierter Unterricht. Weinheim: Verlagsgruppe Beltz; 1969.

22. Arnold R, Krämer-Stürzl A, Siebert H. Dozentenleitfaden: Planung und Unterrichtsvorbereitung in Fortbildung und Erwachsenenbildung. Berlin: Cornelsen; 1999.

23. Harden RM. Learning outcomes and instructional objectives: is there a difference? Med Teach. 2002;24(2):151-155.

24. Harden RM. What is a spiral curriculum? Med Teach. 1999;21(2):141-143.

25. Bruner JS, ed. The process of education. 2nd ed. Cambridge, Mass.: Harvard University Press; 1960. 
26. Bloom BS, Engelhart MD, Messner R, Horn R, Füner E, eds. Taxonomie von Lernzielen im kognitiven Bereich. Weinheim: Verlagsgruppe Beltz; 1972.

27. Biggs J. Enhancing teaching through constructive alignment. Higher education. 1996;32(3):347-364.

28. Raupach T, Brown J, Anders S, Hasenfuss G, Harendza S. Summative assessments are more powerful drivers of student learning than resource intensive teaching formats. BMC Med.. 2013;11(1):61.

29. Fischer MR, Holzer M, Jünger J. Prüfungen an den medizinischen Fakultäten-Qualität, Verantwortung und Perspektiven. GMS Z Med Ausbild. 2010;27(5):Doc66.

30. Lernzielkatalog Zentrum Chirurgie. Frankfurt am Main: Fachbereich 16 Medizin der Universität Frankfurt am Main; 2013 Available from: https://www.uni-frankfurt.de/72583650/Lernzielkatalog_Chirurgie.pdf

31. Walcher F, Dreinhöfer K, Obertacke U, et al. Entwicklung des Lernzielkatalogs „Muskuloskelettale Erkrankungen, Verletzungen und traumatische Notfälle" für Orthopädie-Unfallchirurgie im Medizinstudium. Unfallchirurg. 2008;111(9):670-687.

32. Fischer MR, Bauer D, Karin Mohn N. Finally finished! National competence based catalogues of learning objectives for undergraduate medical education (NKLM) and dental education (NKLZ) ready for trial. GMS Z Med Ausbild. 2015;32(3).

33. Weidner K, Herrmann-Lingen C, Herzog W, et al. Lernziele der Psychosomatischen Medizin und Psychotherapie vor dem Hintergrund des Nationalen Kompetenzbasierten Lernzielkataloges Medizin (NKLM). [Learning objectives for psychosomatic medicine and psychotherapy in light of the National Competency-Based Catalogue of Learning Objectives for Medicine (NKLM)]. Z Psychosom Med Psychother. 2015;61(3):275-288.

34. Preisser A, Angerer P, Hildenbrand $\mathrm{S}$, Letzel $\mathrm{S}$. Neuer Lernzielkatalog für das Fach Arbeitsmedizin. Arbeitsmed Sozialmed Umweltmed. 2015;50(7):539-544.

35. Adili F, Kadmon M, König S, Walcher F. Professionalisierung der Lehre im chirurgischen Alltag. Chirurg. 2013;84(10):869-874.

36. Epstein RM, Hundert EM. Defining and assessing professional competence. JAMA. 2002;287(2):226-235. 
37. Weinert, FE. Concept of competence: A conceptual clarification. In Rychen DS \& Salganik LH, eds. Defining and selecting key competencies. Bern: Hogrefe \& Huber Publishers; 2001

38. Möltner A, Wagener S, Burkert M. Measuring competency-relevant knowledge in the competency-oriented student progress test. GMS J Med Educ. 2020;37(1).

39. Miller GE. The assessment of clinical skills/competence/performance. Acad Med. 1990;65(9):S63-67.

40. ten Cate O, Scheele F. Competency-based postgraduate training: can we bridge the gap between theory and clinical practice? Acad Med. 2007;82(6):542-547.

41. Kopp V, Möltner A, Fischer MR. Key-Feature-Probleme zum Prüfen von prozeduralem Wissen: Ein Praxisleitfaden. Gms Z Med Ausbild. 2006;23(3):2006-2023.

42. Faulkner D, Streefkerk C. Practice Ready Assessment for IMG Physicians. Medical Council of Canada - Annual General Meeting 2013 - Ottawa. 2013.

43. Schmidt HG, Moust J. Towards a taxonomy of problems used in problembased learning curricula. J Excellence Coll Teach. 2000;11(2/3):57-72.

44. McGaghie WC, Miller GE, Sajid AW, Telder TV. Competency-based curriculum development on medical education: an introduction. Public Health Pap. 1978;(68):11-91

45. Frank JR, Danoff D. The CanMEDS initiative: implementing an outcomesbased framework of physician competencies. Med Teach. 2007;29(7):642647.

46. Frank JR, Mungroo R, Ahmad Y, Wang M, De Rossi S, Horsley T. Toward a definition of competency-based education in medicine: a systematic review of published definitions. Med Teach. 2010;32(8):631-637.

47. Wissenschaftsrat (WR) Empfehlungen zur Weiterentwicklung des Medizinstudiums in Deutschland auf Grundlage einer Bestandsaufnahme der humanmedizinischen Modellstudiengänge. Dresden: Wissenschaftsrat; 2014. Available from: http://www.wissenschaftsrat.de/download/archiv/401714.pdf.

48. Harden RM. AMEE Guide No. 14: Outcome-based education: Part 1-An introduction to outcome-based education. Med Teach. 1999;21(1):7-14.

49. Koestner W, Otten W, Kaireit T, Wacker FK, Dettmer S. Competency-Based Teaching in Radiology - Implementation and Evaluation of Interactive 
Workstation-Based Learning to Apply NKLM-Based Content. RoFo. 2017;189(11):1076-1085.

50. Dath D, lobst W, Collaborators IC. The importance of faculty development in the transition to competency-based medical education. Med Teach. 2010;32(8):683-686.

51. Frank JR, Snell LS, Cate OT, et al. Competency-based medical education: theory to practice. Med Teach. 2010;32(8):638-645.

52. Hahn E, Fischer M. Nationaler Kompetenzbasierter Lernzielkatalog Medizin (NKLM) für Deutschland: Zusammenarbeit der Gesellschaft für Medizinische Ausbildung (GMA) und des Medizinischen Fakultätentages (MFT). GMS Z Med Ausbild. 2009;26(3):2009-2026.

53. Laan RF, Leunissen RR, Van Herwaarden C. The 2009 framework for undergraduate medical education in the Netherlands. GMS Z Med Ausbild. 2010;27(2):Doc35.

54. Neufeld VR, Maudsley RF, Pickering RJ, et al. Educating future physicians for Ontario. Acad Med. 1998;73(11):1133-1148.

55. Can M. Extract from the CanMEDS 2000 project societal needs working group report. Med Teach. 2000;22:549-554.

56. Fritze O, Griewatz J, Narciss E, et al. How much GK is in the NKLM? A comparison between the catalogues of exam-relevant topics (GK) and the German National Competence-based Learning Objectives Catalogue for Undergraduate Medical Education (NKLM). GMS J Med Educ. 2017;34(1):Doc9.

57. Kadmon M, Bender MJ, Adili F, et al. Kompetenzorientierung in der medizinischen Ausbildung. Chirurg. 2013;84(4):277-285.

58. Rademakers JJ, De Rooy N, Ten Cate OTJ. Senior medical students' appraisal of CanMEDS competencies. Med. Educ. 2007;41(10):990-994.

59. Ringsted C, Hansen TL, Davis D, Scherpbier A. Are some of the challenging aspects of the CanMEDS roles valid outside Canada? Med. Educ. 2006;40(8):807-815.

60. Stutsky BJ, Singer M, Renaud R. Determining the weighting and relative importance of CanMEDS roles and competencies. BMC Res Notes. 2012;5(1):1-7.

61. Jilg S, Möltner A, Berberat P, Fischer M, Breckwoldt J. Wie bewerten im Krankenhaus tätige Ärztinnen und Ärzte die Bedeutung der Rollendefinierenden Kompetenzen des CanMEDS-Modells und ihre Umsetzung für die Ausbildung im Praktischen Jahr. GMS Z Med Ausbild. 2015;32(3):10-15. 
62. Harden RM. AMEE Guide No. 21: Curriculum mapping: a tool for transparent and authentic teaching and learning. Med Teach. 2001;23(2):123-137.

63. Griewatz J, Wiechers S, Ben-Karacobanim H, Lammerding-Koeppel M. Medical teachers' perception of professional roles in the framework of the German National Competence-Based Learning Objectives for Undergraduate Medical Education (NKLM) -A multicenter study. Med Teach. 2016;38(11):1157-1165.

64. Lammerding-Koeppel M, Giesler M, Gornostayeva M, et al. Monitoring and analysis of the change process in curriculum mapping compared to the National Competency-based Learning Objective Catalogue for Undergraduate Medical Education (NKLM) at four medical faculties. Part II: Key factors for motivating the faculty during the process. GMS J Med Educ. 2017;34(1).

65. Sterz J, Adili F, Bender M, et al. Nationaler Kompetenzbasierter Lernzielkatalog Chirurgie - allgemeiner Teil mit fachbezogenen ärztlichen Handlungskompetenzen am Ende des Praktischen Jahres. [National Learning Objectives Catalogue in Surgery - General Part Defining Competences of Medical School Graduates in Surgery]. Zentralbl Chir. 2019;144(6):573-579.

66. Lammerding-Koeppel M, Giesler M, Gornostayeva M, et al. Monitoring and analysis of the change process in curriculum mapping compared to the National Competency-based Learning Objective Catalogue for Undergraduate Medical Education (NKLM) at four medical faculties. Part I: Conducive resources and structures. GMS J Med Educ. 2017;34(1):Doc7.

67. Medizinischer Fakultätentag (MFT) der Bundesrepublik Deutschland e. V. Faktenblatt Nationaler Kompetenzbasierter Lernzielkatalog Medizin und Zahnmedizin. (NKLM/NKLZ). https://medizinische-fakultaeten.de/wpcontent/uploads/2017/12/Faktenblatt-NKLM.pdf. Accessed December 29, 2020.

68. Medizinischer Fakultätentag (MFT) der Bundesrepublik Deutschland e. V. Angebote und Initiativen des Medizinischen Fakultätentages Der Weg zu kompetenzorientierter Lehre - ein Video zur Reform des Medizinstudiums. https://medizinische-fakultaeten.de/angebote/. Accessed December 29, 2020.

69. Harden R. The learning environment and the curriculum. Med Teach. 2001;23:335-336.

70. Davis $\mathrm{MH}$, Harden RM. Planning and implementing an undergraduate medical curriculum: the lessons learned. Med Teach. 2003;25(6):596-608. 
71. Zelenitsky S, Vercaigne L, Davies NM, Davis C, Renaud R, Kristjanson C. Using curriculum mapping to engage faculty members in the analysis of a pharmacy program. Am J Pharm Educ. 2014;78(7).

72. Hausman JJ. Mapping as an approach to curriculum planning. Curriculum Theory Net. 1974;4(2-3):192-198.

73. Willett TG. Current status of curriculum mapping in Canada and the UK. Med Educ. 2008;42(8):786-793.

74. Robley W, Whittle S, Murdoch-Eaton D. Mapping generic skills curricula: outcomes and discussion. J. Furth. High. Educ. 2005;29(4):321-330.

75. Smith SR. Planning, implementing and evaluating a competency-based curriculum. Med Teach. 1999;21:15-22.

76. Harendza S, Fischer MR, Fabry G. Quo vadis?--Medical education 2020 between politics and science. GMS J Med Educ. 2016;33(1):Doc12.

77. Fritze O, Lammerding-Koeppel M, Boeker M, et al. Boosting competenceorientation in undergraduate medical education-A web-based tool linking curricular mapping and visual analytics. Med Teach. 2019;41(4):422-432.

78. Lammerding-Koeppel M, Fritze O, Giesler M, et al. Benchmarking for research-related competencies-a curricular mapping approach at medical faculties in Germany. Med Teach. 2018;40(2):164-173.

79. Fritze O, Boecker M, Gornostayeva M, et al. Kompetenzorientiertes Curriculummapping im MERlin-Projekt: eine Online-Datenbank als Tool zur gezielten curricularen Weiterentwicklung. Jahrestagung der Gesellschaft für Medizinische Ausbildung (GMA). Hamburg, 25.-27.09.2014. Düsseldorf: German Medical Science GMS Publishing House, 2014. DocV232.

80. Fritze O, Giesler M, Gornostayeva M, et al. Kompetenzorientierte Lehre gezielte Entwicklung longitudinaler Kompetenzstränge. Gemeinsame Jahrestagung der Gesellschaft für Medizinische Ausbildung (GMA) und des Arbeitskreises zur Weiterentwicklung der Lehre in der Zahnmedizin (AKWLZ); 30.09.-03.10.2015; Leipzig. Düsseldorf: German Medical Science GMS Publishing House; 2015. pp. DocP3-Do044.

81. Bland CJ, Starnaman S, Wersal L, Moorhead-Rosenberg L, Zonia S, Henry R. Curricular change in medical schools: how to succeed. Acad Med. 2000;75(6):575-594.

82. Sterz J, Hoefer SH, Janko M, et al. Do they teach what they need to? An analysis of the impact of curriculum mapping on the learning objectives taught in a lecture series in surgery. Med Teach. 2019;41(4):417-421. 
83. Gerhardt-Szép S, Brandt S, Hoefer SH, et al. Interdisziplinäre Curriculumskartierung der Frankfurter zahnmedizinischen Ausbildung anlehnend an den NKLZ. Dtsch Zahnarztl Z. 2016;71(2).

84. Rüsseler M, Weber R, Braunbeck A, Flaig W, Marzi I, Walcher F. Training praktischer Fertigkeiten in der Chirurgie - Ein Ausbildungskonzept für Studierende. Zentralb/ Chir. 2010;135(3):249-256.

85. Ruesseler M, Schill A, Stibane T, et al. „Praktische klinische Kompetenz “ ein Verbundprojekt zur Verbesserung der chirurgischen Lehre. ["Practical clinical competence" - a joint programme to improve training in surgery]. Zentralb/ Chir. 2013;138(6):663-668.

86. Hessisches Ministerium für Soziales und Integration. Versorgungsatlas Hessen 2017: Leistungsreport der hessischen Plankrankenhäuser 2017. https://soziales.hessen.de/sites/default/files/media/hsm/versorgungsatlas_h essen_2017_leistungsreport_der_hessischen_plankrankenhaeuser.pdf. Accessed December 29, 2020.

87. Bugaj TJ, Schmid C, Koechel A, et al. Shedding light into the black box: A prospective longitudinal study identifying the CanMEDS roles of final year medical students' on-ward activities. Med Teach. 2017;39(8):883-890.

88. Turner SR, White JS, Poth C, Rogers WT. Learning the CanMEDS roles in a near-peer shadowing program: a mixed methods randomized control trial. Med Teach. 2012;34(11):888-892.

89. Hassan IS, Kuriry H, Ansari LA, et al. Competency-structured case discussion in the morning meeting: enhancing CanMEDS integration in daily practice. Adv Med Educ Pract. 2015;6:353-358.

90. Ponton-Carss AC, Donnon T, Kortbeek JB. Two for one: surgical skills and CanMEDS roles--a combined course for surgical residents. J Surg Educ. 2014;71(3):419-425.

91. Huber-Lang M, Palmer A, Grab C, Boeckers A, Boeckers TM, Oechsner W. Visions and reality: the idea of competence-oriented assessment for German medical students is not yet realised in licensing examinations. GMS J Med Educ. 2017;34(2).

92. Kassam A, Cowan M, Donnon T. An objective structured clinical exam to measure intrinsic CanMEDS roles. Med Educ Online. 2016;21:31085.

93. Dwyer T, Takahashi SG, Hynes MK, et al. How to assess communication, professionalism, collaboration and the other intrinsic CanMEDS roles in orthopedic residents: use of an objective structured clinical examination (OSCE). Can J Surg. 2014;57(4):230. 
94. Fromme HB, Karani R, Downing SM. Direct observation in medical education: a review of the literature and evidence for validity. Mt Sinai $\mathrm{J}$ Med. 2009;76(4):365-371.

95. Norcini J, Burch V. Workplace-based assessment as an educational tool: AMEE Guide No. 31. Med Teach. 2007;29(9-10):855-871.

96. Schelling J, Sanftenberg L, Holzer M. Erfahrungen mit arbeitsplatzbasiertem Prüfen im Blockpraktikum. Z Allg Med. 2017;93(4).

97. Birch DW, Mavis B. A needs assessment study of undergraduate surgical education. Can J Surg. 2006;49(5):335.

98. Federkeil G. CHE Alumni-Ranking Medizin: Ergebnisse einer vergleichenden Absolventenbefragung Humanmedizin des Centrums für Hochschulentwicklung. Arbeitspapier Nr. 57. Gütersloh: Centrum für Hochschulentwicklung; 2004.

99. Prince KJ, Boshuizen HP, Van Der Vleuten CP, Scherpbier AJ. Students' opinions about their preparation for clinical practice. Med. Educ. 2005;39(7):704-712.

100. Remmen R, Derese A, Scherpbier A, et al. Can medical schools rely on clerkships to train students in basic clinical skills? Med. Educ. 1999;33(8):600-605.

101. Stibane T, Schönbauer A, Jerrentrup A, Pressel T, Baum E, Bösner S. Systematischer praktischer Unterricht führt zu mehr praktischer Kompetenz. Z Allg Med. 2012;88(4):184-191.

102. Rüsseler M, Schill A, Kalozoumi-Paisi P, et al. Lehre im Fokus-Wie beurteilen Studierende ihre praktisch-klinische Ausbildung in der Chirurgie? Zentralb/ Chir. 2017;142(01):46-53.

103. Bornemann S, Stosch C. Akzeptanz und Nutzen des Zertifikats „Famulaturreife" bei Kliniken in Köln und Umgebung. In: Jahrestagung der Gesellschaft für Medizinische Ausbildung (GMA). Hamburg, 25.-27.09.2014. Düsseldorf: German Medical Science GMS Publishing House; 2014. DocP171

104. Ochsmann EB, Zier U, Drexler H, Schmid K. Well prepared for work? Junior doctors' self-assessment after medical education. BMC Med Educ. 2011 Nov 24;11:99.

105. Brennan N, Corrigan $\mathrm{O}$, Allard J, et al. The transition from medical student to junior doctor: today's experiences of Tomorrow's Doctors. Medical education. 2010;44(5):449-458. 
106. Statistisches Bundeamt. Tabelle: Studierende nach Semester, Nationalität, Geschlecht und Studienfach. https://wwwgenesis.destatis.de/genesis/online?sequenz=tabelleErgebnis\&selectionnam e=21311-0003\#abreadcrumb. Accessed November 17, 2020.

107. Statistisches Bundesamt. Ärzte in Deutschland. Statista Dossier zum Thema deutsche Ärzteschaft.

https://de.statista.com/statistik/studie/id/6488/dokument/aerzte-statistadossier. Accessed November 17, 2020.

108. Gedrose B, Wonneberger C, Jünger J, et al. Haben Frauen am Ende des Medizinstudiums andere Vorstellungen über Berufstätigkeit und Arbeitszeit als ihre männlichen Kollegen? [Do female medical graduates have different views on professional work and workload compared to their male colleagues? Results of a multicenter postal survey in Germany]. Dtsch Med Wochenschr. 2012 Jun;137(23):1242-7.

109. Risberg G, Johansson EE, Westman G, Hamberg K. Gender in medicine-an issue for women only? A survey of physician teachers' gender attitudes. Int $J$ Equity Health. 2003;2(1):10.

110. Ro HK, Knight DB. Gender differences in learning outcomes from the college experiences of engineering students. J. Eng. Educ. 2016;105(3):478-507.

111. Kalender ZY, Marshman E, Nokes-Malach T, Schunn C, Singh C. Large gender differences in physics self-efficacy at equal performance levels: $\mathrm{A}$ warning sign. In Physics Education Research Conference 2018, PER Conference, Washington, DC, August 1-2 2018.

112. T. J. Nokes-Malach, Z. Y. Kalender, E. Marshman, C. Schunn, and C. Singh. Prior preparation and motivational characteristics mediate relations between gender and learning outcomes in introductory physics. In Physics Education Research Conference 2018, PER Conference, Washington, DC, August 1-2 2018.

113. Zimmerman BJ. Self-efficacy: An essential motive to learn. Contemp Educ Psychol. 2000;25(1):82-91.

114. Seymour E, Hewitt NM. Talking about leaving. Westview Press, Boulder, CO; 1997.

115. Lindlohr C, Pape- Köhler C, Toaspern J, Lefering R, Heiss MM. Sind Frauen die besseren Operateure? Der Einfluss des Geschlechts auf den chirurgischen Lernerfolg am Pelvitrainer nach unterschiedlichen Trainingsmethoden. Z Gastroenterol. 2011;49(08):V56. 
116. Jankowski J, Crombie I, Block R, Mayet J, McLay J, Struthers A. Selfassessment of medical knowledge: do physicians overestimate or underestimate? J R Coll Physicians Lond. 1991;25(4):306.

117. Borracci RA, Alvarez-Gallesio JM, Ciambrone G, Mezzadri NA. Selfestimation of surgical skills and competencies based on the learning curve theory in medical residents and fellows. Cir Cir. 2019;87(4):416-422.

118. Blanch-Hartigan D. Medical students' self-assessment of performance: results from three meta-analyses. Patient Educ Couns. 2011;84(1):3-9.

119. Czeskleba A, Holzhausen $\mathrm{Y}$, Peters H. Patient safety during final-year clerkships: A qualitative study of possible error sources and of the potential of Entrustable Professional Activities. GMS J Med Educ. 2019;36(2).

120. Berberat PO, Rotthoff T, Baerwald C, et al. Entrustable Professional Activities in final year undergraduate medical training-advancement of the final year training logbook in Germany. GMS J Med Educ. 2019;36(6).

121. Sterz J, Britz V, Kollewe T. Der Chirurg im Spagat - Lehre im klinischen Alltag. 2016. In: Deutsche Gesellschaft für Chirurgie. 133. Kongress der Deutschen Gesellschaft für Chirurgie. Berlin, 26.-29.04.2016. Düsseldorf: German Medical Science GMS Publishing House; 2016. Doc16dgch473

122. König S, Stieger P, Sippel S, et al. Train-the-Trainer: Professionalisierung der Lehre im klinischen Altag-Selbsteinschätzung Lehrender zur didaktischen Kompetenz und den Rahmenbedingungen des Unterrichts. Zentralb/ Chir. 2019;144(06):551-559 


\section{Abbildungsverzeichnis}

Abb. 1 Modifizierte Pyramide nach Miller (Miller 1990) mit geeigneten

Prüfungsformaten

Abb. 2 Vergleich der angewendeten Kompetenzebenen bei Miller, im NKLM und im SCLO

Abb. 3 Aufbau der Abschnitte 1-3 des NKLM 21

Abb. 4 Auszug aus Kapitel 12 des NKLM 22

Abb. 5 Layout Onlinebasierter Fragekatalog Survey Monkey 34

Abb. 6 Geschlechterverteilung der Studienteilnehmenden 40

Abb. 7 Verteilung der besuchten Fachrichtungen 41

Abb. 8 Verteilung der Studierenden auf die einzelnen (Lehr-)Krankenhäuser 42

Abb. 9 Verteilung der im Blockpraktikum erreichten Lernziele 42

Abb. 10 Insgesamt erreichte Lernziele der Abschnitte 1, 2 und 3 des NKLM 43

Abb. 11 Insgesamt erreichte Lernziele der Kapitel 5-11 in Abschnitt 1

Abb. 12 Implizit vermittelte Lernziele in Abschnitt 1 des NKLM 45

Abb. 13 Erreichte Lernziele der Kapitel 12 - 19 in Abschnitt 2

Abb. 14 Erreichte Lernziele der Kapitel 20 und 21 in Abschnitt 3

Abb. 15 Erreichte Lernziele der Unterkapitel des Kapitel 21 zu Primären

Organsystemen 48

Abb. 16 Insgesamt erreichte Lernziele nach Geschlecht 49

Abb. 17 Erreichte Lernziele in Abschnitt 1 nach Geschlecht 50

Abb. 18 Erreichte Lernziele in Abschnitt 2 nach Geschlecht 51

Abb. 19 Erreichte Lernziele des Abschnitt 2 (speziell Kapitel 14 a - c) nach Geschlecht 52

Abb. 20 Erreichte Lernziele des Abschnitt 3 nach Geschlecht 53

Abb. 21 Insgesamt erreichte Lernziele nach Fachabteilungen 54

Abb. 22 Erreichte Lernziele des Abschnitt 1 nach Fachabteilungen 55

Abb. 23 Erreichte Lernziele des Abschnitt 2 (Kap. 12, 13, 15 - 19) nach

Fachabteilungen $\quad 57$

Abb. 24 Erreichte Lernziele der Kapitel 14 a - c nach Fachabteilungen 58

Abb. 25 Erreichte Lernziele des Abschnitt 3 nach Fachabteilungen 60 
Abb. 26 Erreichte Lernziele ausgewählter Unterkapiteln des Kapitel 21 zu Primären Organsystemen nach Fachabteilungen

Abb. 27 Insgesamt erreichte Lernziele nach (Lehr-) Krankenhäusern 63

Abb. 28 Erreichte Lernziele des Abschnitt 1 nach (Lehr-)Krankenhäusern

Abb. 29 Erreichte Lernziele des Abschnitt 2 (Kapitel 12, 13, 15 - 19) nach (Lehr-) Krankenhäusern

Abb. 30 Erreichte Lernziele des Abschnitt 2 (Kapitel 14 a - c) nach (Lehr-)

Krankenhäusern

Abb. 31 Erreichte Lernziele des Abschnitt 3 nach (Lehr-)Krankenhäusern 


\section{Tabellenverzeichnis}

Tab. 1 Auflistung der Kapitel des Abschnitts 1

Tab. 2 Auflistung der Kapitel des Abschnitt 2

Tab. 3 Auflistung der Kapitel des Abschnitt 3

Tab. 4 Auflistung der Lehrkrankenhäuser und deren Anzahl chirurgischer $\begin{array}{ll}\text { Bettenplätze } & 37\end{array}$

Tab. 5 Erreichte Lernziele des Abschnitts 1 des NKLM 44

Tab. 6 Implizit vermittelte Lernziele des Abschnitt 1

Tab. 7 Erreichte Lernziele des Abschnitts 2 des NKLM 46

Tab. 8 Erreichte Lernziele der Unterkapitel des Kapitel 21 zu Primären Organsystemen 48

Tab. 9 Erreichte Lernziele des Abschnitt 1 nach Geschlecht 50

Tab. 10 Erreichte Lernziele des Abschnitt 2 nach Geschlecht 51

Tab. 11 Insgesamt erreichte Lernziele nach Fachrichtungen 54

Tab. 12 Erreichte Lernziele des Abschnitt 1 nach Fachrichtungen 55

Tab. 13 Erreichte Lernziele des Abschnitt 2 nach Fachrichtungen 58

Tab. 14 Erreichte Lernziele des Abschnitt 3 nach Fachrichtungen 60

Tab. 15 Erreichte Lernziele ausgewählter Unterkapitel des Kapitel 21 nach Fachrichtungen 62

Tab. 16 Insgesamt erreichte Lernziele nach (Lehr-)Krankenhäusern 63

Tab. 17 Erreichte Lernziele des Abschnitt 1 nach (Lehr-)Krankenhäusern $\quad 64$

Tab. 18 Erreichte Lernziele des Abschnitt 2 nach (Lehr-)Krankenhäusern $\quad 67$

Tab. 19 Erreichte Lernziele des Abschnitt 3 nach (Lehr-)Krankenhäusern 69 


\section{Anhang}

10.1 Inhaltsverzeichnis NKLM mit Anzahl der Lernziele 102

10.2 Testatheft Blockpraktikum Chirurgie 104

\subsection{Inhaltsverzeichnis NKLM mit Anzahl der Lernziele}

\begin{tabular}{|c|c|c|}
\hline Kapitel & Überschrift & Anzahl Lernziele \\
\hline \multicolumn{2}{|l|}{ GESAMT } & 2105 \\
\hline \multicolumn{2}{|c|}{ Abschnitt 1 "Rollen der Ärztin / des Arztes" } & 189 \\
\hline Kapitel 5 & Der Arzt als medizinischer Experte & 24 \\
\hline Kapitel 6 & Der Arzt als Gelehrter & 32 \\
\hline Kapitel 7 & Der Arzt als Kommunikator & 6 \\
\hline Kapitel 8 & Der Arzt als Mitglied eines Teams & 24 \\
\hline Kapitel 9 & Der Arzt als Gesundheitsberater/-fürsprecher & 18 \\
\hline Kapitel 10 & Der Arzt als Verantwortungsträger und Manager & 37 \\
\hline Kapitel 11 & Der Arzt als professionell Handelnder & 48 \\
\hline \multicolumn{3}{|c|}{$\begin{array}{l}\text { Abschnitt } 2 \text { "Medizinisches Wissen, klinische Fähigkeiten und professionelle } \\
\text { Haltungen" }\end{array}$} \\
\hline Kapitel 12 & Prinzipien normaler Struktur und Funktion & 411 \\
\hline Kapitel 13 & Prinzipien der Pathogenese und Pathomechanismen & 150 \\
\hline Kapitel 14 a & Medizinisch-wissenschaftliche Fertigkeiten & 38 \\
\hline Kapitel 14 b & Klinisch praktische Fertigkeiten & 92 \\
\hline Kapitel 14 c & Ärztliche Gesprächsführung & 116 \\
\hline Kapitel 15 & Diagnostische Verfahren & 91 \\
\hline Kapitel 16 & Therapeutische Prinzipien & 263 \\
\hline Kapitel 17 & Notfallmaßnahmen & 36 \\
\hline Kapitel 18 & Ethik, Geschichte und Recht der Medizin & 70 \\
\hline Kapitel 19 & Gesundheitsförderung und Prävention & 84 \\
\hline \multicolumn{2}{|c|}{ Abschnitt 3 "Patientenzentrierte Gesundheitsversorgung" } & 565 \\
\hline Kapitel 20 & Anlässe für ärztliche Konsultation & 124 \\
\hline \multirow[t]{8}{*}{ Kapitel 21} & $\begin{array}{l}\text { Erkrankungsbezogene Prävention, Diagnostik, Therapie, Versorgungs- } \\
\text { und Notfallmaßnahmen } \\
\text { Unterkapitel }\end{array}$ & 441 \\
\hline & Primäres Organsystem: Kardiovaskulär & 29 \\
\hline & Primäres Organsystem: Muskuloskelettal und Weichgewebe & 48 \\
\hline & Primäres Organsystem: Hormone und Stoffwechsel & 30 \\
\hline & Primäres Organsystem: Respiratorisches System & 34 \\
\hline & Primäres Organsystem: Blut und Immunologie & 21 \\
\hline & Primäres Organsystem: Urogenitales System & 44 \\
\hline & Primäres Organsystem: Verdauungssystem & 48 \\
\hline
\end{tabular}


Primäres Organsystem: Haut, Hautanhang, Schleimhaut

Primäres Organsystem: Sinnessysteme

Primäres Organsystem: Nervensystem und Psyche 


\subsection{Testatheft Blockpraktikum Chirurgie}
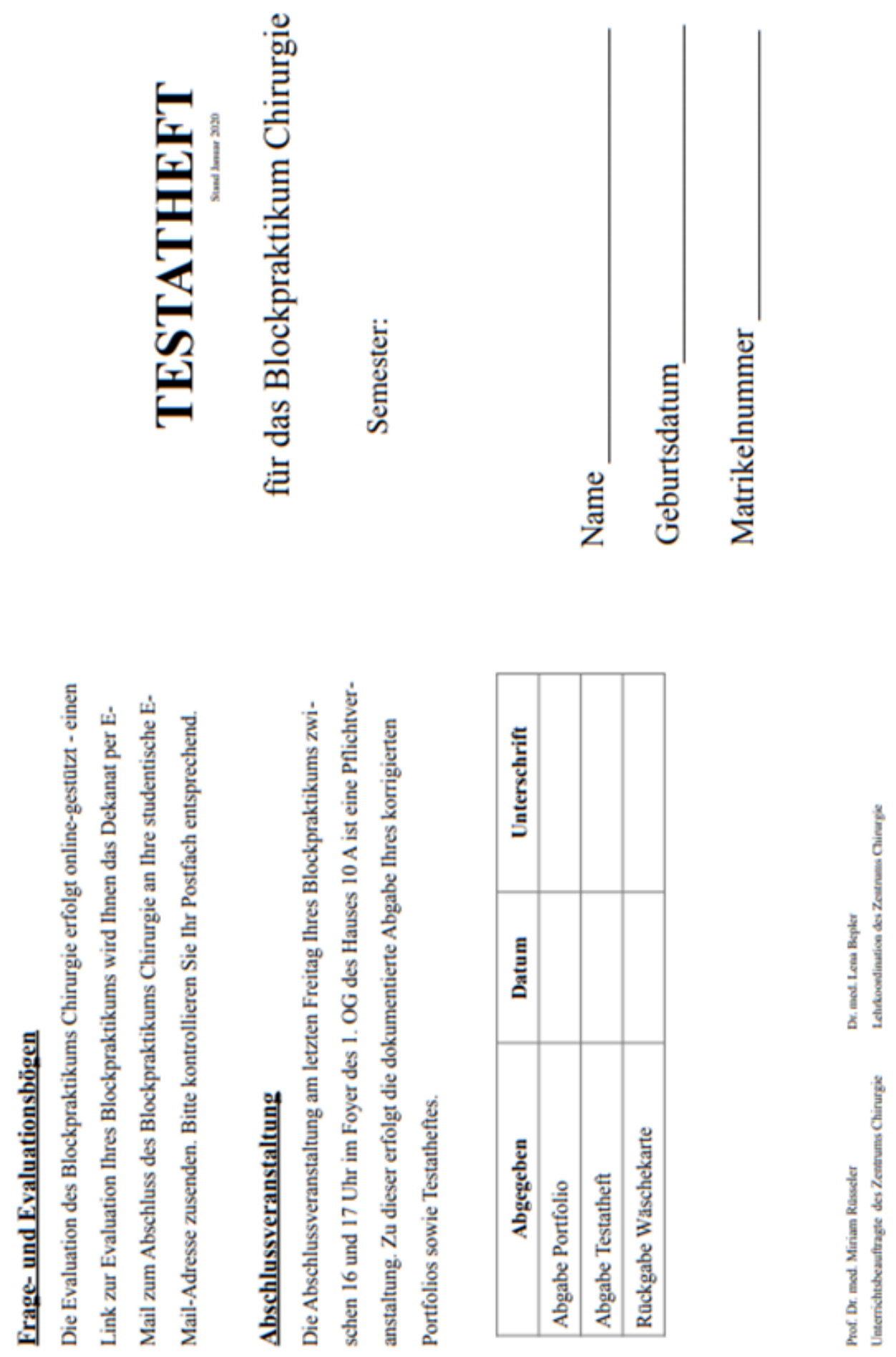

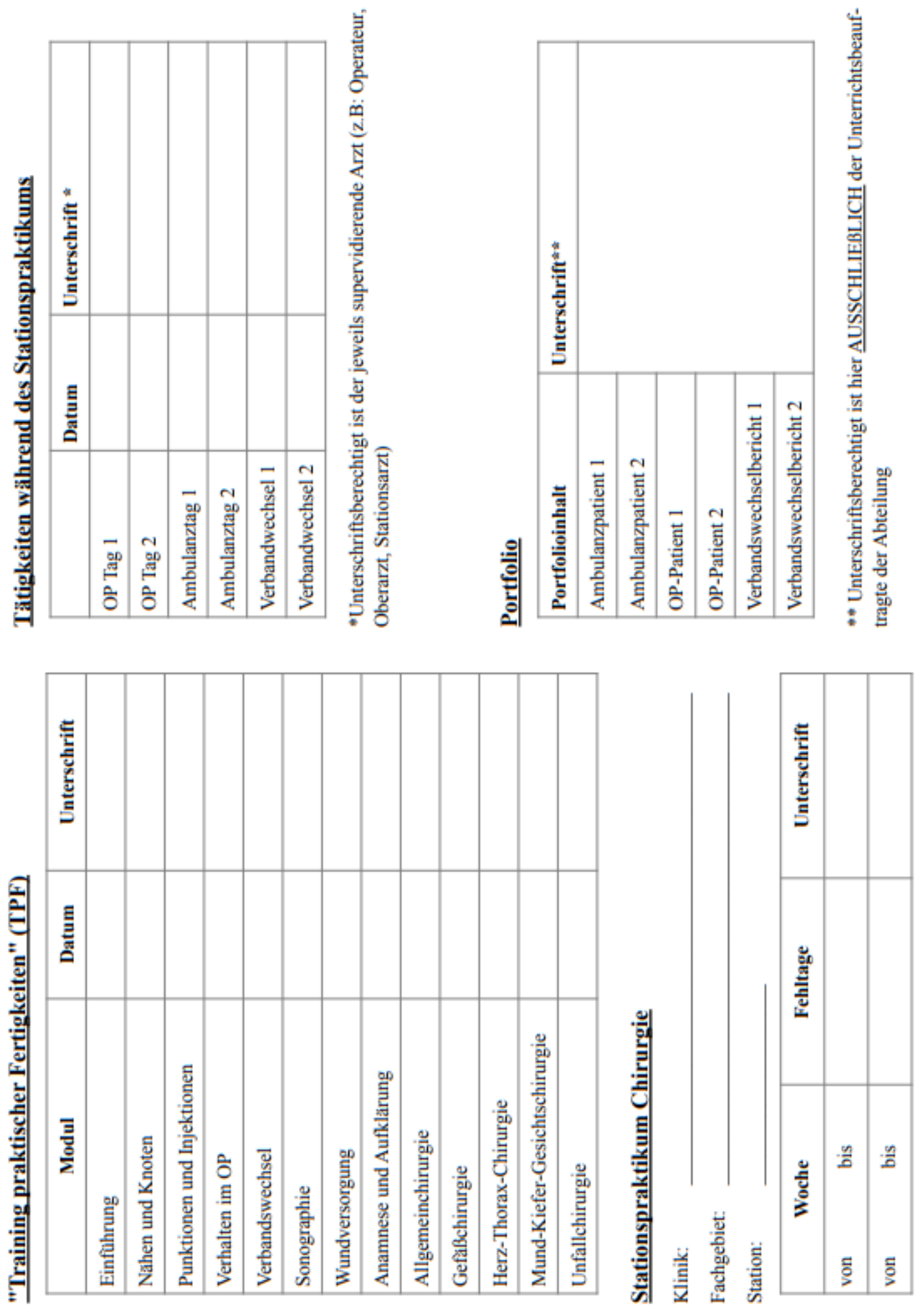
11. Danksagung 
12. Curriculum vitae 


\section{Vorträge}

A. - M. Weber, J. Sterz, M. - C. Stefanescu, H. Sterz, M. Rüsseler: „Welche Kompetenzen erwerben Studierende im Blockpraktikum Chirurgie? Mapping des Blockpraktikums mit dem NKLM." Jahrestagung der Gesellschaft für Medizinische Ausbildung (GMA), 27.09.2019, Frankfurt (Deutschland)

A.-M. Weber: „Welche Kompetenzen erwerben Studierende im Blockpraktikum Chirurgie? Mapping des Blockpraktikums mit dem NKLM."136. Kongress Deutsche Gesellschaft für Chirurgie (DCK), 28.03.2019, München (Deutschland) 


\section{Schriftliche Erklärung}

Ich erkläre ehrenwörtlich, dass ich die dem Fachbereich Medizin der Johann Wolfgang Goethe-Universität Frankfurt am Main zur Promotionsprüfung eingereichte Dissertation mit dem Titel

Welche Kompetenzen erwerben Studierende im Blockpraktikum Chirurgie? Curriculum Mapping mit dem NKLM

in der Klinik für Unfall-, Hand- und Wiederherstellungschirurgie der Universitätsklinik Frankfurt unter Betreuung und Anleitung von Prof. Dr. Miriam Rüsseler mit Unterstützung durch Dr. Jasmina Sterz ohne sonstige Hilfe selbst durchgeführt und bei der Abfassung der Arbeit keine anderen als die in der Dissertation angeführten Hilfsmittel benutzt habe. Darüber hinaus versichere ich, nicht die Hilfe einer kommerziellen Promotionsvermittlung in Anspruch genommen zu haben.

Ich habe bisher an keiner in- oder ausländischen Universität ein Gesuch um Zulassung zur Promotion eingereicht. Die vorliegende Arbeit wurde bisher nicht als Dissertation eingereicht.

Ergebnisse der Dissertation wurden bisher nicht veröffentlicht.

Frankfurt, 04.01.2021

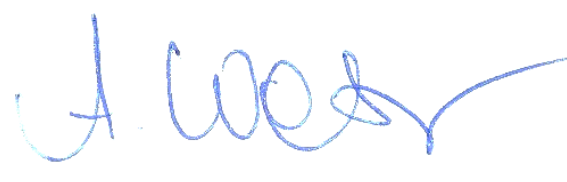

(Ort, Datum)

(Unterschrift) 